Portland State University

PDXScholar

$5-29-2020$

\title{
Where's the Learning in On-Campus Student Employment? An Inquiry into the Experiences of Undergraduate Student Employees
}

Patricia Ann Dorman

Portland State University

Follow this and additional works at: https://pdxscholar.library.pdx.edu/open_access_etds

Part of the Educational Leadership Commons

Let us know how access to this document benefits you.

\section{Recommended Citation}

Dorman, Patricia Ann, "Where's the Learning in On-Campus Student Employment? An Inquiry into the Experiences of Undergraduate Student Employees" (2020). Dissertations and Theses. Paper 5473. https://doi.org/10.15760/etd.7345

This Dissertation is brought to you for free and open access. It has been accepted for inclusion in Dissertations and Theses by an authorized administrator of PDXScholar. Please contact us if we can make this document more accessible: pdxscholar@pdx.edu. 
Where's the Learning in On-Campus Student Employment?

An Inquiry into the Experiences of Undergraduate Student Employees

\author{
by \\ Patricia Ann Dorman
}

A dissertation submitted in partial fulfillment of the requirements for the degree of

Doctor of Education

in

Educational Leadership: Postsecondary Education

Dissertation Committee:

Christine Cress, Chair

Karen Haley

Andy Job

Craig Shinn

Portland State University

2020 


\begin{abstract}
Student employees spend a significant amount of time at work. Prior research has primarily focused on the deleterious academic impacts on students, with little consideration of how student employment could be effectively framed as an educationally purposeful activity. This qualitative study explored how individual, social, and organizational factors influence learning while working on campus. In-depth, semistructured interviews were conducted with undergraduate student employees who work within student affairs units. The findings indicate that on-campus employment is a site for learning; particularly competency development in communication, professionalism, problem solving, and leadership skills. Interdependent personal, interpersonal, and environmental factors of campus employment were identified as positively influencing these student learning outcomes. Further, four mediating factors were identified which intensified learning when experienced by student employees: work/life balance, applied learning, sense of belonging, and self-efficacy. A Campus Employment Reciprocal Learning Model is proposed to both explain the conditions that facilitate learning experiences in campus workplaces and offer a framework for practitioners to provide campus employment experiences that facilitate learning while working for undergraduate student employees.
\end{abstract}




\section{Acknowledgements}

It truly took a village to bring me to the finish line of this doctoral experience. I want to first thank my husband, Daryll, who gave me endless encouragement and carried the heavy load of our domestic lives these past three years without ever once complaining. I am thankful to my family for their unwavering faith in my ability to accomplish this feat. Thank you to Angie and Chris who let me stay in your home every weekend I had classes. I appreciate all the feedback and advocacy provided by my chair, Dr. Christine Cress, who recognized the value of this research and pushed me to present it in the best possible light. I thoroughly enjoyed engaging in related critical discourse with all the members of my committee, Dr. Karen Haley, Dr. Andy Job, and Dr. Craig Shinn, and I am thankful for their support throughout this process. I am thankful to my cohort-mates for long drives to Portland, coffee shop workdays, weekends at the coast, text message check-ins, and getting through this together. I also want to thank Laurel Dean, who read through multiple iterations of this paper and offered her editing expertise. I am forever grateful to the incredible team I work with in PE \& Rec, who helped me balance work and school, listened to me to bounce ideas and run through presentations, and (occasionally) let me sit in their office and cry. I also want to acknowledge the Student Employment Enhancement committee, who passionately and tirelessly advocate for meaningful campus employment experiences. I chose this research topic because I have seen the positive impact and transformational power of student employment. To the hundreds of student employees I have had the pleasure of working with over my career and the many more still to come, this research is for you. 
Table of Contents

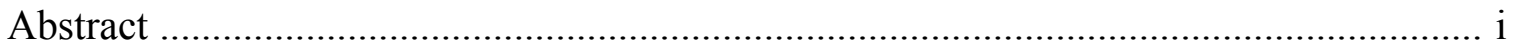

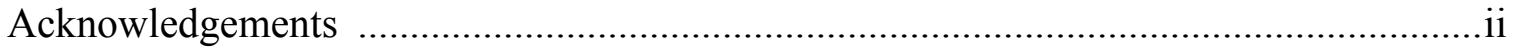

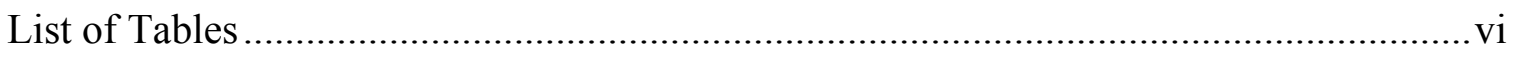

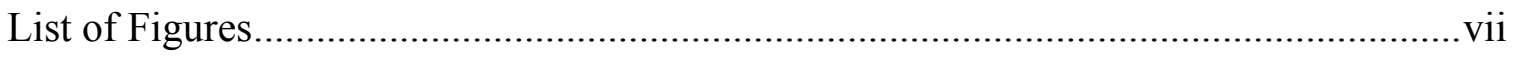

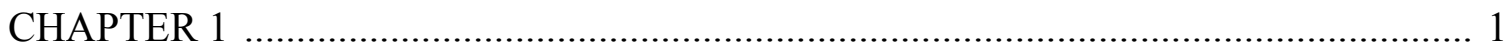

Background of the Problem .............................................................................................. 2

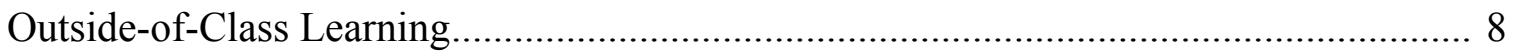

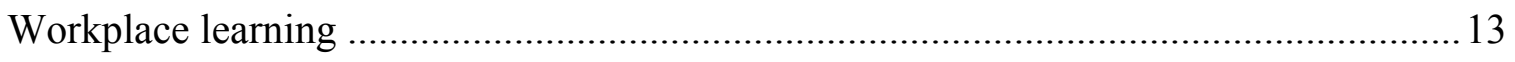

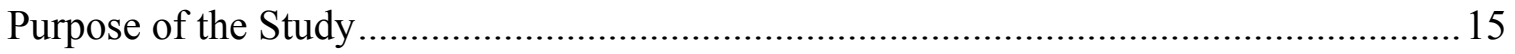

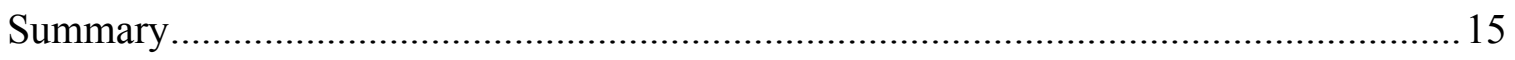

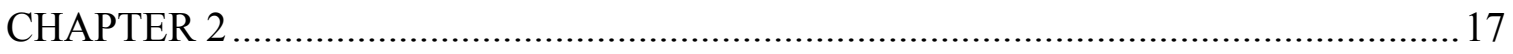

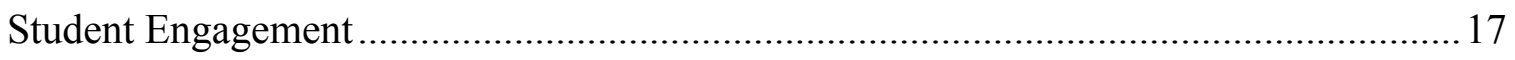

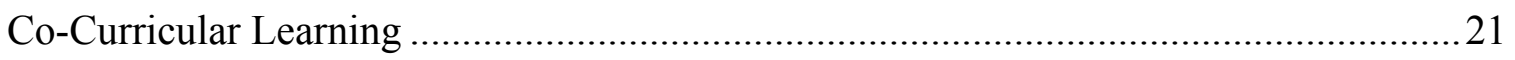

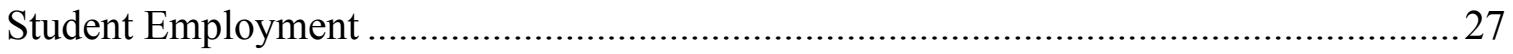

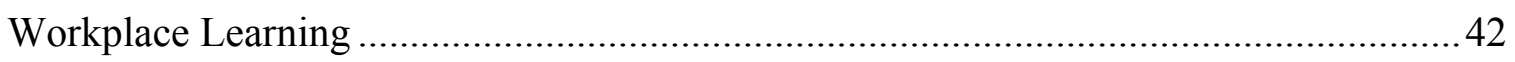

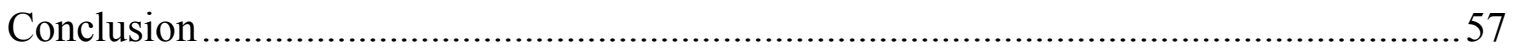

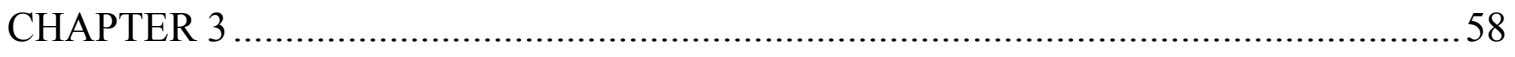

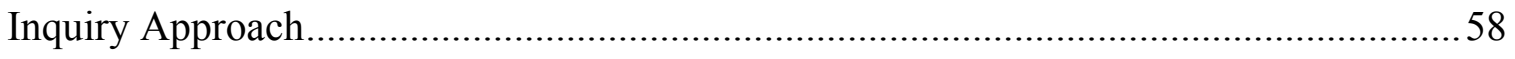

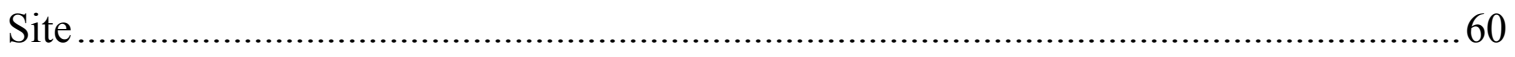

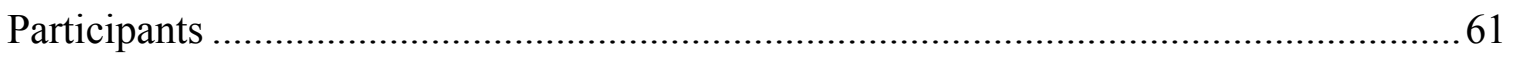

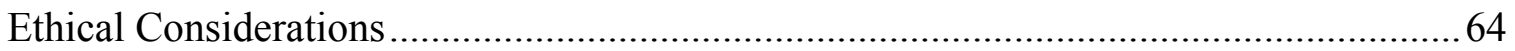




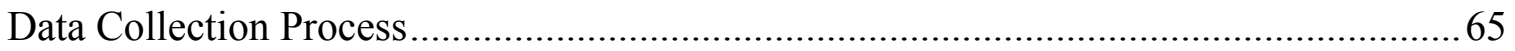

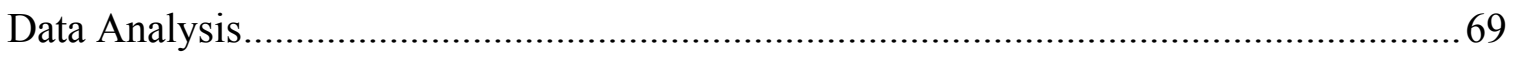

Researcher Positionality ……………………………..............................................

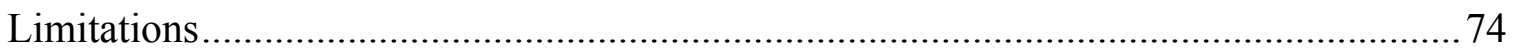

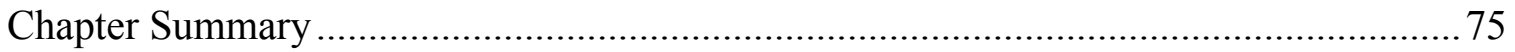

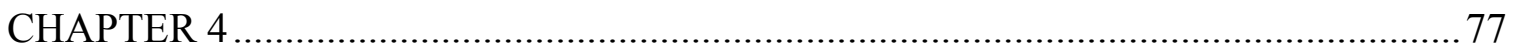

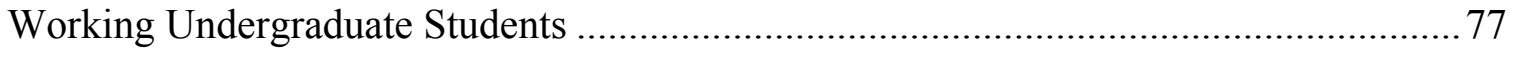

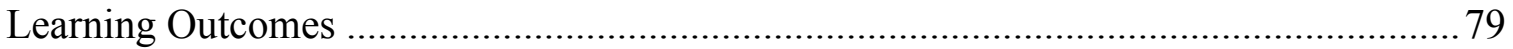

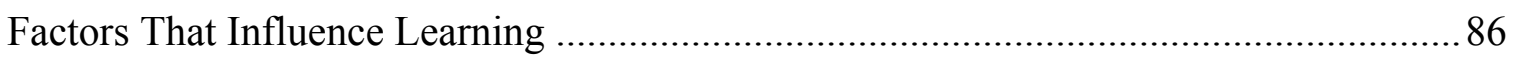

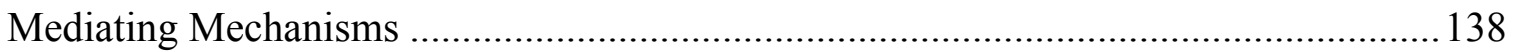

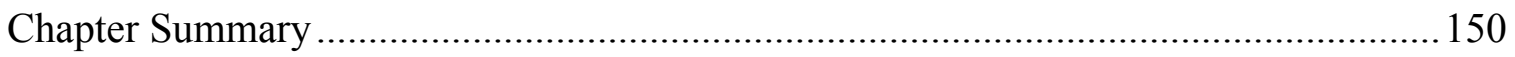

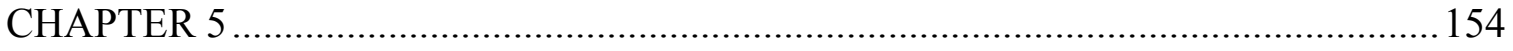

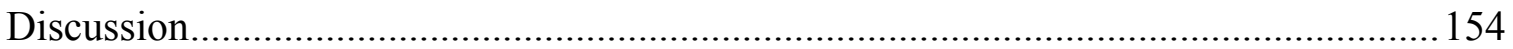

Campus Employment Reciprocal Learning Model ....................................................... 156

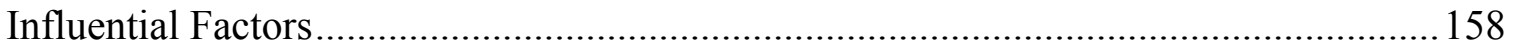

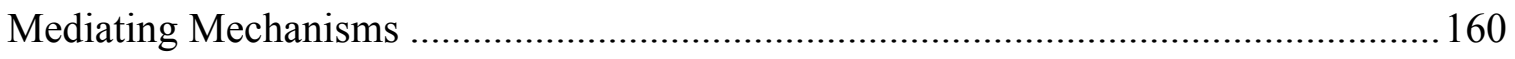

Academic and Professional Identity Development …………………........................... 163

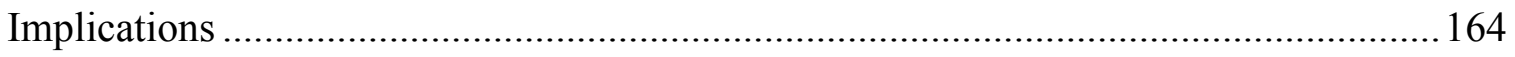

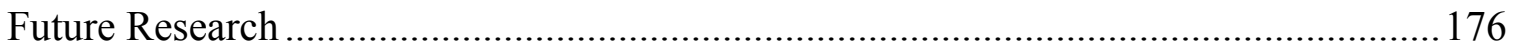

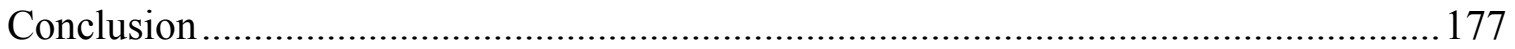

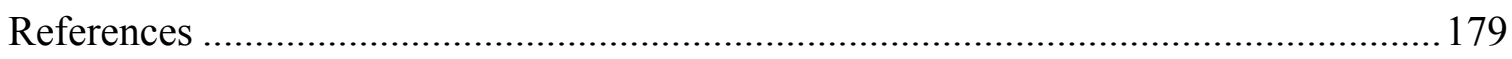

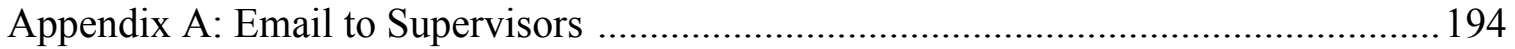


Appendix B: Email to Potential Participants

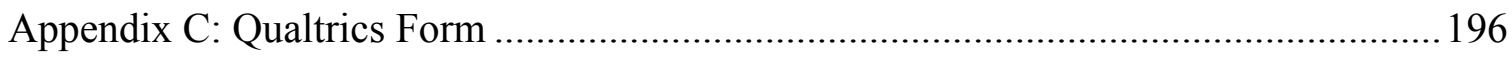

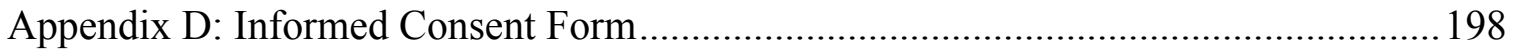

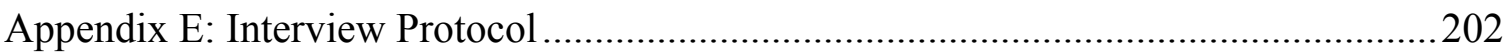




\section{List of Tables}

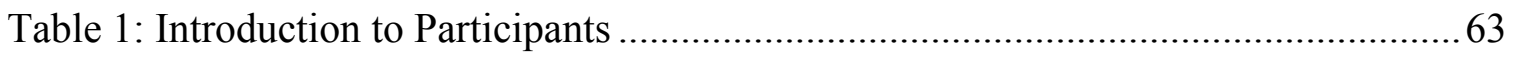

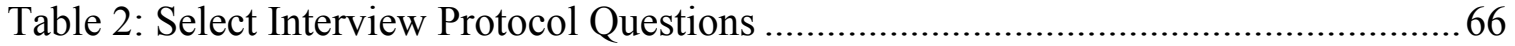

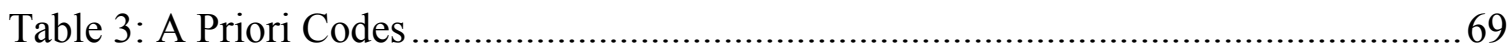

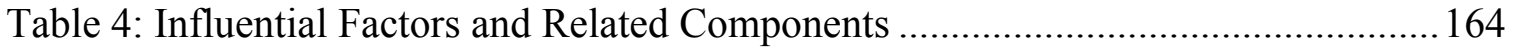




\section{List of Figures}

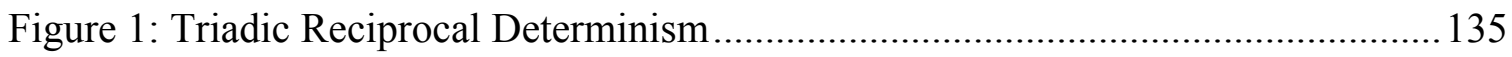

Figure 2: Campus Employment Reciprocal Learning Model...................................... 157 


\section{CHAPTER 1}

Students have worked while attending college throughout the entire history of higher education (Tuttle, McKinney, \& Rago, 2005); however, contemporary American undergraduate students are working at higher rates today than at any other point. According to Tuttle et al. (2005), 40\% of American college students worked while attending college in 1961 and that number steadily grew to $80 \%$ in 2000 . In the most recently available report from the National Center for Education Statistics, $79 \%$ of undergraduate college students reported working at least one hour per week (Perozzi, 2009). McClellan, Creager, \& Savoca (2018) aptly describe working in college as a "defining characteristic of today's student experience" that is surpassed only by attending classes as the most universal college student experience (p. 2).

Although there are a number of reasons that students seek employment during college, financial need tends to play a big role. Many students report their primary motivation for working during college is to cover tuition, fees, and basic living expenses (Cheng \& Alcántara, 2007; King, 2006; Tuttle et al., 2005). Burnside et al. (2019) refer to this as the working student dilemma. "The time, energy, and resources required to succeed as an employee and as a student can present working students with tough tradeoffs and decisions on how to handle these competing priorities" (p. 6). Balancing the increasing financial obligations of college and the limited options for financial aid accounts for a large part of the growth in full-time undergraduate students working while attending college. The cost of higher education continues to rise, and as students exhaust financial aid options, many turn to work to cover the gap in their financial needs. 
With so many students working while attending college, it is important to understand how the work experience affects college student learning, development, and growth; yet research on the topic has largely perpetuated a deficit mindset toward student employment which assumes that working and academic learning are necessarily in competition, rather than working in tandem toward achieving the goals of higher education. Further, research on the effects of student employment on academic outcomes have produced contradictory and inconclusive results (Pascarella \& Terenzini, 2005;

Pike, Kuh, \& Massa-McKinley, 2008). The opportunity to influence student employment conditions in off-campus positions is limited; however, the on-campus work environment which is directed by the efforts of institutional members, henceforth referred to as campus employment, is ripe with opportunity to positively influence student learning and development.

Chapter 1 will start by introducing the problem through detailing various dimensions of student employment, including who works, why students work, and what effect working has on student academic success outcomes. Next, the introduction of the co-curricular learning construct will be introduced to consider how campus employment is situated to support student transformative learning, in much the same way as other university-sponsored educationally purposeful activities. Finally, sociocultural theories on workplace learning will be introduced as a perspective from which to explore how student employee learning is supported in the campus employment environment.

\section{Background of the Problem}

As previously mentioned, one way that college students are spending their outside-of-class time in growing and substantial numbers is in various forms of 
employment. The majority of students, nearly $80 \%$ of undergraduates, work in some capacity during college (Perozzi, 2009). Although employment is clearly an important part of today's college student experience, relatively little is understood about the overall effects of working. Campus employment, like any outside-of-class activity facilitated by the university, should be educationally and developmentally purposeful for students, but by and large has not been considered as a learning activity that can be enhanced through effective co-curricular design (Burnside et al., 2019).

\section{Who Works}

Students who work during college come from all types of backgrounds, but there is a marked increase in work intensity, or amount of time spent working, for part-time students, older students, students with lower socioeconomic status, and students from traditionally underrepresented minority groups (King, 2006). Tuttle et al. (2005) demonstrated that African American and Latino students work more hours than their white counterparts. Non-traditional aged students, 25 or over, are also more likely to work than traditional aged college students (Kasworm, 2010). Choi (2018) identified that students who engage in intense work, defined as more than 20 hours per week, were also from the most disadvantaged social backgrounds. Although working in any capacity during college is a near-universal experience, it is clear that some students experience a greater burden of work intensity than others.

\section{Why Students Work}

College students today face a vast array of challenges in working toward degree completion. As college student enrollment has trended upward, the challenges students face have also grown in complexity, including balancing competing priorities, attending 
to financial constraints, navigating a multifaceted educational environment, and many more unique individual circumstances. Currently, there are approximately 17.5 million undergraduates enrolled in higher education institutions, and more students have access to college than ever before in our nation's history (National Center for Education Statistics, 2016). However, higher education has remained a source of social stratification, with students from middle and upper class families experiencing the greatest benefit (Kotzmann, 2018).

Since the 1980s, there has been a growing disinvestment in state funding for higher education, sharply increased by the Great Recession in the late 2000s (Cahalan et al., 2017). Even though the economy at large has bounced back since then, investment in higher education from the states has remained low, leaving institutions with the problem of how to continue to fund the academic opportunities and student support programs they provide. The response, by and large, has been to push the burden of responsibility onto the students themselves through increasing tuition and fees. According to the 2015-16 National Postsecondary Student Aid Study, $72 \%$ of undergraduate students receive some type of financial aid (Radwin et al., 2018). As the costs of attending college continue to rise, the value of federal student grant aid has shrunk from covering $67 \%$ of average college costs in 1975 to only $26 \%$ in 2017 , while those receiving grant aid, in the form of federal Pell Grants, has increased to 33\% from 25\% a decade ago (College Board, 2017). Student loans provide some options for students pursuing a degree, but can be burdensome for many years beyond college. Consequently, students are increasingly reliant on concurrently working while attending college to supplement or cover the cost of their education. 
Although financing college expenses is cited as a primary reason for working during college, students have also expressed other motivations. For instance, Cheng \& Alcántara (2007) found that students in their study initially decided to work to meet financial obligations, but throughout their employment they recognized other compelling reasons to work, including building their network, on the job learning, and greater preparation for entering the workforce upon graduation than their peers who did not work. Other students, primarily from upper-income families, reported their motivations for working included earning additional spending money or gaining work experience (King, 2006). Nunez \& Sansone (2016) examined the meaning of working during college for Latino undergraduate students and found that familial expectations influenced their motivations for working during college.

Whether out of necessity or choice, working while enrolled in college poses a unique set of challenges and opportunities for working undergraduate students. The location and type of employment that students engage in are important mediating factors that will be introduced next.

\section{Where Students Work}

Students work in a variety of positions both on and off campus. According to National Survey of Student Engagement data, $46.5 \%$ of full-time first year students reported working, with $20.7 \%$ working on-campus jobs and $30.9 \%$ working off-campus (McCormick et al., 2010). In the same study, 73.9\% of full-time seniors reported working, with $30.4 \%$ on-campus and 53.7\% off-campus. King (2006) found an even more extreme result, with $91 \%$ of students who work reporting that they work offcampus. Although on-campus student employment is far less common than off-campus 
employment, it is an important environment to study because faculty and staff who supervise student employees have a tremendous opportunity for impact, "given the role that they play in creating employment opportunities, shaping employment conditions, and providing employment supervision and guidance" (McClellan et al., 2018, p. 9).

\section{Effect on Student Success}

One element of student employment that has received substantial attention from researchers is the academic effects of time spent working, otherwise referred to as work intensity. Some studies have found a negative relationship between hours worked and academic outcomes (Callendar, 2008; King, 2006), while others studies have found that moderate amounts of work during college can be academically beneficial (Choi, 2018; Pike et al., 2008). A consistent finding of studies is that working more than 20 hours per week is detrimental for academic achievement (Darolia, 2014; Pike et al., 2008). The effects of hours worked also varies depending on the academic outcome being assessed. For instance, Darolia (2014) found that moderate amounts of work did not have a detrimental effect on grades, but working any amount had a negative relationship with credit hours completed.

Baert et al. (2018) made a distinction between students who have a primary orientation toward school or work to perform a quantitative analysis of the effects of number of hours worked on academic performance. They found that students who were primarily work-oriented worked more hours per week and attained lower grades than their school-oriented counterparts. Conversely, students who had a primary orientation toward school did not experience the same negative relationship between hours worked and academic performance as their counterparts who had a primary orientation toward 
work. This study is an example of the nuance with which student employment research must be treated to fully understand its effect on student success.

A limited number of studies have highlighted the potential benefits and positive outcomes of working during college. McCormick et al. (2010) outlined several benefits that students could receive from engaging in student employment outside of the financial assistance it provides, including applied learning, sense of belonging with peers and faculty or staff members, a deeper engagement with the life of the college, a greater support network, and transferable skill development in several areas, such as time management. In exploring the meaning of work experience for first-generation Latino college students, Nunez (2009) found that students not only appreciated the financial security provided by work, but also found additional value in their employment, including intrinsic satisfaction and exposure to new knowledge and skill development. Similarly, Cheng \& Alcántara (2007) found that students experienced varying degrees of a sense of fulfillment depending on the type of work they were required to do, and that exposure to greater responsibilities in work made the work experience more meaningful. Researchers have also studied the relationship between where students work, onor off-campus, and various aspects of student success. Much of the research that points to the positive aspects of working during college are related to campus employment. Astin (1993), pulling from 30 years of data from the Cooperative Institutional Research Program, concluded that there was a positive effect between campus part-time work and grades. Another study found that working on-campus for 20 hours per week or less had a moderate positive effect on academic achievement and student engagement (Pike et al., 2008). Pascarella and Terenzini (2005) paint a more complicated picture, explaining that 
the relationship between working and student success is not a simple linear relationship. Nevertheless, they also reported that students who worked part-time, on-campus experienced the highest levels of both academic achievement and degree attainment.

Pusser (2010) argues that the continual stream of inconclusive and contradictory research results on the effects of student employment point to a flaw in our attitudes toward student employment. He further goes on to conclude that the value of work for students comes not only from the monetary exchange but also from the personal development that accompanies it. Outside of the financial support that student employment provides, students may also benefit from participating in an applied learning environment, developing a sense of community with peer student staff members and faculty or staff supervisors, a greater connection to the university, a deeper support network, and the development of transferable skills, such as time management (McCormick et al., 2010). As Kinzie \& Hurtado (2017) state "institutional conditions influence student engagement and students' probability of success" (p.36). Administrators and practitioners in higher education have opportunities to influence learning through effectively structuring campus employment experiences, much like any other cocurricular experience, as will be addressed in the next section.

\section{Outside-of-Class Learning}

Increasingly, universities are placing a greater emphasis on developing lifelong competencies, such as leadership skills, in undergraduate students in order to prepare them for civic and societal engagement beyond college (Cress et al., 2001). Keeling (2004) refers to this as transformative learning, in which learning is not limited to the transmission of information from faculty member to student, but rather is "included in a 
much larger context that requires consideration of what students know, who they are, what their values and behavior patterns are, and how they see themselves contributing to and participating in the world in which they live" (p. 9). This type of learning cannot occur without extending intentional learning efforts across campus to support transformative education through "the formal academic curriculum, student life, collaborative co-curricular programming, community-based, and global experiences" ( $\mathrm{p}$. 3). This section will outline the importance of holistically supporting student learning through co-curricular experiences and student engagement before introducing sociocultural theories of workplace learning as a lens for examining the unique contributions of campus employment.

\section{Co-Curricular Experiences}

The expectation for student learning and engagement during college now extends well beyond the classroom and the traditional academic environment. Out-of-class experiences that used to be considered extracurricular are now viewed as making an important co-curricular contribution to student learning, growth, and development when effectively designed (Suskie, 2015). Co-curricular experiences are structured activities that enhance and complement the formal curriculum.

The co-curriculum does what the standard academic curriculum generally does not: It is developmental, transformative, and future-focused. It is also experiential, offering authentic, hands-on opportunities to hone skills, put ideas into practice, and showcase achievements of potential interest to employers. (Rutter \& Mintz, 2016, para. 9)

In other words, effective co-curricular experiences align with and enhance the academic 
curriculum to engage students in educationally purposeful activities and content, contributing to academic achievement and student success.

Student affairs units play a pivotal role in providing effective co-curricular experiences that are developmental for undergraduate students in conjunction with the traditional curriculum. As Keeling (2004) describes, "it is quite realistic to consider the entire campus as a learning community in which student learning experiences can be mapped throughout the environment to deepen the quality of learning" (p. 13). Indeed, examples of extended learning opportunities provided by student affairs abound on campuses, such as living-learning residential centers, integrated service learning programs, internships, health and wellness programs, and many more.

One of the challenges of developing effective co-curricular learning experiences is keeping up with changing student demographics (Suskie, 2015). Today's college students are more diverse and bring a breadth of background characteristics and experiences with them into college. Students do not have the same time or capacity for participation in out-of-class activities as they used to, so it is essential that practitioners develop experiences that meet students' needs; campus employment provides such an opportunity.

\section{Student Engagement}

The student engagement construct has gone through multiple iterations as researchers and practitioners have developed a more thorough understanding of what contributes to student success. Early models of the construct included elements such as time on task, quality of effort, student involvement, social and academic integration, undergraduate education effective practices, and outcomes (Kuh, 2009). Contemporary 
understanding of student engagement "represents both the time and energy students invest in educationally purposeful activities and the effort institutions devote to using effective educational practices" (Kuh et al., 2008, p. 542). Students who are more engaged in college also typically experience greater academic success. Because academic and social engagement are reliable predictors of persistence in college (Engle \& Tinto, 2008), many institutions have successfully developed programs or experiences to enhance these opportunities for students.

Student engagement is best understood as a combination of the effort students put in to their participation in academic and educationally purposeful activities as well as the institutional responsibility for ensuring students have access to these activities. For practitioners, the second component is especially important, because it indicates that the institution holds responsibility for ensuring the conditions for student success are met. Kinzie \& Hurtado (2017) explain that the ways in which institutions prioritize their human and other resources, as well as structuring learning opportunities, is connected to student success. Campus "employment is a longstanding practice that nearly all institutions offer to their students. However, the administration and operations of the practice can vary among campuses" (Burnside et al., 2019, p. 11). Knowing that a large number of students will necessarily participate in campus employment, it is essential to consider the ways in which this experience can most effectively engage students in a manner that supports learning.

\section{High Impact Practices}

Several activities provided on campuses, such as first-year seminars, learning communities, study abroad programs, and capstone projects, have been deemed "high- 
impact" due to their ability to connect academic and co-curricular learning in beneficial ways for students (Kuh, 2008). George Kuh describes high-impact practices as the "institutionally structured student experiences inside or outside the classroom that are associated with elevated performance across multiple engagement activities and desired outcomes, such as deep learning, persistence, and satisfaction with college" (McClellan et al., 2018, p. x). There is much opportunity for on campus student employment to provide similar benefits to students, but the same intentionality must be put into designing the student employment experience as is given to other co-curricular engagement opportunities.

Kuh (2008) outlines the common characteristics that high-impact practices share that makes them so effective. The first is time on task, or the amount of time and effort that students invest in "educationally purposeful activities" (Kuh, 2008, p. 14). Educationally purposeful activities help students gain competence, develop confidence, and become more fully immersed in learning. The second characteristic is deep interaction with faculty and peers in circumstances that allow discussion around substantive content for substantial periods of time. In other research, Kuh (2009) has also stressed the importance of supportive relationships with staff members. The third characteristic is providing opportunities for students to be exposed to diverse people and ways of thinking. Receiving frequent and directed feedback about their performance from authority figures or peers is the fourth characteristic. The fifth characteristic is extending learning beyond the classroom so that students are able to engage in problem solving and teamwork to enact the knowledge gained in the classroom.

\section{Summary}


If learning is truly to be integrated across the entire university, effectively structured campus employment experiences must be part of the discussion. Knowing that academic and social engagement are important predictors of student success, the question remains how to effectively structure campus employment so that students receive the maximum benefit. Sociocultural workplace learning theories provide insight into how onthe-job learning occurs in multiple career fields. Examining campus employment from this perspective may provide additional insights on how learning can best be supported in the campus employment environment.

\section{Workplace Learning}

In the Human Resources Development field, on-the-job learning has caught the interest of researchers for decades. In recent years, the workplace has been dissected to develop a better understanding of the factors that help facilitate learning within that environment, culminating in multiple sociocultural theories of workplace learning. These theories rest on the assumption that learning occurs as a natural part of working, with both formal and informal dimensions supported by organizational, social, and individual factors. Applying sociocultural theories of workplace learning to campus employment provides an additional lens from which to examine how student employees conceptualize learning and to what they attribute learning.

Organizational structure and practices have been found to play an important role in either facilitating or hindering employees' access to learning opportunities (Ashton, 2004; Fuller \& Unwin, 2004). Examples of organizational conditions that influence workplace learning outcomes include environments that support open communication, facilitate opportunities for reflection, provide appropriate challenge and support, and have 
leadership that demonstrate a commitment to fostering a culture of learning (Clarke, 2005; Ellinger, 2005). Social interactions have also been found to contribute to learning at work. Participating in group activities, working alongside others, and receiving guidance from more experienced workers are prime examples of how employees construct meaning through interactions with others (Billett, 2001; Ellinger, 2005). Finally, the influence of individual background, agency and motivation play a role in whether and to what extent employees engage in opportunities for learning in the workplace (Fuller and Unwin, 2004; Billett, 2009).

Although sociocultural theories of workplace learning have explored how organizational, social, and individual factors affect employee learning across multiple types of workplaces, the framework has rarely been applied in the campus employment environment (Carlisle, 2015). However, if the characteristics of high-impact practices (Kuh, 2008) that make them such effective activities for promoting student engagement are closely examined, there is clear alignment with the three central factors in sociocultural workplace learning theories. The amount of time and effort that students extend toward participating in educationally purposeful activities points to the influence of the individual factor. Multiple characteristics align with the social factor, including interactions with peers and faculty members, exposure to diversity of thought, receiving feedback, and engagement in active problem-solving and teamwork. Finally, the organization, in campus employment defined at the departmental level, holds responsibility for intentionally setting up and facilitating the experiences in a way that allows learning to extend beyond the classroom. If the ultimate goal is to effectively deploy campus employment as an opportunity for student learning and development, 
examining it through the conceptual framework provided by workplace learning may provide additional insights.

\section{Purpose of the Study}

The purpose of this study is to explore student employee experiences in campus employment at a large, public Pacific Northwest university to gain a deeper

understanding of the individual, social, and organizational factors that influence learning in the campus employment environment.

"If designed and operationalized effectively, on-campus employment can support students' financial security, while also improving their learning, career-readiness, and persistence outcomes" (Burnside et al., 2019, p. 8). It is time to turn from thinking about the working student experience with a deficit mindset and as a zero-sum equation, where time spent working necessarily cuts into time spent on educationally purposeful activities. Rather, acknowledging the necessity of working during college as a reality of many students, this research will seek to understand the ways in which this aspect of the student experience can also contribute to their learning.

\section{Summary}

In short, with the escalating costs of higher education, student employment is embedded as a mainstay of the American college student experience. Students spend a significant amount of outside-of-class time working, and campus employment has not yet reached its potential in supporting student learning or development. Previous research focusing on the detrimental effects of working during college insufficiently contribute to practitioner understanding of how best to support student learning in the context of campus employment. In a time when students' transformative learning is expected to be 
supported institution-wide, it is prudent to examine the ways in which campus employment can contribute to that goal; sociocultural workplace learning theories provide a unique lens for that exploration.

The remainder of the paper is organized as follows. Chapter 2 provides a literature review, encompassing prior research on student employment, co-curricular learning, student engagment, and sociocultural theories of workplace learning. The study methodology and a brief overview of participants is outlined in Chapter 3. Chapter 4 details the research findings, exploring how personal, interpersonal, and environmental factors interact to facilitate campus employment as a learning experience. Finally, Chapter 5 includes discussion and implications of the research, including introducing the Campus Employment Reciprocal Learning Model. 


\section{CHAPTER 2}

This chapter will build a case that the substantial number of students working their way through college requires a shifting focus in student employment research toward understanding how student employment experiences can be educationally beneficial. The chapter will start with an overview of the student engagement construct, situating student employment alongside other co-curricular activities that contribute to holistic student learning in college. Next, the literature on student employment and its impact on various student outcomes will be discussed. Finally, an overview of sociocultural theories of workplace learning will detail the individual, social, and organizational factors that may contribute to creating a learning environment in campus student employment.

\section{Student Engagement}

The American Association of Colleges and Universities asserts that it is not sufficient for students to emerge from university having only earned a degree; rather, their student experience should provide them opportunities to gain the knowledge, skills, and personal qualities necessary to thrive in a global society (Kuh, 2008). Thus, student learning should be supported by educationally purposeful activities that connect academic learning with out-of-class experiences. Researchers, scholars, and practitioners alike have forwarded student engagement as the leading construct in understanding student success. "Student engagement represents both the time and energy students invest in educationally purposeful activities and the effort institutions devote to using effective educational practices" (Kuh et al., 2008, p. 542).

Student engagement is a multifaceted, complex construct that encompasses the 
intersection of numerous individual and institutional factors. Kahu (2013) refers to student engagement as "an overarching 'meta-construct' that aims to draw together diverse threads of research contributing to explanations of student success" (p. 758). In higher education research, student engagement finds its early roots in Astin's (1993) theory of student involvement, which concluded that the psychological and behavioral time and effort students devote to participation in academic and campus activities is proportionately linked with student development. Over time, the student engagement construct has become more complex as researchers' understanding of which educationally purposeful activities contribute to student success has increased. As the student engagement construct shifted, it included these aspects of time on task, quality of student effort, and student involvement, as well as new considerations of the effects of social and academic integration and effective educational practices on student outcomes (Kuh, 2009).

Currently, student engagement is best understood as a crossroads between the time and effort students dedicate toward participation in educationally purposeful activities as well as the institutional responsibility for ensuring students have access to these activities (Kuh et al., 2008). Thus, both individual and organizational factors are influential in students' engagement in educationally purposeful activities. Student engagement extends beyond the classroom to encompass meaningful outside-of-class experiences, which Kuh (1995) refers to as "the other curriculum" (p. 24). It is the responsibility of institutional agents to ensure that the opportunities provided outside the classroom are accessible to students and structured in ways that effectively evoke opportunities for learning, such as campus employment experiences. 


\section{High-Impact Practices}

A specific subset of engagement activities, referred to as high-impact practices, have been favored by AAC\&U because they "require students to apply and transfer learning across contexts, and to integrate learning from different arenas" (Hansen \& Hoag, 2018, p. 86). High-impact practices share common characteristics, including:

- Allocation of significant time and effort

- Meaningful interactions with faculty and peers

- Exposure to different people and viewpoints

- $\quad$ Frequent, specific feedback

- Opportunities to reflect

- Application of learning to real-world context

- Demonstration of competence

- $\quad$ High expectations (list adapted from Kuh, 2008)

Ten practices have been heavily researched to demonstrate how they effectively promote student success, including common intellectual experiences, learning communities, and service-learning. Two of the high-impact practices have similarities to campus employment and are experiential-based, internships and collaborative projects and research. Currently, campus employment is not considered a high-impact practice, although an effectively structured employment opportunity includes many of the characteristics of high-impact practices (McClellan et al., 2018).

High-impact practices highlight the social and organizational factors that might also be influential in the campus employment environment. For instance, student employees may have the opportunity to receive frequent and specific feedback from their 
supervisors or peers, either through coaching, formal evaluations, or day to day interactions. An organizational example might include requiring that student employees dedicate time for reflection through self-evaluations or providing a safe space to fail.

\section{Educational Outcomes}

Previous research on academic and social student engagement has established its importance as a predictor for numerous student success outcomes (Kuh et al., 2008; Pascarella \& Terenzini, 2005). Kuh (2009) asserts “the greatest impact on learning and personal development during college seems to be a function of institutional policies and practices that induce higher levels of engagement across various kinds of in-class and out-of-class educationally purposeful activities" (p. 688). Implementation of these types of practices can vary considerably, based on how effectively the opportunity is structured.

Kuh et al. (2008) found that engagement in educationally purposeful activities had a statistically significant positive effect on GPA and persistence from first to second year for undergraduate students. Additionally, engagement also had a compensatory effect for students entering college with lower academic achievement (GPA boost), Hispanic students (GPA), and African American students (persistence). Soria (2015) examined the relationship between participation in high-impact practices and academic achievement for working-class college students and found that participation in high-impact practices accounted for a significant amount of variance in the GPA of working class students, though the positive impact varied based on the type of high-impact practice.

Hu \& Kuh (2002) identified characteristics of students likely or unlikely to be engaged in educationally purposeful activities while in college. Characteristics typical of students identified in the engaged category included having high academic preparation 
prior to entering college and parents who had high levels of education. Students classified in the disengaged category were more likely to have parents with lower levels of education and less academic preparation. They also tended to have undeclared majors, be within their first year of college, and perceive that their institution does not value scholarship or intellectual development. In other words, the students who are most likely to benefit from engagement in educationally purposeful activities are the least engaged students.

Museus, Yi, and Saelua (2016) argue for shifting the discourse on student involvement and engagement toward a more culturally conscious direction. They criticize foundational models of student involvement and engagement for being too selfdeterministic and failing to consider the responsibility of the college environment to create conditions in which students from all backgrounds are able to successfully engage. Student employment can provide one such opportunity, providing opportunities for students to engage while also having their financial needs met.

\section{Co-Curricular Learning}

In the seminal student affairs publication Learning Reconsidered, Keeling (2004) advanced the idea that learning in the higher education environment encompasses both cognitive and affective components, defining it as "a complex, holistic, multi-centric activity that occurs throughout and across the college experience" (p. 6). The publication outlined seven essential student learning outcomes and corresponding dimensions: 1) cognitive complexity, 2) knowledge acquisition, integration, and application, 3) humanitarianism, 4) civic engagement, 5) interpersonal and intrapersonal competence, 6) practical competence, and 7) persistence and academic achievement. Learning, through 
this perspective, is not reserved for the classroom but rather permeates throughout the college environment and students' co-curricular experiences.

Co-curricular experiences provide structured activities that are designed to enhance and complement the formal academic curriculum. The organizational structure and practices in the activation of quality co-curricular experiences influences students' opportunities for learning. Suskie (2015) outlined five dimensions of a quality cocurricular experience: focus, relevance, community, evidence, and betterment. Focus involves developing a clear understanding of how to effectively structure the cocurricular experience with intentional learning, growth, or developmental outcomes. It is beginning with the end in mind and having a clear understanding of what students will be able to do or know at the end of the experience as a result of participating. Relevance means that the experience meets students' needs and helps them prepare for whatever is next in their lives. This might include things such as civic engagement or career readiness and requires an understanding of the bigger picture beyond the college experience. Community relates to the in- and out-of-class integration. Learning is not isolated to one experience, so administrators must help students make connections beyond the classroom. Evidence of the effectiveness of co-curricular experiences must be routinely collected and disseminated. Finally, betterment is closing the assessment loop by improving programs using evidence-based decision-making. Effective co-curricular experiences that follow these principles abound on college campuses, but the cocurriculum is rarely found in on-campus student employment, one of the areas in which it could have great impact on a substantial population of students.

In order to understand how campus employment can effectively fulfill its 
potential in engaging students through co-curricular learning, it is imperative to develop a full understanding of exactly how students learn outside the classroom. The next section will outline the theoretical foundations for learning that are relevant to co-curricular experiences, including student employment.

\section{Theoretical Foundations for Learning}

Since the early philosophical musings of Plato, Aristotle, and Confucius, thinkers have been grappling with the question of how people learn (Merriam \& Bierema, 2014). Learning is complex and multifaceted, with both individual and social contributions. As Livingstone (2001) defines it, learning "is the gaining of understanding, knowledge, or skills at any time and anywhere through individual and group processes" (p. 22). The ideas presented below outline the learning theories relevant to learning happening outside the classroom.

\section{Constructivism}

Merriam \& Bierema (2014) define learning as "the construction of meaning from experience," which is the central emphasis of the constructivist conceptual framework (p. 36). The convergence of multiple theories from the works of Piaget, Dewey, and Vygotsky form the foundational roots of constructivism. As opposed to positivists who believe that learning is transferred from experts to novices, constructivists believe that it is only through active learners making sense of their experiences that knowledge is constructed. "Learners, therefore, are not empty vessels waiting to be filled, but rather active organisms in seeking meaning" (Driscoll, 2005, p. 387).

Although constructivism as a whole focuses on this central element of creating meaning through experience, constructivist theorists diverge based on where they fall on 
the continuum of believing that knowledge construction is more of an individual or social process. Driver et al. (1994) provided a helpful distinction between these two viewpoints. Individual knowledge construction is believed to be primarily an internal cognitive activity that is dependent on individuals reconciling previous knowledge with new information gleaned from each progressive physical environmental adaptation. Social constructivism, in contrast, rests on the belief that knowledge and understanding is "constructed when individuals engage socially in talk and activity about shared problems or tasks" (p. 7).

\section{Experiential Learning}

Kolb (1984) identified learning as a central element of being human, calling human beings "the learning species... [whose] survival depends on our ability to adapt not only in the reactive sense of fitting into the physical and social worlds, but in the proactive sense of creating and shaping those worlds" (p. 1). Building off the constructivist tradition, experiential learning occurs through sharing experiences and exchanging dialogue with other people. While the behaviorist theoretical tradition concerns itself with observable behavioral outcomes as the emphasis of learning, experiential learning theory stands in philosophical and epistemological contrast resting on the belief that thoughts are formed and reformed through active participation in experience. Learning is not an outcome, but rather a continual process that allows people to define and modify thoughts based on their experience (Kolb, 1984).

Critics of experiential learning are concerned with the perceived lack of academic rigour and the focus on process rather than content (Kolb, 1984). Yet, experiential learning theory does not negate the contributions of cognitive or behavioral learning 
theories, but rather offers "a holistic integrative perspective on learning that combines experience, perception, cognition, and behavior" (p. 3). Kolb's cycle of experiential learning expressly acknowledges the two domains of perceiving and processing. Information is perceived either through active experimentation, in which the interaction between the individual and the environment produces knowledge, or reflective observation, which involves deep thinking to understand the meaning of situations.

Information is processed either through concrete experience, involving engaging with and acquiring information from others, or abstract conceptualization, which involves thinking and analyzing information.

If one of the goals of higher education is to establish in students a foundation for lifelong learning, that goal can be effectively solidified by participation in experiences that are grounded in experiential learning theory (Kolb, 1984). "The experiential learning model pursues a framework for examining and strengthening the critical linkages among education, work, and personal development" (p. 4). The campus workplace environment itself is a prime environment for experiential learning to enhance and supplement classroom learning and foster individual development.

\section{Informal Learning}

Typically when learning is discussed in American culture, the first thing that comes to mind is formal learning that is structured and takes place within the confines of educational institutions. "Indeed, we have been so conditioned to thinking of learning as something that takes place in an educational institution that our learning at work or in our everyday life does not seem to count as part of our learning" (Merriam \& Bierema, 2014, p. 16). However, learning is not a process reserved exclusively for the classroom. 
Informal learning encompasses a much broader and more prevalent type of learning that is embedded in everyday contexts. The nature of informal learning is both spontaneous and unstructured, and as such, it can occur anytime and anywhere (Merriam \& Bierema, 2014).

The workplace provides a prime setting for informal learning, and Kim et al. (2004) estimated that approximately $70 \%$ of the learning that takes place in that environment is informal. However, studying informal learning poses a challenge for researchers due to its tacit nature (Eraut, 2007). Thus, it is important to qualitatively explore the ways in which students experience informal learning within the campus employment environment to enhance understanding.

\section{Triadic Reciprocal Determinism}

Bandura (1986) introduced a model of reciprocal determinism within social cognitive learning theory, which proposed that learning takes place in a social environment as a result of bilateral relationships between an individual's cognition and other personal factors, the environment, and behavior. The interactional links between personal, environmental, and behavioral factors influence how learners make meaning in a social environment. The link between personal and behavioral reflects the interaction between what individuals think, believe, or feel and how they behave. The link between personal and environmental reflects the interaction between personal characteristics and environmental influences, in which learning occurs through social interaction influencing thoughts and vice versa. The link between environmental and behavioral reflects the influence of environmental conditions on an individual's behavior and the reverse. Although each of the three factors that are part of the triad influence outcomes, the 
strength and effects of the influence differ depending on the situation (Bandura, 1986).

\section{Summary}

Multiple aspects of the student experience, both within and outside the classroom provide opportunities for meaningful contribution to student success in college.

Constructivism, experiential learning, and informal learning theories help contextualize the role of co-curricular learning experiences, emphasizing that learning can happen in any number of circumstances through practice, reflection, and the co-construction of meaning. As Fried (2017) describes it, learning "is a process that occurs when people have new experiences, reflect on the experiences, and derive some verbal description of the meaning, value, and possibly skills associated with those experiences” (p. xv).

Students' academic and social engagement in educationally purposeful activities is a reliable predictor of student success, yet the students who stand to benefit the most from participation in educationally purposeful activities participate the least $(\mathrm{Hu} \& \mathrm{Kuh}$, 2002). As the next section will outline, one reason for this is that current college students are working with greater frequency and intensity. Student employment has the potential to provide an educationally purposeful experience for students who spend significant time engaged in work. In order to understand how campus employment can effectively fulfill its potential in engaging students through co-curricular learning, it is imperative to develop a full understanding of exactly how learning occurs in the campus workplace.

\section{Student Employment}

The purpose of this study is to explore the ways in which students experience learning within the campus employment environment. The large body of literature on student employment is vast and varied, with researchers considering such things as work 
intensity, location, need, and relevance to understand the overall effect of working on students' college experience. Yet, previous research on the topic has neither found simple nor consistent explanations to describe the relationship between student employment and educational outcomes (Pike et al., 2008; Pusser, 2010). This section will provide an overview of student employment research, including which students work and why, common conceptions of student employment, and current gaps in the literature.

\section{Who Works}

Since the inception of American higher education, students have simultaneously worked while attending college; however today's college students are working with greater frequency and at greater intensity than at any other point in history (McCormick et al., 2010; Tuttle et al., 2005). The working student population grew from $40 \%$ to $80 \%$ between 1961 and 2000 (Tuttle et al., 2005). Data from the National Center for Education Statistics in 2009 showed that $79 \%$ of undergraduate students worked at least one hour per week (Perozzi, 2009). At four-year undergraduate institutions, approximately twothirds of students were employed, with a quarter working 35 hours per week or more (McCormick et al., 2010). McCormick et al. (2010) also found that the total percentage of the student body working increased from their first-year to their senior year, from $46 \%$ to $75 \%$. As King (2006) concluded, "working while enrolled is perhaps the single most common major activity among America's diverse undergraduate population" (p.2). Clearly, employment during college is a near ubiquitous student experience, and all the evidence suggests that this is not a passing trend.

Although a broad swath of the undergraduate population engages in work, previous studies have found that the influence of work differs depending on student 
demographics. As Perna (2003) explains, students who work come from various backgrounds, have different characteristics, and experience unique challenges, so they cannot be treated as a homogenous group. Students from backgrounds that have been traditionally underserved by higher education institutions are more likely to be immersed in work during college. Tuttle, et al. (2005) highlighted that African American and Latino students frequently work with greater intensity than their white counterparts. Similarly, first-generation students are more likely to work during college and tend to work with greater intensity than their peers (McCormick et al., 2010). Students over age 25 bring a multitude of additional experiences and responsibilities with them into college, and as Kasworm (2010) pointed out, are more likely to engage in employment and work full time than their younger counterparts.

\section{Why Students Work}

In a review of the National Postsecondary Student Aid Study, King (2006) found that the primary reason students cited for working during college was to cover the cost of tuition and fees. Within the 10 year period from 1998 - 2008, the average cost of tuition and fees at public four year institutions increased by 50\% in constant dollars; however, within that same period, average family income increased by only $3 \%-5 \%$ for families in the lowest socioeconomic quartiles (Perna, 2003). Even when they received substantial financial aid grants and loans, many students were still left with a significant funding gap to cover the cost of tuition and fees (Baum, 2003). As Baum (2003) concluded, with the price of college continually increasing more rapidly than their income, "more students are certain to find work a necessity rather than a choice" (p. 3).

While paying for educational costs was the most commonly cited reason for 
working during college, it was not the only reason students reported for engaging in work. Students primarily from upper-income families described their primary reasons for working included earning additional spending money and gaining work experience (King, 2006). Older students typically carried greater responsibilities and family obligations than younger students, which added an additional layer of complexity to their reasons for working while concurrently attending classes, and in some instances, work was also tied to their personal identity (Kasworm, 2010). Perna (2003) also concluded that undergraduate students may use work experiences during college to explore career options.

In one of the few qualitative studies that examined student employment beyond academic outcome variables, Cheng \& Alcántara (2007) explored student perceptions of working in the context of their college experience. Students expressed a dichotomy between juggling priorities related to work and study, but also finding meaning in their work. The further engaged students were in their work, the more meaningful they found their work experience. Although many students in this study were initially motivated to work to meet financial obligations, their thinking expanded to recognize other benefits, such as on-the-job learning, interactions with others, networking, and a feeling of being ahead of the game compared to their peers in workplace preparedness. Rather than impacting their academics, as some studies have shown before, students in this study expressed that their social lives were most impacted. When reframed, students also conceded that working enhanced their social skills and competencies in a way that regular socializing did not. Overall, the study found when students found meaning or value in their employment, they considered it an integral part of their college experience. 


\section{Impact of Work}

The amount of time students engage in work and the related effects on engagement and academic outcomes is another part of student employment research that does not show any simple relationships nor consistent outcomes. This section will explore the research related to the effects of work on academic and cognitive and developmental outcomes before turning to research that examines a more nuanced understanding of those outcomes dependent on type of employment.

\section{Academic Outcomes}

The increase in the percentage of the student population working during college directed much of the research efforts to focus on understanding the effects of work intensity, or the amount of time students spend engaged in work per week, on academic outcomes. However, as McCormick et al. (2010) pointed out, studies on work intensity are so varied in their methodology, variables, and populations studied that it is challenging to draw generalizable conclusions.

Kulm \& Cramer (2006) found a curvilinear relationship between hours worked and academic outcomes, with students who worked less than 15 hours per week experiencing a boost in grade point average compared to their peers who worked more than 15 hours per week or did not work at all. King (2006) similarly found a positive association between work and GPA for students working less than 20 hours per week and a negative association for students working more than 20 hours per week. Pascarella \& Terenzini (2005) also reported a non-linear relationship between engaging in work and academic outcomes. Hovdhaugen (2015), exploring the experiences of Norwegian students, similarly discovered that students who worked less than 20 hours per week were 
not at any greater risk of dropout than their peers who did not work at all, but students working over 20 hours per week were at increased risk of dropout. Logan et al. (2016) also found that working over 20 hours per week was negatively associated with grade point average, but only for students working off-campus jobs.

In contrast, Baert et al. (2018) hypothesized that whether students were primarily oriented toward school or toward work might be a factor that affected the relationship between hours worked and academic performance. They found an overall negative relationship between hours worked and academic performance, as defined by credit hours completed; however, the effect was lessened for students who had a primary orientation toward school rather than work. Similarly, Callendar (2008) pulled a random sample of final-year students in the United Kingdom and conducted a multiple regression study to understand the effects of term-time employment on academic grades. The researcher found a negative relationship between the number of term-time hours worked and academic achievement.

In a unique study, Choi (2018) considered background characteristics, including socioeconomic status and pre-college academic achievement, to define students' propensity to work during college and explore the effects on college dropout. Students with a high propensity to work were those who were likely to engage in employment during college in order to pay for tuition and fees, rather than simply to gain work experience. The overarching trend identified in the study is that students who engaged in intense work, over 20 hours per week, within their first year were also from the most disadvantaged social backgrounds. As students' propensity to work increased, so did the risk of dropout. However, the effects of intense work were most deleterious for students 
from the most advantaged social backgrounds and least detrimental for students from the most disadvantaged social backgrounds. As such, the students who exhibited a need to work in college did not experience as much risk for drop out as other working students. This is an important finding because it exemplifies the nuance with which student employment must be explored in order to fully understand the effect of working during college on specific student populations.

\section{Cognitive and Developmental Outcomes}

Although student employment researchers have placed far more emphasis on the effects of student employment on academic outcomes, a limited number of studies addressed the effects of working during college on students' cognitive or developmental learning. Strauss and Terenzini (2007) evaluated the influence of both in- and out-ofclass experiences on engineering student gains in analytic and group work skills. They discovered that working during college positively influenced gains in both analytic and group work skills at the end of the program. Lundberg (2004) also examined self-reported gains in broad measures of learning, comparing students who worked off campus and students who did not work, and found no difference between the populations.

In seeking to understand the effect of an array of student involvement activities, including student employment, on cognitive development, Gellin (2003) conducted a meta-analysis of related literature. The study concluded that students who worked during college experienced a moderate gain in critical thinking compared to their peers who did not work. However, this study did not distinguish between the type of employment students held nor was it able to explain discrepancies in cognitive development according to numbers of hours worked, limiting the conclusions that can be drawn. 


\section{Type of Work}

Students work in a wide range of positions during college, yet previous research on student employment has only just begun to account for the intricacies of various types of employment. One of the broad distinctions that has been made in student employment research is in considering the effects of working on-campus versus off-campus positions. According to King (2006), less than 10 percent of students work in on-campus positions. McCormick et al. (2010) found slightly higher numbers, with one in five first-year and one in three senior-year students reporting they worked on campus. Although students are more commonly employed in off-campus jobs than on-campus, there is greater opportunity for higher education practitioners to influence the on-campus student employment environment and shape an educationally purposeful experience for student employees.

Previous campus employment research has shown that working on campus positively contributes to the student experience. Furr \& Elling (2000) found that students employed on campus were more engaged in their campus community, were more likely to join a club or organization, and experienced more frequent interaction with faculty members than their peers who worked off campus. In studying leadership development, Salisbury, Pascarella, Padgett, and Blaich (2012) found that students working off campus experienced higher gains in leadership capacity than campus student employees.

The research on campus employment has also demonstrated that students working on campus persist at higher rates than their peers. In his review of the National Postsecondary Student Aid Study, King (2006) discovered increased levels of persistence and degree completion in students who worked 15 hours or less in campus jobs, but noted 
that few working students are employed in these positions. Beeson \& Wessel (2002) compared persistence and graduation rates for students from a single institution who worked on campus their freshmen year compared to students who did not work on campus and similarly found that on-campus student employees persisted to graduation at higher rates.

The effects of campus employment on a number of student engagement factors has also been explored in the literature, most commonly through analyzing data from the National Survey for Student Engagement (NSSE). Pike et al. (2008) used this data set to better understand how the relationship between student employment and engagement in educationally purposeful activities for first-year undergraduate students affected academic achievement, as defined by grade point average. The additional factors of hours spent working and type of employment were also considered. They found a direct and negative relationship between working 20 hours or more per week and academic achievement, regardless of type of employment. However, students working 20 hours or less had similar academic achievement to students who did not work at all. Students working on campus for 20 hours per week or less also experienced a moderate positive effect on academic achievement, but the effect was diminished when student engagement factors were taken into account.

In their analysis of NSSE data, Pike et al. (2008) also discussed the relationship between engagement in educationally purposeful activities in college and academic outcomes as a mediating factor in understanding the relationship between working during college and academic outcomes. They discovered that working on campus for 20 hours per week or less was positively related to engagement measures, with particularly strong 
relationships in the Student-Faculty Interaction and Active and Collaborative Learning measures. While working 20 or fewer hours off campus was also positively related to those two measures, it was negatively related to the Supportive Campus Environment measure. Taken together, they found a statistically significant positive relationship between working 20 or less hours on campus and academic achievement.

NSSE data was also used by McCormick et al. (2010) to analyze the relationship between student employment, engagement factors, and academic outcomes. NSSE has five benchmarks of effective educational practices: academic challenge, active and collaborative learning, student-faculty interaction, enriching educational experiences, and supportive campus environment. The researchers found a positive relationship between each of the five benchmarks and working an on-campus job, compared to students who did not work, with especially high correlation in the benchmarks of student-faculty interaction, active and collaborative learning, and enriching educational experiences. Surprisingly, and contrary to other research, the effect was magnified for students who worked more than 20 hours per week on campus. Students who worked in off campus positions experienced smaller, but still positive, gains in the benchmarks, with the exception of supportive campus environment for those students who worked more than 10 hours per week. The findings related to hours worked and self-reported GPA were consistent with other research. Students who worked 10 hours or less on-campus reported slightly higher GPAs, while those who worked on-campus for 20 hours or more or offcampus for 10 hours or more reported lower GPAs.

Not all campus employment experiences are the same, and a limited number of researchers have delved into the intricacies of specific employment experiences. Fede et 
al. (2018) focused their research on a unique on-campus employment experience that had significant off-campus work to examine the transferable skill development and academic success of student employees. Previous student employees were purposefully sampled to participate in a survey measuring traditional markers of student success, such as selfreported GPA and graduation date and transferable skill development. On the traditional success indicators, former student employees reported higher graduation rates than their peers at the institution and the national average. Former student employees also selfreported development in many transferable skills during their employment, including empathy, communication, and problem-solving. As Fede et al. (2018) concluded, engagement in this type of experiential education complements the academic experience through transferable skill development, although this study did not identify the factors of the employment experience that contributed to the self-reported growth.

Kuh (2009) has alluded to the potential of campus employment experiences to meaningfully enrich student employees' learning with proper structure and reflective opportunities. Previous research has demonstrated that through campus employment, student employees receive a number of beneficial outcomes. However, few studies have specifically considered the learning potential of campus employment experiences. It is clear that campus employment poses some benefits for student employees, yet much more could be done to ensure the experience provides opportunities for learning.

\section{Assumptions}

One of the major guiding assumptions in much of the student employment literature is of zero-sum thinking, where time spent working necessarily takes away from time spent on academic pursuits (Baert et al., 2018). This perspective is so pervasive 
throughout student employment research, that it has left little room for consideration that student employment might positively contribute to a student's college experience or success thereafter. That type of research can certainly inform how student employment is approached on a state or institutional policy level, yet does little to direct student employment practices in the workplaces where students are actually working their way through college.

\section{Learning in Student Employment}

Carlisle (2015) identified "seven salient concepts of learning" from the student employment research that point to its potential to contribute to student learning and development (p.49). First, student employees engage in learning through carrying out routine and non-routine job responsibilities and tasks (Carr, 2005). Second, knowledge is socially constructed through relationship-building and engagement in dialogue (Carr, 2005; Lundberg, 2004). Third, opportunities for reflection and continual self-assessment contribute to the learning process (Carr, 2005). Fourth, although learning is often considered in terms of outcomes, it is important to consider the learning process itself and its developmental aspects (Carr, 2005). Fifth, student employee perceptions of their student employment experience are influenced by the student themself, their parents, and their employer (King, 2006; Nunez \& Sansone, 2016). Sixth, student employees who understand the value and find meaning from their employment experience are more motivated to learn from the experience (Cheng \& Alcántara, 2007). Finally, the selfconfidence that student employees possess is related to their learning and development (Carr, 2005).

Winkler (2009) stressed the need for further research aimed at understanding the 
ways in which student learning is promoted or hindered through organizational structures. A thorough exploration of campus employment is needed to understand the ways in which the employment experience can be effectively structured to deepen learning. Further, Pomerantz (2006) implored student affairs units to adopt the student engagement paradigm to effectively optimize opportunities for students to participate in structured, intentional learning environments build out of student learning outcomes.

\section{Campus Employment Programs}

Campus employment has largely been considered for its exchange value, rather than its learning potential, but some universities have started implementing programs to address this gap. "Campus employment is rarely structured to focus on learning and, like a number of college experiences, may lack integrating structures that facilitate student reflection and transfer of learning" (Hansen \& Hoag, 2018, p. 87). As the expectation for engagement opportunities across the spectrum of college activities has grown, campus employment as a learning and developmental experience has slowly started to become a point of focus on a limited number of college campuses which have worked toward formalizing the learning experience in campus employment at the departmental, divisional, or institutional level.

Broughton \& Otto (1999) assessed the effectiveness of the Leap to Excellence Acceleration Program (LEAP), a student employee training program designed to formally develop student employees according to an established set of intentional learning outcomes, including leadership, creativity, customer service, problem solving, multicultural recognition and appreciation, and communication skills. The implementation of LEAP involved a collaborative goal setting session between student 
employees and their supervisors, followed by ongoing mentorship, feedback, and evaluation. Using a programmatic questionnaire, supervisors provided their perceptions of student development within the six learning outcomes and indicated growth in student employees in the domains of customer service, leadership, and problem-solving skills. However, when feedback from student employees was collected, they only identified growth in the leadership domain. Broughton \& Otto (1999) laid a foundation for the importance of developing learning outcomes and intentionally infusing learning throughout the employment experience, highlighting that time and effort is needed from both supervisor and student employee to facilitate learning experiences.

IOWA GROW $^{\circledR}$ (Guided Reflection on Work) out of the University of Iowa pulls in components of integrated learning, reflective learning, and transfer of knowledge in a unifying framework. “The IOWA GROW ${ }^{\circledR}$ intervention consists of brief, structured learning-centered conversations between student employees and their supervisors" (Hansen \& Hoag, 2018, p. 88). The twice per term conversations between supervisors and student employees center on four core questions:

1. How is this job fitting in with your academics?

2. What are you learning here that is helping you in school?

3. What are you learning in class that you can apply here at work?

4. Can you give me a couple of examples of things you've learned here that you think you'll use in your chosen profession?

The intent of the IOWA GROW ${ }^{\circledR}$ framework is to guide student employees to reflect on the intersection between academic and workplace learning and consider applicable skills that can be carried into future employment. Students who participated in 
IOWA GROW ${ }^{\circledR}$ were better able to articulate skills gained from participation in student employment than other student employees who did not participate in the intervention (Hansen \& Hoag, 2018).

"The degree to which a particular on-campus employment opportunity serves as a high-quality, developmental experience can depend on the various work conditions, processes, and policies an institution has in place" (Burnside et al., 2019, p. 1). Programs such as LEAP and IOWA GROW ${ }^{\circledR}$ demonstrate the shifting perspective on campus employment from transactional relationship to growth opportunity. Through the development of a deeper understanding of precisely how learning is transmitted within campus employment, the effectiveness of facilitating student employment as a learning opportunity will continue to be improved.

\section{Summary}

Working during college has become a big part of the American undergraduate college experience. With so many students working while attending college, it is important to understand how the work experience affects college student learning, development, and growth; yet research on the topic has been inconclusive at best, and at worst, has perpetuated a deficit mindset toward student employment, assuming that student employment and academic learning are necessarily in competition (Pascarella \& Terenzini, 2005; Pike et al., 2008).

Although the opportunity to influence off-campus work conditions is limited, higher education administrators have the opportunity to influence the on-campus work experience through the administration and oversight of student employment programs. On-campus student employment reaches a broader portion of the student body than most 
other university-sponsored activities, and it has the potential to be a primary engagement opportunity for student employees. Continued research is needed to understand how the campus workplace environment, including culture, organizational and social structures, and individual motivational factors contribute to or hinder student employee learning. The next section will explain how looking at student employment through the lens of workplace learning theories can contribute to a deepening understanding of how learning occurs in the student employment environment.

\section{Workplace Learning}

As Cullen et al. (2002) contend, "looking at work from the perspective of its learning potential is fundamentally different to looking at it simply in terms of competencies needed in order to perform the job well”' (p. 2). An increasingly complex understanding of the emerging workplace learning construct has developed over the past two decades within the Human Resource Development field of study. Analyzing learning through this construct "offers new insights into the multi-dimensional nature of learning through participation in employment activities" (Carlisle, 2015, p. 52). This section will provide an historical overview of the evolution of workplace learning theories, from its primarily formal, individualistic origins to the multifaceted construct that is understood today. Workplace learning research provides an additional theoretical lens from which to explore learning in the campus employment environment.

\section{Theoretical Origins}

Early vocational education was influenced primarily by behaviorism, resting on the "flawed assumption [that] learning is to be thought of solely in terms of observable behaviors, since other terms commonly associated with learning, such as thinking, 
knowing, and understanding, refer to activities that are inaccessible to direct observation" (Hager, 2011, p. 18). The rationale behind this required breaking down the work into codifiable behaviors and providing formal training for employees to perform these behaviors with appropriate stimuli and reinforcement (Hager, 2011). In a period of increasingly complex and unpredictable work, it is apparent that this simplified way of conceiving vocational learning does not hold up.

Workplace learning theory saw its first major surge in the 1970s through the 1990s, heavily influenced by psychological and cognitive theories of learning. Major themes that typified this period of workplace learning research included a focus on individual learners, attention to the rational, cognitive aspects of the workplace, reflective thinking influencing future action, and the treatment of learning as a product rather than a process (Hager, 2011). Importantly, and in contrast to more recent research in the field, the learning product was considered independent of the context in which it was learned in many early theories (Hager, 2011). For instance, Argyris \& Schon (1978) introduced the concept of reflective learning into the workplace environment, culminating in the enduring image of the reflective practitioner who is able to nimbly learn through reflective practice. Watkins and Marsick (1992) expanded the reflective learning concept to also include informal or incidental learning that occurs when workers engage in nonroutine experiences.

\section{Sociocultural Theories of Workplace Learning}

In the 1990s, workplace learning research started being influenced by sociocultural theories based in sociology and social anthropology. Stasz (2001) contrasted the traditional economic perspective of learning with the sociocultural perspective. In the 
economic perspective, skill is viewed "as an attribute that is amenable to quantitative measurement and has objective character independent of the observer" (p. 387). This viewpoint is readily apparent in schools, where testing is used to determine mastery of subject matter, and in workplaces, where individuals are deemed qualified for jobs based on meeting skill requirements. In contrast, the "sociocultural perspective shifts the focus of inquiry from individuals to interactive systems or social settings that are larger than the behavior and cognitive processes of a single person" (p. 388). The emphasis of inquiry moves from what skills are gained to how learning and shared meaning-making occurs within the workplace environment. Fuller and Unwin (2011) reaffirm this approach, saying it "provides a framework for capturing the complex pattern of social relations and organizational factors which influence how people learn at work and how this learning is valued, fostered or limited" (p. 50).

Sociocultural theories of workplace learning differ from their predecessors influenced by psychological and cognitive theories in three major ways (Hager, 2011). First, sociocultural theories of workplace learning emphasize the social nature of learning, rather than the individual. Second, the ongoing process of "learning as participation" is of greater importance than learning as a product (Fuller \& Unwin, 2011, p. 50). Third, sociocultural theories heavily consider the contextual influence of specific workplaces on learning. Each of these distinctions will be further discussed in the following sections.

\section{Social Nature of Learning}

Rather than focusing solely on the individual learner, the social aspects of learning are given greater prominence in sociocultural workplace learning theories. Lave 
and Wenger (1991) best described learning as "an integral and inseparable aspect of social practice" (p. 31). They introduced the concept of workplaces as communities of practice, whereby situated learning occurs through relational participation, rather than within an individual's own head:

Learning viewed as situated activity has as its central defining characteristic a process that we call legitimate peripheral participation. By this we mean to draw attention to the point that learners inevitably participate in communities of practitioners and that mastery of knowledge and skill requires newcomers to move toward full participation in the sociocultural practices of a community. (p.

One of the main components of their theory centers on the shift from the individual learner to learning as a social process involving groups of people engaging in thinking and meaning-making while together engaged in activity. Individuals learn as they move from being outsiders into full participants.

Although some sociocultural workplace learning theories completely discount the individual learning experience, as Lave and Wenger do, many others consider more holistically both the individual and social experiences of learning (Billett, 2002; Eraut, 2000; Fuller \& Unwin, 2003). Billett (2001) insists that there is "an interdependence between individuals and social practices" (p. 68). Under this assumption, how and to what extent individuals participate in opportunities to learn provided by the workplace is influenced by their individual agency. Some of the individual factors at play include personal histories, alignment between individual interest and perceived value of the learning opportunity, and individual perceptions of the underlying intent behind learning 
opportunities provided by the workplace.

\section{Learning as a Process}

The second major divergence in sociocultural workplace learning theories is the consideration of learning as an ongoing process rather than a product. Wenger (2000) defines learning as "an interplay between social competence and personal experience. It is a dynamic, two-way relationship between people and the social learning systems in which they participate. It combines personal transformation with the evolution of social structures" (p. 227). As this description makes clear, Wenger is not necessarily concerned with what, exactly, is learned but rather with the process that induces learning.

Engeström (2001) considered the workplace as an activity system, encompassing cultural norms and social rules, in which learning occurs primarily through the resolution of tensions and contradictions throughout the system. He developed a theory on expansive learning that is firmly rooted in process. He suggests that much of what needs to be known in the workplace cannot be identified ahead of time, but rather emerges in time through normal work engagement. Workplace learners "construct a new object and concept for their collective activity, and implement this new object and concept in practice" (Engeström, 2011, p. 87). This process merges knowledge and skill acquisition with new activity design to meet the demands of a complex workplace.

Routine work activities have also been found to contribute to the learning process within workplaces. Eraut (2004) considered how workers were learning knowledge and skills in the workplace and found that there were four primary work activities that routinely contributed to learning: participation in group activities, working alongside others, tackling challenging tasks, and working with clients. The success of these efforts 
was mediated by the quality of relationships and context within the workplace.

Additionally, specific learning activities were found within the primary work learning processes, including "formal study, listening, observing, reflecting, practicing and refining skills, trial and error, supervision or coaching, and mentoring” (p. 267). Finally, Eraut identified factors that affect learning in the workplace, recognizing that context significantly influences the relative importance of each factor. The interrelationship between challenge, support, and confidence speaks to both environmental and personal aspects of learning. The broader contextual factors that impact learning at work included how work was allocated and structured, performance expectations, and relationships.

\section{Learning in Context}

Finally, sociocultural-based theories rest on the belief that workplace learning is "significantly shaped by social, organizational, cultural and other contextual factors" (Hager, 2011, p. 23). Billett (2001) explained that the workplace learning environment consists of both organization elements, such as culture, management, policy, and procedures, as well as individual motivations to learn. As such, employee learning occurs as a result of the interdependence between what the workplace provides in creating conditions for learning and how the individual engages in these environments. He uses the concept of affordances to describe the ways in which the workplace supports learning opportunities through providing experiences, "access to activities, and guidance from more experienced and expert practitioners" (Billett, 2009, p. 835).

Fuller and Unwin (2004) characterized workplace learning environments based on their placement on an expansive restrictive continuum. The continuum is based off of a set of workplace features that either facilitate or hinder employee learning. Fuller and 
Unwin considered both organizational and pedagogical features of the workplace environment in developing the continuum in order to capture "organizational context and culture" as well as "understandings of how employees learn through different forms of participation" (p. 51). Workplaces on the expansive side of the continuum facilitate learning through providing opportunities for employees to engage in dialogue and problem solving horizontally across the breadth of the workplace. Professional development is seen as benefiting both the organization and the individual and expertise is not limited to hierarchical positional leadership, but rather spread throughout the organization. Substantial time is set aside for reflection and further supported growth through mentoring and coaching. In contrast, the restrictive work environments provide little opportunity for employees to work outside their portfolio. Skill development is specified to the required task at hand and is directed top-down by designated experts. Fuller and Unwin's research points to the importance of the work environment in facilitating access to learning opportunities, which come through social interactions and engagement in the work itself.

Billett (2001), drawing on a series of previous studies outlined a framework for effectively constructed workplace learning environments. Elements of the workplace that contribute to learning included opportunities for workers to engage in routine and nonroutine work tasks, access to guidance provided by more expert workers, and indirect support through observations of other workers and the work environment itself. Although learning within the workplace environment to some extent is inevitable, an effectively structured workplace curriculum can help maximize opportunities. Billett (2001) proposed that an effective workplace curriculum contains four elements: facilitated 
movement from low-to high accountability work activities, access to knowledge that could not be discovered without facilitation, direct guidance from individuals with more expertise, and indirect guidance from the social and physical environment.

\section{Summary}

Sociocultural theories of workplace learning help bring some definition to the complexity of factors that influence learning within the workplace environment. Applying these theoretical perspectives to the campus employment environment would undoubtedly expose an equally complex system. The next section will conceptualize workplace learning in order to form the basis from which to explore how learning is supported or hindered in the campus employment environment.

\section{Conceptualizing Workplace Learning}

When considering the ways in which workplace learning occurs through sociocultural theories of workplace learning, the influence of individual, social, and organizational factors are inextricably linked. Careful consideration of each aspect contributes to the overall picture of workplace learning. As Fuller \& Unwin (2004) stated, "an over-emphasis on the structural character and environmental features of organisational context can underplay the role of individuals' backgrounds, prior attainments, attitudes, wider experiences and agency, whereas an over-emphasis on the individual can divert attention from the influence of the organisational and wider institutional context in which learning at work occurs.” (p. 12). This section will explore the literature related to the individual, social, and organizational factors that influence workplace learning.

\section{Individual}


The effectiveness of workplace learning is partially dependent on how and to what extent individuals engage in the learning process. Billett (2009) refers to the epistemologies of learners, referring to their individual histories, knowledge, preferences, and tendencies that they bring with them into the workplace. "Different learners perceive the same opportunities differently and react towards them differently, because of their differing dispositions. Indeed, the very nature of any opportunities the workplace may offer for learning depends partly upon those dispositions." (Hodkinson \& Hodkinson, 2004, p. 176). Without a willingness to engage as agentic learners, even the most wellstructured workplace learning opportunities will leave learning unrealized for some employees. On the other hand, employees who are highly agentic learners will find opportunities for learning in even the most unstructured workplace experiences.

Further emphasizing the influence of the individual's willingness to engage in workplace learning, Billett (2001) claimed "there is an independence between individuals and social practices" (p. 68). How and to what extent individuals participate in workplace learning activities is influenced by their individual agency. Factors influencing an individual's willingness to engage in learning at work include personal histories, alignment between their individual interest and perceived value of the learning opportunity, and individual perceptions of opportunities afforded by the workplace to contribute to their learning. In another study, Billett (2009) used the concept of engagements to describe the importance of individual motivation in workplace learning. "Engagement refers to how students engage with and learn through what they are afforded" by the workplace (Billett, 2009, p. 835). In other words, to what extent do employees take advantage of the learning opportunities provided by the workplace. 
In studying how employees conceptualize learning at work, Collin (2002) identified individual attitudes and behaviors that relate to how employees learn. Learning through doing the work itself is an important aspect of workplace learning. When employees engage in problem-solving or recognize competencies and knowledge needed to do the job, learning is induced. Additionally, drawing on previous experience or learning from past mistakes and connecting it to the task at hand is often a subconscious process that contributes to learning. The acquiring of new information, experimentation, and creative thinking that go into taking on a new work task or project facilitate learning. Finally, employees also contribute to workplace learning when they engage in foundational formal education or bring in and synthesize knowledge gained outside the work context.

The concept of an individual's "learning territory" was introduced by Fuller and Unwin (2004). It refers to the ways in which personal backgrounds, educational experiences and aspirations shape the extent to which individuals respond eo learning opportunities at work. They demonstrated that even when the workplace environment provides structured and sufficient opportunities for learning, the employee must be an active participant in developing their own capacities for learning to occur.

Even in describing individual factors, it is nearly impossible to separate out the influence of the workplace and the people in it. For instance, Clarke (2005) explored the workplace conditions that influence learning in healthcare organizations and outlined a number of individual opportunities for on-the-job learning based on his findings, including learning through trial and error, observation of others, reading or self-study, taking on challenging projects, and job rotation. Although these activities seem to be self- 
directed, as further pointed out in the next section, the organization itself plays a role in ensuring that opportunities for employee engagement in these activities are available.

Eraut (2007) considered both sociocultural and individual theories in the development of a typology of early career learning. The individual perspective encompasses "what individual persons bring to situations that enable them to think, interact, and perform" (p. 406), including their capabilities and understandings. Eraut's typology separated out primarily work processes, primarily learning processes, and learning activities that could happen at any time. He found a number of employee behaviors that support individual learning in the workplace, including proactively seeking information, locating resource people, asking questions, accessing prior knowledge, and demonstrating social savvy. Additionally, Eraut called out an employee's confidence in his or her ability to complete a specific task or hold a role and their personal agency as factors that influence workplace learning.

Hackman and Oldham (1976) also stressed the important contribution made by both organizational and individual factors, which lead to beneficial outcomes, such as increased employee motivation, performance, and satisfaction. Through their Job Characteristics Model, they proposed three psychological states which cause individuals to experience internal motivation in their work: (1) The worker must find the work personally meaningful within their internal values system. (2) The worker must feel a sense of personal responsibility for the work output that is based on their own efforts, rather than that of other workplace factors. (3) The worker must effectively see the results of his or her efforts. Five job characteristics cultivate the emergence of the psychological states. Meaningfulness is facilitated through skill variety, task identity, and task 
significance; responsibility is prompted through autonomy; and results are facilitated through feedback. Collectively, the interaction of these three elements constitutes the overall motivating potential of a job. However, the Job Characteristics model adds one additional element of individual growth needs, which represents how compelled an individual is to engage in growth and development or achieve self-esteem and selfactualization through work. The higher an individual falls upon the growth needs continuum, the more likely they are to experience the three psychological states when situated in an enriched workplace environmnet.

\section{Organizational}

The second element that helps contextualize learning through work is organizational factors, including the ways in which the work is stuructured, opportunities within, and the overall culture. Organizational structure and practices may also impact individuals' motivations to learn in the workplace. Ashton (2004) sought to understand this and proposed a model which focused on four organizational constraints that interact with individuals' motivations to learn: (1) facilitating access to knowledge, (2) opportunity to practice and build additional skills, (3) providing effective support for the learning process, and (4) the extent to which the organization values and rewards learning.

Other research has proposed curricular models to promote learning in the workplace. Billett (2001) outlined ways in which the workplace contributes to employee learning, highlighting employee engagement in everyday work tasks, direct guidance from more experienced coworkers, and indirect guidance provided through observation or collaborations. He proposed an embedded workplace curriculum that attends to three 
levels of guided learning to aid in the development of employees. The first level develops a path that moves workers toward increasingly complex tasks, providing support and guidance along the way to mastery. The second level rests on the guidance of more experienced workers modeling, coaching, and questioning employees to deepen knowledge that could not be acquired on their own. The third level focuses on transferability by helping employees recognize the limits of their knowledge and skills, and through reflection, extend them to novel circumstances.

Clarke (2005) investigated the influence of a number of workplace environmental conditions that the literature suggests influence workplace learning outcomes, which he defined as the acquisition and utilization of procedural knowledge. He found that learning is best supported in workplaces that encourage open communication, democratic decision-making, active participation, opportunities for reflection on learning, and an appropriate level of job challenge. Although Clarke acknowledged that individual employees play a role in knowledge construction within the workplace, he emphasized that the workplace itself contributes to developing individuals" "skills or capabilities associated with 'learning how to learn"” (p. 197).

Organizational contextual factors and their mediating influence on informal learning was explored by Ellinger (2005). Four overarching themes were found to positively influence informal workplace learning: (1) organizational leadership commitment to learning, (2) learning culture permeating throughout the organization, (3) providing access to tools and resources that support learning, and (4) support of open and accessible relationships.

Finally, Eraut (2007) outlined a number of organizational factors that affect 
workplace learning. Workplaces support learning through creating a culture of support and trust, while fostering social inclusion in supportive teams. Employees are better able to learn through work when work assignments provide the right level of challenge and they have a sense of choice in how to perform their work activities. The workplace responsibilities for allocation and structuring of work, whether work is individual or collaborative in nature, expectations surrounding performance and progress, and effective feedback mechanisms all contribute to the learning potential in that environment.

\section{Social}

Social interactions also contribute to employee learning at work in a variety of ways. According to Billett (2001), learning support from social interactions can be direct, such as when individuals receive direct guidance from more experienced workers, or indirect, such as through passive observation in the workplace. Collin (2002) explored the ways in which employees conceptualize how they learn at work. Learning through cooperation and interaction with colleagues was identified as one of the primary ways in which employees learn. This included behaviors such as asking for advice, listening to others, engaging in discussions, consulting with colleagues, and learning from others' experiences.

A number of sub-themes from Ellinger's (2005) study of organizational contextual factors that influence informal learning related to social interaction. Leadership and management support employees' informal learning in a number of ways, such as pulling employees into spaces or activities that encourage their future development, coaching and mentoring, examining alternative solutions to problems alongside employees, and serving as role models. Additionally, informal learning was 
enhanced through an organization's support of developing relationships throughout the workplace and encouraging openness, knowledge-sharing, and the development of others.

Several types of social interactions that employees acknowledged contributed to their on-the-job learning were identified by Eraut (2007). These included: (1) participation in group processes, such as teams working toward a common goal, (2) working alongside others with the opportunity to observe or engage in discussion, (3) consulting with others and receiving advice from individuals within or outside the organization, (4) tackling challenging tasks with the support of others, (5) engaging in problem solving of a technical or interpersonal nature, and (6) receiving coaching, feedback, and formal or informal mentorship.

\section{Summary}

Sociocultural theories of workplace learning provide a foundation for further exploration of how learning is facilitated in the campus student employment environment. These theories emphasize the social nature of learning, the process of learning through participation, and the influence of workplace context. However, any exploration of workplace learning would be incomplete without also addressing the importance of individual motivations to learn.

On-campus student employment differs from other co-curricular experiences because it has not traditionally been explicitly centered around learning and development. Undoubtedly, learning is happening in the student employment environment, whether intentional or a by-product of engaging in a new experience. However, campus employment currently lacks an intentional and consistent unifying framework or pedagogy outlining how to effectively structure learning opportunities in the student 
employment environment. What is needed is an inquiry into the campus employment experience to understand how organizational, social and individual factors influence opportunities for learning.

\section{Conclusion}

Given its prominence in the lives of current undergraduate students, campus employment is poised to become a viable student engagement opportunity that meaningfully contributes to student learning outside the classroom. Yet, little is known about how student employees experience learning in the campus student employment environment. Sociocultural theories of workplace learning highlight individual, social, and organizational contributions to facilitating learning in the workplace environment and will form the basis of inquiry for the current study. 


\section{CHAPTER 3}

For the majority of American undergraduate students, employment is a major component of their college experience. With so many students working while attending college, it is important to understand how work experiences affect college student learning, development, and growth. The purpose of this study is to explore student employee experiences in campus jobs at a large, public Pacific Northwest university to gain a deeper understanding of the individual, social, and organizational factors that contribute to or hinder learning in the campus employment environment.

The primary research question guiding this study is: What are the individual, social, and organizational factors that influence campus employment as a learning experience for undergraduate students?

\section{Inquiry Approach}

A qualitative research approach was used to explore the research question. Qualitative research is designed to help the researcher develop an "understanding of how people make sense of their lives, delineate the process (rather than the outcome or product) of meaning-making, and describe how people interpret what they experience" (Merriam \& Tisdell, 2016, p. 15). It is an inductive process that aims to provide a richly descriptive report of the phenomenon of interest. A social abstraction, such as workplace learning, is "best understood through the experiences of the individuals whose work and lives are the stuff upon which the abstractions are built" (Seidman, 2013, p. 9). The aims of this study included developing an understanding of how learning occurs in the campus employment environment from the perspective of student employees, as opposed to empirically testing what students learn, aligning with the qualitative research method of 
inquiry.

A basic interpretive study was the specific qualitative research approach used to understand how student employees describe and make sense of their learning experiences in the campus employment environment, across the three conceptual spheres of individual, social, and organizational interactions. Rooted in constructivism, researchers engaging in basic interpretive studies attempt to understand the reality that study participants have constructed through their social interactions. As Tierney (1987) explained, "reality is not something objective or external to the participants. Instead, participant reality is defined through a process of social interchange in which perceptions are reaffirmed, modified or replaced according to their apparent congruence with the perceptions of others" (p. 64). Merriam \& Tisdell (2016) elaborated that through this design participants are able to share how they "...interpret their experiences,...construct their worlds, and...attribute [meaning] to their experiences" (p. 24).

Maxwell (2013) further clarified a strength of this approach is that it goes beyond simply describing participants' behaviors or actions to include "how the participants in your study make sense of these, and how their understanding influences their behavior" (p. 30). Additionally, Maxwell highlights the value that a qualitative approach brings to understanding how participants construct meaning within a specific context and the underlying processes that contribute to an event or action occurring. Previous research on student employment has tended to focus on the impact of work on various student success factors; however the current study aimed to understand how students conceptualize learning in the campus employment environment. The basic interpretive design allowed for exploration of the individual, social, and organizational factors that support and 
encourage undergraduate employee learning in the campus workplace.

\section{Site}

The research study was conducted at a large public university in the Pacific Northwest, henceforth referred to as PNU, with a population of about 20,000 undergraduate students. The university has a robust undergraduate student employee population of approximately 3,450, who are employed in a wide variety of position types. Student employment is not centrally supported through a formalized institution-wide curriculum or coordinating office; however, a cross-institutional grassroots movement developed over the past six years, known as Student Employment Enhancement (SEE), provides guidance to campus employers to help them make student employment experiences developmental and meaningful for student employees. The SEE movement "is a campus-wide effort that seeks to promote and improve the professional development and employment outcomes of [PNU] student employees by developing the knowledge, skills, and capacity of career staff members who supervise students" (Student Employment Enhancement, n.d., para. 1). SEE provides trainings, workshops, and resources for student supervisors to assist with effective structuring of student employment experiences, but implementation of the best practices championed by SEE is not compulsory across the institution.

Since the current study sought to explore campus employment as a co-curricular learning opportunity, the study was limited to student affairs units. At PNU, student affairs comprises four departments and mulitiple functional areas, including the student union, campus recreation, career services, the dean of students office, associated student government, parent and family programs, and centralized information technology. Two 
of the departments, the student union and campus recreation, are among the largest employers on campus.

\section{Participants}

The present study sought to understand how individual, social, and organizational factors influence undergraduate student employee learning in the campus workplace. In this study, student employee refered to currently enrolled full-time undergraduate students who were employed by the institution to work in a campus facility on a part-time basis and received hourly wages (Burnside et al., 2019). A total of 13 students employed in hourly positions within student affairs units were selected to participate in the study. The researcher sought to select student employees who could serve as quality informants. As Johnson and Rowlands (2012) explained, quality informants "have been thoroughly enculturated in the setting or community, have recent membership participation, have some provisional interest in assisting the interviewer, and have adequate time and resources to take part in the interview" (p. 105). The following section details how participants were selected.

\section{Sampling and Recruitment}

In order to select participants who could provide the greatest insight into the research question, purposeful network sampling was used (Merriam \& Tisdell, 2016). This sampling technique involved selecting participants whom the researcher thought were best able to speak to the central phenomenon of interest. The following criteria guided the selection of quality informants for this study. First, since the study focused on the experience of undergraduate student employees, all participants had to be enrolled full-time undergraduate students at PNU. Second, as the study focused on employment 
within student affairs units, students had to be employed in one of the units within the PNU Division of Student Life. Finally, in order to ensure they had sufficient work experience to reflect upon, participants must have been employed within the same department on campus for at least three terms.

The researcher will obtained referrals of student employees who meet the participation criteria from student supervisors within student affairs units to drive recruitment. As a current employee at PNU, the researcher leveraged her network and established relationships to encourage responses. An initial email was sent to all 39 supervisors of student employees across the division of student affairs detailing the purpose of the study and the criteria for participation and asking for help identifying qualified student employees (Appendix A). Once a list of potential participants was obtained from student supervisors, the researcher emailed the identified individuals directly, with a message including the purpose of the study, criteria for participation, and expected commitment (Appendix B). Individuals interested in participating were directed to fill out a Qualtrics form (Appendix C). After reviewing responses, the researcher sent one follow-up email to increase the pool from which to select participants.

\section{Introduction to Participants}

To represent a breadth of perspectives on workplace learning, participants were selected for maximum variability. Lincoln and Guba (1985) proposed that maximum variation sampling allows the researcher to pull from a diverse array of perspectives to discover commonalities in the experiences of participants. Patton (1990) also wrote about the significance of finding commonality and shared themes amongst the variety of participants. The primary variance consideration was ensuring that selected participants 
represented a variety of position types and departments within student affairs units. The researcher also sought to select participants who represented a breadth of diversity in gender, race, and ethnicity.

Thirteen total participants were selected and interviewed for the study. Participants represented a variety of backgrounds and a diverse array of experiences. Due to the small sample size, sensitive and potentially identifying information is summarized here, while Table 1 provides a brief overview of each participant's work profile. The gender breakdown of the group included seven females, five males, and one participant who identified as gender fluid. Age of participants ranged from $19-28$, with two participants falling outside the traditional-aged undergraduate student range. Nine participants were white and the other four were Black or Mixed race. Four students were the first in their families to attend college, and eight were from families that had lower socioeconoic status. Participants were assigned pseudonyms to preserve their anonymity. Table 1 provides a summary of demographics, which were self-reported by participants.

Table 1

Introduction to participants

\begin{tabular}{|c|c|c|c|c|}
\hline Pseudonym & Department & Job Type & $\begin{array}{c}\text { Terms } \\
\text { Worked }\end{array}$ & $\begin{array}{l}\text { Year in } \\
\text { College }\end{array}$ \\
\hline Jonathan & Student Government & Administrative Support & 3 & 2nd \\
\hline Drew & Dean of Students & Student Support Services & 4 & 4 th \\
\hline Mike & Information Technology & Technology Consultant & 12 & 4th \\
\hline Tyler & Campus Recreation & $\begin{array}{c}\text { Programming Coordinator } \\
\text { \& Lifeguarding }\end{array}$ & 8 & 4 th \\
\hline
\end{tabular}




\begin{tabular}{|c|c|c|c|c|}
\hline Bo & Student Union & Building Services & 3 & 2nd \\
\hline Amanda & Campus Recreation & $\begin{array}{c}\text { Operations \& } \\
\text { Personal Training }\end{array}$ & 7 & 3rd \\
\hline Kelly & Dean of Students & Student Support Services & 6 & 4 th \\
\hline Sarah & $\begin{array}{c}\text { Parent \& Family } \\
\text { Programs }\end{array}$ & Administrative Support & 7 & 4 th \\
\hline Shannon & Student Union & Programming Coordinator & 3 & 4 th \\
\hline Kyle & Student Union & Program Support & 3 & 5 th \\
\hline Jackie & Student Government & Administrative Support & 7 & 4 th \\
\hline Angie & Student Union & Programming Coordinator & 4 & 2nd \\
\hline Heather & Career Center & Administrative Assistant & 6 & 4th \\
\hline
\end{tabular}

Working was a big part of college life for most of the student employees interviewed. All but two of the participants worked prior to coming to college, and the two that did not work prior to starting college were heavily involved in high school clubs and community service. The number of hours worked per week in their campus positions ranged from 9 to the university-allowed maximum of 25 during the term and 40 off-term. Nine of the study participants worked in multiple positions at the time of the interview.

\section{Ethical Considerations}

The following elements of the study were implemented to ensure that participants had a complete understanding of the research purpose, what would be done to protect their confidentiality throughout the process, and their rights as participants. Prior to the study commencing, the researcher obtained approval from the Portland State University Institutional Research Board (IRB). The researcher also submitted for IRB approval at 
the other institution, which defered to Portland State for review.

When participants arrived for their interview, they received a written description of the study purpose and informed consent form (Appendix D). The researcher briefly described its contents, encouraged participants to read through it in its entirety, and answered any questions prior to their signing.

In preparing to work with research participants, the researcher considered how to best negotiate that relationship, including building rapport, making sure the purpose and goals of the research were clear, explaining how data would be used, and obtaining informed consent in order to ensure ethical data collection (Maxwell, 2013). Additionally, considerations were made on how to best protect the confidentiality of participants. Interviews were recorded using a digital audio recorder to accurately maintain a verbatim record of each participant's words. Recorded interviews were then transferred to the researcher's computer and saved in a password-protected file for the duration of the study. Participants were given a pseudonym that was used throughout data analysis and within this final report.

\section{Data Collection Process}

In qualitative research, the researcher is the primary instrument, bearing sole responsibility for determining what information constitutes study data (Merriam \& Tisdell, 2016). Data collection for this basic interpretive qualitative study comprised a 90-minute interview with each study participant, which was recorded on a Sony Digital Voice Recorder and on the researcher's cell phone using the Otter AI application. All interviews were conducted between August and October, 2019 and each interview lasted approximately 90 minutes. 


\section{Participant Interviews}

Interview data was collected using in-depth, semi-structured interviews with study participants. According to Johnson \& Rowlands (2012), in-depth interviewing "is best suited to research questions of the descriptive or exploratory type" (p. 101). In-depth interviews allowed the researcher the opportunity to uncover the typically internalized conscious reflections or unconscious thoughts through participants' accounts of their experience. The semi-structured interview format provided a thematic guide for the researcher while still allowing flexibility for pursuing new avenues as they arose throughout the interview process (Merriam \& Tisdell, 2016).

Seidman (2013) proposed that the researcher preliminarily reviews enough of the literature on a topic to inform their inquiry. The interview protocol was informed by the literature reviewed in Chapter 2 (Appendix E). The study pulled from multiple conceptual angles, including the student engagement and co-curricular learning constructs, which are central components of student affairs, and individual, social, and organizational factors that inform sociocultural workplace learning theories. Questions were mapped into broad categories of individual epistemologies, student engagement, and sociocultural workplace learning theories to guide the questions. A selection of questions are displayed in Table 2, along with their purpose and mapped category.

\section{Table 2}

Select Interview Protocol Questions

\begin{tabular}{|l|l|l|}
\hline Question(s) & Purpose & $\begin{array}{l}\text { Related } \\
\text { Theory/Construct }\end{array}$ \\
\hline What are your reasons for & Rapport-building/demographics & N/A \\
\hline
\end{tabular}




\begin{tabular}{|c|c|c|}
\hline $\begin{array}{l}\text { working while attending } \\
\text { college? }\end{array}$ & & \\
\hline $\begin{array}{l}\text { How would you describe the } \\
\text { knowledge and skill set a } \\
\text { person would need to } \\
\text { successfully do your job? } \\
\text { How have you personally } \\
\text { developed these skills? }\end{array}$ & $\begin{array}{l}\text { Understand how employees } \\
\text { conceptualize learning and their } \\
\text { perceptions of skill development }\end{array}$ & $\begin{array}{l}\text { Sociocultural } \\
\text { Workplace Learning } \\
\text { Theories }\end{array}$ \\
\hline $\begin{array}{l}\text { In which ways have your } \\
\text { personal background and } \\
\text { previous educational } \\
\text { experiences helped prepare } \\
\text { you to perform your job } \\
\text { duties in this position? }\end{array}$ & $\begin{array}{l}\text { Understand how personal } \\
\text { backgrounds and prior educational } \\
\text { experiences influence current work }\end{array}$ & $\begin{array}{l}\text { Individual } \\
\text { Epistemologies }\end{array}$ \\
\hline $\begin{array}{l}\text { In which ways do you work } \\
\text { with other people in your job? } \\
\text { What, if anything, have you } \\
\text { learned through these } \\
\text { interactions? }\end{array}$ & $\begin{array}{l}\text { Understand the social dynamics at } \\
\text { work and how the worker describes } \\
\text { their influence on learning. }\end{array}$ & Student Engagement \\
\hline $\begin{array}{l}\text { What are some things that } \\
\text { you have learned through } \\
\text { working in this job? Who or } \\
\text { what specifically contributed } \\
\text { to that learning? In which } \\
\text { ways do you think your } \\
\text { workplace supports your } \\
\text { learning? }\end{array}$ & $\begin{array}{l}\text { Understand what has been learned } \\
\text { and to what or whom the student } \\
\text { attributes the learning }\end{array}$ & $\begin{array}{l}\text { Sociocultural } \\
\text { Workplace Learning } \\
\text { Theories }\end{array}$ \\
\hline $\begin{array}{l}\text { When you think about what } \\
\text { you have learned in your } \\
\text { academic classes, in which } \\
\text { ways have you been able to } \\
\text { apply that knowledge into } \\
\text { your work? }\end{array}$ & $\begin{array}{l}\text { Understand the ways in which } \\
\text { learning is extended beyond the } \\
\text { classroom }\end{array}$ & Student Engagement \\
\hline
\end{tabular}

The questions addressing individual epistemologies of students allowed participants to describe their personal histories and background experiences as they relate to engagement at work (Fuller \& Unwin, 2004). Additionally, these questions helped 
expose participants' motivations for engaging in work and learning through work (Billett, 2001). Participants also identified their perceived value of the work experience, opportunities for growth, and alignment with future goals in answering these questions (Collin, 2002).

Questions addressing the student engagement construct allowed for further exploration of how campus employment extends learning beyond the classroom (Kuh, 2008). Participants reflected how they have applied, transferred, and integrated learning across contexts (Hansen \& Hoag, 2018). Finally, these questions gave them an opportunity to consider their personal growth that has taken place through campus employment and explore the ways in which the department or people within it supported that growth and development (Kuh, 2009).

Questions categorized under sociocultural workplace learning theories encouraged participants to share their insights on the social and organizational dynamics of their workplace. Participants described their workplace interactions with others (Eraut, 2004), their perceptions on how the workplace supports their learning (Billett, 2001; Ashton, 2004), and how they solved novel challenges (Clarke, 2005).

Two test interviews were conducted with student employees who were not included in the study. The test interviews served three purposes: (1) determine if the questions elicit the types of responses that will be useful for informing the research questions, (2) determine if any of the questions are confusing to the interviewee, and (3) allow the researcher an opportunity to hone her interviewing skills. After the test interviews, the researcher made minor adjustments to the final interview protocol. Weiss (1994) said "the investigator will develop insights, speculations, and small- 
scale theories beginning with the first pilot interview or before" (p. 151). These thoughts were collected into a researcher journal that was added to throughout the data collection and analysis process. The researcher maintained these memos to record their initial thoughts and hunches, which later helped with data analysis.

\section{Data Analysis}

Maxwell (2013) asserted that data analysis should not be an afterthought to the research project, but rather must be a well thought-out part of the full design. Saldaña (2016) emphasized that organization is an essential component of qualitative data analysis. All incoming data from this study, including audio recordings and researcher memos were named, dated, and stored in password-protected digital files. They were also backed up in a password-protected cloud-based storage.

Interview transcription was performed by the interviewer in order to increase familiarity with the data. Initial data analysis commenced upon transcribing the final interview. Data were coded by the researcher using initial and a priori coding methods (Saldaña, 2016). Initial coding, also referred to as open coding, was used sparingly, but allowed the researcher the flexibility to remain open to theoretical possibilities while assigning tentative labels to the data. A priori coding was generated from a set of codes pulled from the literature relating to the central concepts of individual, social, and organizational factors that influence workplace learning, as outlined in Table 3.

\section{Table 3}

\section{A Priori Codes}

\begin{tabular}{|l|l|l|}
\hline Individual & Social & Organizational \\
\hline
\end{tabular}




\begin{tabular}{|l|l|l|}
\hline Confidence & Guidance & Worker Autonomy \\
Self-Efficacy & Observation & Novel Experiences \\
Internal Motivation & Engaging in Dialogue & Feedback Mechanisms \\
Prior Knowledge & Learning from Other & Reflective Opportunities \\
Background & People's Experiences & Learning Culture \\
Disposition & Mentoring/Coaching & Increasing Complexity \\
Active Participation & Role Modeling & Transferability \\
Seeking Guidance & Knowledge-Sharing & Challenge \\
Locating Resources & Participation in Group & Committed Leadership \\
Taking Risks & Processes & Work Allocation \\
& Working Alongside Others & \\
\hline
\end{tabular}

As the researcher worked through initial data analysis, they continued to reflect their deep thinking through researcher memos (Maxwell, 2013). Saldaña (2016) stressed the importance of maintaining written memos throughout the research process, calling them "a question-raising, puzzle-piecing, connection-making, strategy-building, problemsolving, answer-generating, rising-above-the-data heuristic" (p. 44). Using the generated codes as a launching point, researcher memos provided an opportunity for reflection and on connections between disparate coded data segments.

After the data was coded into distinct units, it was rearranged "into categories that facilitate comparison between things in the same category and that aid in the development of theoretical concepts" (Maxwell, 2013, p. 107). Categorizations were kept broad and varied at the outset. However, as data collection and analysis continued, category construction turned from an inductive process into a primarily deductive process in which the data was checked against the categories the researcher constructed (Merriam \& Tisdell, 2016). Weiss (1996) referred to this type of analysis as issue-focused analysis, which links themes and categories across respondents to describe what has been learned 
about the research topic. Once the data was sorted into categories, the researcher started interpreting the data, a process that Weiss (1996) refers to as "local integration" (p. 158). The working hypotheses that developed at this point were tested against the data and either integrated into the final interpretation or discarded if they did not hold up to scrutiny.

The final step in analysis involved writing the report. "Researchers seeking to describe an experience or event select what they will describe and, in the process of featuring certain aspects of it, begin to transform that experience or event" (Sandelowski, 2000, p. 335). The researcher was ethically responsible for ensuring that they conveyed the information presented in a way that accurately represented the participants views and the meaning they attributed to the phenomenon under study. Validity in basic descriptive studies relies on accurate description and interpretation of the data.

Throughout the analytic process, steps were taken to address validity threats. In qualitative research, validity refers to "the correctness or credibility of a description, conclusion, explanation, interpretation, or other sort of account" (Maxwell, 2013, p. 122). As suggested by Patton (2015), multiple qualitative data sources, including interview transcripts, notes, and researcher memos, were scrutinized against each other to look for consistencies in patterns or places of divergence. After initial conclusions were written up, a peer was asked to review the findings and provide feedback on areas perceived as problematic.

\section{Researcher Positionality}

Prior to conducting this study, it was important to take time to examine my interest in pursuing research on the topic of campus employment and acknowledge the 
aspects of my own identity, background, and assumptions that might influence the research process. As Patton (2015) states, "the trustworthiness of the data is tied directly to the trustworthiness of those who collect and analyze the data - and their demonstrated competence" (p. 706).

As an undergraduate student, I was employed by the Campus Recreation department for two summers as a youth camp counselor. When I reflect upon that experience, I am able to recount numerous skills that I developed or honed in the two summers I held that position. Spending day after day getting and keeping the attention of large groups of children helped develop me into a confident public speaker. Holding responsibility for the planning and implementation of daily activities taught me the importance of having a structure, but not holding too rigidly to it when the best laid plans did not play out as expected. Speaking with a parent whose child had a rough day taught me how to have empathy in navigating difficult conversations. Not only did my employment experience help with my skill development, but it also set the course for my professional career within the field of Campus Recreation.

Throughout my eleven-year career in Campus Recreation, I have supervised hundreds of student employees in a variety of roles, including youth camp counselors, program assistants, instructors, and program supervisors. My supervision practice has always been grounded in student development theory and firmly rooted in the belief that student employment can be both developmental and meaningful for student employees. Although I no longer directly supervise student employees, I continue to share that philosophy as the chair of my department's Student Development Team, comprising all the employees who hold student supervision responsibilities. 
As a highly reflective learner, I spent the first few years in my supervision practice observing student employees, asking questions, evaluating, and seeking guidance from more experienced supervisors. As I engaged with student employees, I heard stories from students who felt that their work experience was the most meaningful thing they did in college. Many felt that the lessons and skills they learned through the workplace were relevant, tangible, and transferable. These types of comments became motivating for me to hone my own supervision practice and structure the student employment opportunities I facilitated to provide maximum benefit for even more student employees. The conversations I have had with former employees in subsequent years have helped solidify my belief that campus employment has the potential to be a highly influential factor in undergraduate student learning and development.

For the past four years, my interest in and depth of knowledge related to student employment has grown through my engagement in the Student Employment Enhancement (SEE) efforts on campus. Throughout that time, I have contributed to SEE through facilitating workshops on student supervision, developing a guiding document on best practices in student supervision, providing one-on-one consultations with student supervisors, and establishing the SEE Summit, an annual conference-style professional development opportunity. Currently I chair the SEE Steering Committee, providing guidance on the future direction of the campus-wide effort. My work with SEE has exposed me to the wide variety of student employment experiences available on campus and helped me recognize that some employment experiences seem to provide greater benefit to student employees than others. This has led me to want to explore more deeply what are the common elements that contribute to student employee learning in the 
campus employment environment so that I can share the findings with my colleagues in an effort to elevate all campus employment experiences.

"Today, there are many researchers who use their investigations and interviews to explore phenomena about which they have prior or current member-based knowledge" (Johnson \& Rowlands, 2012, p. 103). Although my prior knowledge and involvement with student employment on multiple levels provides assurance of my competence to engage in the study, it also offers the potential pitfalls of introducing researcher bias. For this reason, it is important to self-reflect on my own positionality as it relates to the research topic and potential biases both before and throughout the research process.

I recognize that my experiences have shaped my perspectives on student employment and could present a bias toward interpreting the data in a way that supports learning as inherent in the campus workplace. To address this bias, I will analyze the data with an open mind and test it against my preconceptions before drawing conclusions. The multiple data source scrutiny and peer review strategies previously outlined will also be used to keep researcher bias in check.

\section{Limitations}

The research plan was carefully considered and structured to address as many limitations as possible; nevertheless, certain limitations to the research project remain. First, as the study was conducted with a relatively small number of students at a single institution, the experiences shared by students may not be universally applied or generalizable to student employees at all institutions. Additionally, the size of the group was insufficient to uniquely identify shared experiences of students across various demographics. This is consistent with a limitation found within most qualitative studies 
(Merriam \& Tisdell, 2016). Second, the participant recruitment strategy may have posed a limitation. Although the outreach materials asked for a generalized referral of any student employees who meet the study criteria, relying on supervisors for referrals might mean that only standout student employees got put forth. Third, since student employees were pulled from multiple types of positions and departments on campus, they had quite distinct campus employment experiences, which posed a challenge in grouping and cataloguing shared themes. Conversely, since student employees were only selected from student affairs units, they may reflect a more learning and development-centered employment experience than their peers in non-student affairs units would, due to the assumed role student affairs plays in attending to outside-of-class learning. Finally, in this qualitative research study, the researcher served as the primary instrument in data analysis, which could introduce bias or misrepresentation of participant interviews and ultimately untrustworthy findings. To minimize this limitation, researcher positionality in relation to the study has been examined and explicitly laid out. Throughout the study, alternative explanations were tested against researcher conclusions to check for potential biases.

\section{Chapter Summary}

The purpose of this study was to develop a better understanding of how learning is facilitated in the campus employment environment. The study's conceptual framework was informed by student engagement and co-curricular learning, as well as sociocultural theories of workplace learning, which will allow for the exploration of the individual, social, and organizational factors that support or hinder student employee learning.

The research took the form of a basic interpretive study, in which current student 
employees described their learning experiences through campus employment. Participants were made up of 13 student employees selected from student affairs units at a large, public Pacific Northwest university. Data was collected using in-depth, semistructured interviews with study participants. The interview protocol was informed by the three conceptual areas of student engagement, co-curricular learning, and sociocultural theories of workplace learning. Data was coded using open and a priori coding methods, analyzed into categories, and interpreted by the researcher to produce the final report.

As universities continue to embrace institution-wide support for transformative student learning, it is important to understand how all university-sponsored programs can effectively contribute to that effort. Campus employment is one of the larger touchstones on campus for students, yet has infrequently met its potential as a means of engaging students in educationally purposeful activities. Previous research on student employment has focused on its liabilities; however, to best serve the growing majority of students who rely on employment for financial support, it is imperative to shift the discourse toward discovering how student employment might enhance the college experience. The next chapter outlines study results and analysis. 


\section{CHAPTER 4}

This study explored the factors that facilitate campus employment as a learning experience for undergraduate student employees. With the increase in work intensity and frequency for contemporary college students, it is especially important to understand how campus employment can be effectively structured to engage students in educationally purposeful activities. This has implications for student success metrics, including retention and graduation rates, as well as post-college career readiness. The data substantiate that, when done well, campus employment provided student employees a number of benefits and co-curricular learning opportunities.

This chapter highlights research findings and emergent themes, starting with a review of why students work while attending college and the learning outcomes associated with participation in student employment. The chapter then turns to an exploration of when learning is present in the campus employment environment, grounded in Bandura's social cognitive learning theory and reciprocal determinism between personal, behavioral, and environmental factors. Finally, the chapter concludes by considering the mediating factors that influenced the intensity with which student employees experienced campus employment as a learning opportunity.

\section{Working Undergraduate Students}

This research focused on learning experiences within campus employment, and participants were recruited based on their perceived ability to provide insightful information into the research topic. Participants in this research study comprised undergraduate students who were employed on campus between three to twelve terms. All of the participants worked hourly positions within student affairs units in a variety of 
position types.

\section{Work Intensity and Frequency}

Campus employment was a time-consuming outside-of-class experience, with the student employees interviewed working anywhere from 9 to 25 hours per week during academic terms in their campus positions and a majority holding more than one job simultaneously. Though working during college is a near-universal experience for undergraduate students, older students, students with lower socioeconomic status, first generation students, and students from traditionally underrepresented minority groups are even more likely than their counterparts to carry heavy workloads (Choi, 2018; King, 2006; McCormick et al., 2010). Ten of the thirteen participants in this research demographically aligned with at least one of those categories. Campus employment served as a primary engagement and co-curricular learning experience for the student employees in this study who came from underserved backgrounds, providing a catalyst for personal growth and professional development.

The research on the impact of hours worked on academic and cognitive outcomes is inconsistent and varied (McCormick et al., 2010). Given the prominence of campus employment in the lives of undergraduate students, it is vital to understand how the experience can be effectively facilitated as a learning experience. The students involved in this study overwhelmingly perceived that their involvement in campus employment constituted a learning experience. In describing what her work meant to her, Amanda shared, "They've taught me a lot of things I never could have learned in a classroom." As Suskie (2015) pointed out, the changing and diversified backgrounds and experiences of undergraduate students necessitates an evolution in co-curricular learning experiences, 
and campus employment provided one such experience for students involved in this study.

\section{Why Work}

Prior research has shown that the primary reason undergraduate students cite for working during college is to cover the expense of tuition, fees, and incidentals (Cheng \& Alcántara, 2007). Among the student employees who participated in this study, earning money to support or supplement their educational expenses was also the most commonly held reason, mentioned by every participant. As Drew shared, "I don't have a choice but to work. My family can't financially support me...I'm here on Pell Grant, [but] even my tuition refunds aren't enough to cover all my needs, so I have to work." The second most common reason given was building their resume and gaining relevant and transferable work experience. Multiple student employees also commented on how the structure of student employment helped them establish routine to better manage their time as a student, such as Angie, who felt that her campus position helped keep her "balanced and motivated." Other reasons students shared for working during college included molding to societal expectations, building their resume, having an opportunity to apply classroom learning in a real world environment, and better integrating with the campus community.

\section{Learning Outcomes}

When considering campus employment as a learning experience, it is natural to wonder what, exactly, are students learning in that environment. An increasing focus in higher education is the development of competency-based outcomes. Competencies cannot be acquired, as in the knowledge that is collected through classroom experiences, but rather must be developed (Illeris, 2011). Keeling (2004) took this idea even farther, 
arguing that the learning experiences in college must be transformational, aiding students in their identity development while teaching them to be lifelong learners who are able to adapt to new environments and integrate various sources of knowledge. Learning Reconsidered outlined seven outcomes of a transformative liberal education, including cognitive complexity; knowledge acquisition, integration, and application; humanitarianism; civic engagement; interpersonal and intrapersonal competence; practical competence; and persistence and academic achievement. Campus employment is uniquely poised to support competency development in each of these domains, and in conjunction with academic classes, guide students in their academic and professional identity development.

Campus employment is also uniquely positioned to provide student employees with career readiness skills. The National Association of Colleges and Employers (NACE) (n.d.) has outlined eight competencies that align with student readiness for a successful transition into the workplace beyond college. These competencies include critical thinking and problem solving, oral and written communications, teamwork and collaboration, digital technology, leadership, professionalism and work ethic, career management, and global and intercultural fluency. The student employees in this research outlined a number of ways in which they perceived their campus employment experience was equipping them with the requisite competencies to successfully launch into the workforce. Participants in this study indicated that through campus employment they developed a number of the competencies that align with institutional and employer goals in the areas of interpersonal oral and written communication, professionalism, critical thinking, problem solving, leadership, teamwork, and collaboration. 


\section{Interpersonal Communication}

Several student employees reported developing elevated levels of interpersonal communication skills through their campus positions. All of the student employees interviewed interacted with other people in their workplace, which required that they enhance their communication skill set through situations such as learning to navigate office politics, working through difficult conversations, giving and receiving feedback, and interacting with others who had different opinions and work habits. Students who worked in customer-facing positions also learned to effectively communicate with individuals outside their organization.

Jonathan provided an example of the development of external communication skills. He felt that he developed this skill set primarily through early miscommunications in interactions with students who visited his workplace. He recognized that the students coming in might have different levels of understanding related to the technical aspects of their inquiry and learned to adjust his communication style to meet their needs. To achieve that, he would 'take a second to listen to what they're saying and figure out what level they're on, from very basic to advanced," rather than making assumptions about what they already knew. From there, he could tailor his communication to specifically address their needs.

Drew and Kelly's positions both required a tremendous amount of communication with external constituents, including coordination of recruitment efforts. Both student employees expressed gaining a deeper understanding of effective communication techniques through participation in these processes and learning through previous failures and successes. 


\section{Professionalism}

The professionalism competency relates to students' abilities to hold personal accountability, manage their workload, and work productively with others. Mike, Heather, and Bo expressed that they gained competence in navigating bureaucracy in the workplace. As Mike noted, navigating structures in a big organization is "way harder than it sounds," even for people who are technical experts in their jobs. He considered it "useful insight...because that is not exactly something that most people take classes in, like how to deal with bureaucracy." Through watching other people successfully, or in some cases unsuccessfully, navigate bureaucratic systems in his workplace and also operating in those systems himself, he gained competencies that will transfer over into future jobs.

Amanda also touched on aspects of developing professionalism, including becoming more disciplined in her work ethic and developing personal accountability. Within her workplace, there was a lot of trust put in student employees to set their own schedule and appropriately use and report their time. Throughout her employment, she enhanced her time management at work and learned to more effectively manage her workload and prioritize her time.

Sarah similarly felt that her time spent in campus employment enhanced her professionalism skills. As she described, "I did have some secretarial background, so like answering phone calls, emails messages, things like that. But, this is definitely hyperfocused, and there's just so much more to attend to." Sarah internalized what it meant to be a representative of the university in her position and felt personal accountability to doing a good job, adjusting her behaviors accordingly. 


\section{Critical Thinking and Problem Solving}

Student employees gained competence in problem solving and critical thinking skills, demonstrated through their descriptions of analyzing problems, effectively using their resources, and making sound decisions. Several student employees were surprised to find that their actual job duties were more complicated than their position descriptions led them to believe. As Jonathan described:

A lot of my work has surprised me because it's not as black and white as I think I initially saw it on the application. They said it was going to be customer service, and it is customer service, but it's not just a ton of transactions where you're sitting down, getting asked three questions, giving them an answer, and then the next person comes up.

The dynamic work environment and spectrum of questions that came up in that context required that Jonathan engage his critical thinking skills on a regular basis to provide customer service and reach satisfactory resolutions to customer issues.

The nature of Mike's position required him to consistently engage in problem solving daily. He often encountered challenges that did not have a straightforward solution and would rely on his own previous experience, historical records, and the collective knowledge of others in the workplace to draw out novel solutions. Drew also engaged critical thinking skills in their work by staying up to date on current events and politics and integrating that into the interactions they had with the students using their support services.

Tyler similarly described a dynamic nature to his workplace that required active engagement of problem-solving skills. As he described, "It's like playing whack a mole. You solve one problem and another problem pops up." He felt prepared to adequately 
address problems from developing his "toolbox" of techniques over time and being willing to try new solutions, even if there was a risk of failure.

\section{Leadership}

Leadership skills were developed as student employees took on both formal and informal leadership roles. This competency was demonstrated through student employees effectively developing, motivating, and coordinating others. Some student employees actively sought opportunities to develop this competency, while others were pushed into leadership roles based on seniority.

Tyler sought out leadership opportunities through campus employment, eventually advancing to lead positions. In those roles, he carried supervisory responsibilities that entailed oversight of his peers and providing effective guidance and management. He developed this skill set through observing his own supervisors and reflecting on their actions, attending conferences and workshops, and seeking feedback from others.

Amanda also held a positional leadership role and described the development of her skill set to fulfill the required responsibilities. As a manager, she felt that it was important to both provide support for her team while still holding them accountable to job expectations. She explained the evolution she went through of finding a balance between holding the line on expectations while still remaining approachable and fair, which included actively reflecting on her role as a leader and how she could improve in her leadership capabilities.

Over the course of his employment, Mike transitioned into a leadership role, providing guidance, informal mentorship, and training to newer student employees. 
Though his positional title did not in the multiple years with his department, the cumulative knowledge and experience he gained allowed him to naturally fall into that leadership role among his peers.

\section{Teamwork and Collaboration}

Several student employees worked with others toward accomplishing larger goals and learned how to effectively work as a member of a team through that process. Kelly and Drew coordinated large-scale events that involved multiple stakeholders in their jobs. While they both indicated learning to trust their own intuition and skills to guide the process, they also developed increasing levels of trust as they collaborated with others. Kelly also expressed developing deeper levels of empathy for the other student employees she supervised, putting herself in their shoes and recognizing that encouraging mental self-care was an important part of accomplishing their collective goals.

In the course of his work, Kyle leveraged the strengths of others to fill in gaps in his own skill set as he planned and ran events. Not only would he connect with them to vet ideas, but he also relied on their expertise in scheduling and marketing events. $\mathrm{He}$ recognized his own strengths and the ways in which he could leverage the strengths of others to accomplish collective goals.

\section{Summary}

Through their campus positions, undergraduate students in this study indicated development in numerous competencies related to the goals of a liberal education and career readiness. Though previous research has had mixed results on the effects of student employment on students success, the evidence provided makes a case that campus employment does provide an educationally purposeful experience for student 
employees. Further, the students involved in this study largely represent populations that have been underserved by higher education. Campus employment provides an accessible engagement opportunity that both helps support students financially while also providing a developmental experience. The next section considers the factors that influence learning in the campus workplace.

\section{Factors That Influence Learning}

The following sections turn to answering the question of how learning is influenced within campus employment experiences. Sociocultural theories of workplace learning attempt to explain the interactive factors which support learning in that environment, considering the workplace as a social context in which individual behaviors are shaped by both the environment and social interactions. Results from this research suggested that within the campus workplace, what student employees do and how they think is influenced by the workplace environment and the people within it, and vice versa. As these three factors interacted, student employees had enhanced learning and development experiences, which collectively contributed to a heightened sense of academic and professional identity. If campus employment is to be realized as an opportunity to help student employees develop their academic and professional identity, it is important to understand the factors that influence the campus workplace as a learning environment. The following sections analyze the personal, interpersonal, and environmental factors that student employees interviewed indicated facilitated learning through campus employment, followed by a discussion of the reciprocal interactions and mediating factors that influenced the intensity of the learning effectiveness.

\section{Personal}


Campus workplaces are filled with individuals who each carry unique identities, backgrounds, dispositions, attitudes, and experiences, all of which influence their capacity and motivation to learn in that setting. Fuller and Unwin (2011) asserted that life history and prior experiences influence how individuals behave and their capacity to learn within the workplace. In analyzing the data from this research, it was evident that personal backgrounds, disposition, and motivations of student employees influenced the degree to which they engaged in workplace learning. Study participants described a variety of ways in which they played an active role in influencing learning behaviors within their workplace, whether consciously or subconsciously, through their personal identities, motivation, learning orientation, and resilience.

\section{Identity}

Although the workplace learning literature has addressed background, prior experiences, and knowledge as influencing factors in how the workplace is experienced as a learning environment (Fuller \& Unwin, 2011), the influence of identity has not been explicitly considered. However, when examining the influence of personal factors in the campus workplace, identity emerged as an important personal factor. The literature has established that college is a time where students explore their identity and develop a greater sense of self. The identity development framework first proposed by Chickering and later refined by Chickering and Reisser (1993) designated seven vectors to explain the influence of the college environment emotionally, physically, socially, and intellectually in the formation of identity. Identity is important because it influences a student's conception of self as well as the perception others have of them. Students reflected a number of identities that influenced their engagement in work, including race, 
sexual orientation, and socioeconomic status, among others. The following is an exploration of select identities and intersections of multiple identities that were reported by students as influencing the ways in which they interacted with others and approached the work.

Race. Racial identity was a salient personal factor for the students of color who participated in the study. It influenced the type of position these students worked in, the ways in which they approached the work, and their interaction with coworkers. All of the students of color in this study were the sole employees in their workplace who held identity-based support positions. Workplace experiences provided an opportunity for students of color to explore their identity in relation to the world. As one student described, "Especially at this [predominantly white] school, it's helping me navigate my own identity and my own path as a minority student." Through interactions with other students of color outside their workplace, another student learned their stories and rationale for not participating in certain activities, which caused them to examine their own privilege within their family and upbringing, as well as enact change in their workplace practices.

Carrying the sole burden for educating colleagues on social justice issues was an unexpected element of campus employment experiences for several students who were racial minorities. Students not only were supporting other students, but were also typically relied on as educators for career staff members within their workplace. As one student described, "My coworkers and boss probably recognize that I'm the one always talking about social justice and racial equality... Sometimes it can be discouraging to be the only one talking about it, but also, that's who I am." Carrying this responsibility in 
working with identity-based support services also carried high burn out potential.

As one of the few people of color in her workplace, another student employee would often initiate dialogue with other employees to increase their collective cultural competence:

Good intentions aren't necessarily enough. Everyone I work with is amazing and inclusive and have the most sincere intentions, but don't necessarily understand different experiences... When you get that monoculture, you kind of become blind to how other people's experiences are, even if you can intellectually understand them. Even if you can intellectually understand racism is bad, until you actually talk to somebody who has experienced it, and they explain to you their anxieties, that won't resonate as well.

She often held the role of educating others in her workplace in these conversations, with information she had gleaned both from personal experience and from engaging with the diverse populations she was recruiting for her programs. Through those conversations, she also gained a fuller understanding of barriers to participation for people of color that differed from her own background and experiences, despite their shared racial identity.

Sexual Orientation. One student identified sexual orientation as a salient identity that influenced their work. The student worked in an identity-based support service and was surrounded by other people who were part of the LGBTQIA+ community. They expressed their perceived importance of sharing an identity with the students they served:

I feel like you almost have to [share this identity] or be a very good ally to do work like this, because you have to be very knowledgeable - and outside of your own identity too. And even still, it gets hard to keep up...[Having] a certain attitude and knowledge about the community is the most important thing, because everything else can be trained. You just really have to have that like hard work and dedication to your community, because if you don't have it, you're probably going to get burnt out.

Recognizing that there were numerous identities within the LGBTQIA+ community, this student was motivated to learn more about the experiences of other students to provide 
adequate support.

Socioeconomic Status. A student's socioeconomic status influenced which positions they worked in and their expectations for the job. Students whose families were lower socioeconomic status than their peers were primarily influenced to pursue work during college due to their need to supplement the cost of college. One student's comments summed it up for the group, stating his primary expectation was "to make money" when he took his position. In comparison to their peers, these students were less likely to select a position because it matched up with their learning goals or future career ambitions and more likely to take whatever campus position was available at the time. Most of these students also entered their campus positions with low expectations for what they would get out of their jobs.

Multiple Dimensions of Identity. A conceptual model of multiple dimensions of identity was put forth by Jones and McEwen (2000), which described the ways in which dimensions of identity intersect to form an individual's core identity. Certain dimensions of identity may be more salient than others at any given time and context, and the campus workplace provides one such context. This research supports and extends Jones and McEwen's research in recognizing the dynamic and complex ways in which multiple dimensions of students' identities influenced how they engaged at work. For instance, one participant was a former foster youth, adult adoptee, and first-generation and nontraditional aged student. The intersection of these multiple identities influenced the type of position they selected, their expectations for campus employment, interactions they had with peers and supervisors, and motivation to fully engage in the work.

Intersectionality "explicitly situates identity as multiple and layered and existing 
at once within systems of both oppression and privilege" (Jones, 2009, p. 289). It is intricately connected to the power and privilege inherent within sociocultural and historical contexts. How student employees' identities crossed at the intersections related to the systemic advantage or disadvantage they experienced through campus employment and within the larger higher education context. One student employee, who identified as Black, female, low socio-economic status, and first-generation, shared about how intersectionality influenced how she viewed campus employment and her role in the university:

I grew up with a single parent who did amazing. And you know, for the most part, we were fed, we are happy. So, I think coming from not a typical, stable background gave me a lot of insight into how I can prevent that from being my kid's situation. And then being in restorative justice [classes] gave me a big passion for social justice. So, I think I see disparities and I see inequalities in general and on campus that someone who isn't so passionate about or hasn't had the experiences I have probably wouldn't see so much. So, I think it's so important to have more diversity in faculty, staff, and students.

The complex identity of this student put her at an immediate disadvantage in a university system that has favored white, middle-to-upper-class students with inherent social capital from college-educated family members. Yet, her background also provided her perspective on how institutional conditions could be evolved to better include students like her, which she brought into her campus employment position to influence change. This required a tremendous amount of resilience, another element of personal factors which is explored in greater depth in another section.

Identity is influential in how individuals approach the world and how the world responds to individuals, and the undergraduate years provide ample opportunity for identity development. For developing undergraduate students, campus employment and 
the social interactions within it provided an important context for identity exploration and development. The systemic implications of intersectionality worked to privilege or oppress student employees in campus employment, whether student employees explicitly recognized it or not. Student identities influenced how student employees interacted with others and participated in workplace experiences, with both positive and negative implications for learning.

\section{Motivation}

A second personal factor which influenced engagement in learning experiences through campus employment was students' motivation. The student employees who felt they learned a great deal from their campus employment experience were motivated to grow through their position, take on additional responsibilities, and embrace challenges. Students who personally connected with the work and felt they were making a meaningful contribution were also more likely to seek out learning opportunities. Keeling (2004) asserted that learning must be considered within the larger context of who students are, what they know, their values, and how they see themselves contributing to the world. The findings from this study reflect Keeling's assessment, demonstrating that personal connection to the work is an important contributor to the learning process.

Values. One of the greatest individual motivators for on-the-job learning expressed by participants in the study was a connection between the work in which they engaged and their personal values systems. Kyle shared the effects he had previously experienced working outside his values, saying "I'm driven by my values...I find it really hard to do something if I don't believe in it. I get really sad and don't want to keep doing it. I want to change my circumstances." Kelly similarly believed that work driven by 
passion was an important motivator. "I put my best effort into work when it means something to me...I've had jobs where it's not really what I'm passionate about and it doesn't really provide any fulfillment, and it's hard to continue to show up." Working in alignment with their values kept student employees engaged in their workplace and intrinsically motivated to excel.

In her work, Shannon hoped to break down barriers to accessing the activities her program supported. She felt that the program could better be serving "women and people of color who aren't necessarily confident in what they're doing." Using this as a core motivating factor, she learned more about how best to support those groups of people and tailored her outreach efforts. "If I can get them [engaged] and have them see this is fun, that's the best feeling in the world." Her work felt meaningful to her because she worked in alignment with her personal values and saw the results of her efforts.

Kelly's position aligned with her personal values system in helping her address a perceived injustice she saw in the world on a localized level at the university. She described the sense of empowerment she felt in actively resisting:

The job was kind of a way of seeing something that I really dislike about our world and something that frustrates me, and something that used to make me feel helpless and powerless, and it provides a way to almost deal with those feelings and show that I'm not powerless and helpless.

The motivation to make a cultural change in an issue she was passionate about was a strong motivator for figuring out how to most effectively do her job and influence the largest number of people.

All of the students who felt a personal value connection to their work had some level of related previous knowledge or personal experience, which provided relevancy to 
their work and a basis from which to engage in deeper learning. Many were able to describe the ways in which they synthesized their previous knowledge with new information, gained both through the position and other life experiences, to enact change. The motivation to do the job well came from the recognition that the position provided a platform and resources to make a difference. As Angie described, "I can use my experiences and my stories and my concerns to bring it into a bigger organization that can actually do something about it." The workplace provided an avenue for taking meaningful action.

Kyle described the ways in which his background experiences and personal beliefs played into his choice to pursue employment in his department:

I took the things that I had been experiencing in my mind and in college up to that point, and felt like what I really care about is kind of the system of the world around me, and I want it to be better...So, that's why I chose the position, because I believe in it.

The personal connection he felt to his work motivated him to learn as much as possible about it, as well as ignited a passion to teach others.

Sense of Purpose. While connecting to their values allowed students to work in alignment with something that was important to them, experiencing a sense of purpose related more to feeling propelled to do something because they knew it was going to be important to the populations they served. Student employees were more invested in their jobs when they felt that the impact of their work made some kind of meaningful contribution to the bigger picture. Having a connection to the purpose of the work and recognizing their ability to influence outcomes encouraged student employees to give their best efforts. 
Sarah described how she felt the importance of providing support for parents and families of students in her position.

I think serving family members is a big role...They're the ones supporting their students, helping them either emotionally, mentally, or financially through college and through this new experience, and for some it's like the first time you've been going to college. So, I think just being in this role of being supportive, and being that like 411 kind of hub is really helpful in general, as well as inviting them to the regional events or inviting them back onto campus for these different experiences that we host.

Recognizing that her role was influential inspired Sarah to give her best at work, which in turn prompted her to seize learning opportunities.

Drew recognized the influence they held in their positions over creating a potentially life-changing experience for local youth. They pulled from personal experiences they had growing up to understand the potential impact of their work on future college students:

Going into college for me, I felt like it was really important to be able to pass skills that I learned and knowledge down and out so that people didn't feel super isolated or like they had to be inauthentic in order to be an adult in the world... There are a lot of ways where you can live authentically and still navigate the world, and I think that being able to support people in living their most authentic lives and also showing younger [people] that they're valid and that they have an existence in the world is really important. So, I developed that passion partly because I didn't see it in my own life, or really get the encouragement, until later.

Drew was energized by the prospect of providing a safe environment and helping validate the experiences of the youth they worked with in a way that was not present in their own life previously.

Multiple other participants also perceived that the work they put into their oncampus job would provide them an opportunity to make a difference in the world. Being driven by passion motivated student employees to fully engage in, learn from, and 
contribute to their work. Tyler described both what he gave and received from working with youth camps:

As a counselor and a supervisor, there's a lot of meaning behind impacting the lives of young people. It's more of a personal, emotional, mental, psychological impact that these positions have on me. But I just really like getting to see how these people grow and how they learn things from you. The impact you have on them, and the impact that they have on you as a person is pretty meaningful to me.

The tangible outcomes manifested by doing the work inspired him to hone his craft and pour more of himself into doing good work.

Shannon expressed gratitude for working in a position in which she felt like she was contributing to something bigger:

It's nice to have a job as a college student where you feel like you're actually doing something, and not just standing around pretending to look busy.

Theoretically, I could make more money and get more hours if I was working at [a grocery store], but that's just like standing there. Whereas at the end of this, I can come away and be like, look at what I did. You know, there's like a tangible outcome. And I feel like it's a lot more meaningful. And it's what I want to do long term, so it's kind of laying the groundwork for the future.

Tapping into the passion they felt for a cause provided a sense of purpose that Shannon and other student employees longed for from their student employment experience.

\section{Learning Orientation}

When asked about the skill set a person would need to be successful in their job, time and again participants identified one of the most important characteristics as a willingness to learn. Mike best described this, saying the work required an "enormous amount of investment in learning to be successful...when it comes to staying and being effective, it definitely takes a dedication to learning how things work, rather than just showing up and doing a monotonous task." Clarke (2005) contended that individuals play an active role in their learning in the workplace through interactions with others and 
meaning-making. He emphasized the importance of "learning how to learn" through taking advantage of the learning opportunities provided by the workplace, such as learning by trial and error, observation, self-study, taking on challenging projects, or rotating jobs (p. 189). Results from this study also demonstrated that students who had a learning orientation sought out greater opportunities for challenge and increased responsibility in the workplace.

By seeking out opportunities for learning and recognizing the learning potential of their jobs, student employees were able to reap the benefits of experiencing employment as a continual learning opportunity. Angie framed this orientation toward learning, saying, "You might have training, but you're never going to know everything before you start the job. So just the ability to learn, and to explore and to see what works and what doesn't work [is important]." Thus, a major contributor to experiencing student employment as a learning opportunity was student employees' inherent eagerness to learn through it.

Every student employee interviewed expressed engaging in some level of learning within their campus positions at varying degrees. However, perhaps unsurprisingly, student employees who held low expectations for learning within their position indeed expressed the fewest opportunities to learn at work. Bo, for instance, applied for his position precisely because he judged the position to carry low responsibility, preferring to fully focus his energy on academic studies. As he described, "Mostly it just sounded like a pretty like, easy, flexible job. It was like my first year of school, so I wasn't looking for anything super intensive that required a lot of my effort and time." Bo's low motivation for treating campus employment as a growth opportunity was the primary influence on 
his behavior in the workplace.

\section{Resilience}

Another characteristic the student employees interviewed attributed to workplace learning was resilience, which manifested in a willingness to actively engage in problem solving in the face of ambiguity, even when there was a chance of failure. Resilience has been defined as "the process of, capacity for, or outcome of successful adaptation despite challenging or threatening circumstances" (Masten et al., 1990, p. 426). Angie described a growing acceptance of "being okay with failing and messing up" that did not quell her intention to persist. "I think sometimes jumping into something is the best way to go." Although she set an intention, planned ahead, and attempted to anticipate what could go wrong, she remained open to the element of trial and error inherent to her work.

Tyler similarly described the experience of learning from making mistakes. "For camp, there's a lot of trial and error. I feel like you constantly mess up a lot. No one goes through ten weeks of summer camp without making a mistake and wishing they would have done something different." In both instances, the student employees learned best through participating in a continual cycle of improvement, learning from previous mistakes.

Participants exhibiting this quality described a growing trust in themselves and a belief that they could tackle challenges. Embarking upon the unknown, in some cases, caused an element of excitement and renewed enthusiasm for learning on the job. As Mike described, "Sometimes it's fun to see things break in weird and interesting ways and put everybody's heads together and think about how we're going to solve this, because some things are pretty wacky when they end up falling apart." Making mistakes was a 
daily part of his job with which he quickly grew comfortable. The ability to see a challenge as fun instead of defeating allowed him to work through problems and learn from them.

Student employees who recognized the edges of their comfort zone and pushed themselves to operate just outside the boundaries were also presented with learning opportunities. Jonathan described the feeling of moving outside his comfort zone:

There's no way to grow if you're always in the same little bubble. And once the bubble pops, now I'm in this big world of going through all these transactions that I have no idea what these numbers mean or what these words mean, and I just have to figure things out. I think it's how you grow professionally.

Amanda also portrayed herself as a person who pushed beyond the initial nerves she felt upon learning something new by tapping into her internal drive to be pushed outside her comfort zone. She shared that she would use the tactic of reminding herself that other things she had done in the past were hard at first before becoming increasingly routine, and if she could get through those, she could similarly push past her trepidation to try something new.

\section{Summary}

Sociocultural theories of workplace learning have long supported the position that the backgrounds, dispositions, and motivations of individual employees contribute to their engagement with learning (Fuller \& Unwin, 2011; Hodkinson \& Hodkinson, 2004). This research confirmed that personal factors are also important within the campus employment environment. Student employees' identities, motivation, learning orientation, and resilience influenced such things as which positions students applied for, how they interacted with others in the workplace, their eagerness to take on additional 
responsibilities, and their propensity to try things that fell outside their comfort zone. Yet, it is presumptuous to expect that personal factors are the only influence on learning through campus employment. Student employees do not passively absorb knowledge within the campus workplace, but rather make meaning through social interaction. The next section outlines the ways in which interpersonal factors contributed to learning within campus employment.

\section{Interpersonal}

Lave and Wenger (1991) asserted that workplace learning is fundamentally social and relational in nature, and can only be analyzed by understanding the collective social relations within the workplace. According to Wenger (2000), learning takes place through social interactions, with individuals participating in activities within communities and making meaning from the experience. The students in this study also expressed that formal and informal interactions at work provided a basis for many opportunities for learning. All of the student employees interviewed worked with others in varying degrees in their campus employment. Participating in a work environment in which student employees worked alongside others provided ample opportunity for ongoing learning. The interpersonal factors that participants in the study identified as facilitating learning included communication, peer coaching, observation, and interactions with their supervisors, as detailed below.

\section{Communication}

In interviewing participants one thing became abundantly clear; talking to others was an important part of the learning process. Largely informal, communication took the form of asking questions, seeking personal or professional advice, or teaching others. It 
involved horizontal communication between peers working together to make sense of their work as well as top down and bottom up communication between supervisors and subordinates. These findings are in alignment with Eraut's (2007) typology of early career learning, which outlined the ways in which work processes, such as working alongside others and problem-solving, and communications-based learning activities, including asking questions, getting information, and locating resource people, influenced informal learning through work.

Their work required that Drew maintain an understanding of the complex needs of the student populations they were serving. As part of their continued education and efforts to efficiently address the needs of that community, they would engage in dialogue and brainstorming with peers at work and with student groups outside their workplace. By talking with students who had different perspectives, they learned about the various stressors in their lives as well as "intel on the whole community." Through sharing stories and learning from the experiences of others, Drew developed empathy that enabled them to be a better advocate in their job.

Kelly also relied on dialogue to increase her emotional intelligence and ability to empathize with others. Early in her employment, she would get upset and feel disrespected if program participants would laugh during or disengage from the heavy content covered during her presentations. Through subsequent conversations with other program facilitators and her supervisor, she developed the ability to see the situation from another perspective, as she described:

For some people, their coping mechanisms when they're uncomfortable is to laugh. Maybe this person has been through an experience where now the video or the whole presentation they're experiencing triggers feelings of uncomfortability, 
and people cope by laughing because they just don't know what to do. Or sometimes people just cannot stay checked in anymore because it's a lot for them, and so they pull out their phones because they need to separate themselves. And seeing how something that caused such a quick reaction from me, immediately gives perspective where it deserves compassion and understanding, I think that's a really big example.

Dialogue provided a catalyst to learning new ways of understanding a familiar, and previously frustrating, experience.

Mike discussed how interactions with career staff across the division led him to learn skills for navigating difficult conversations. One of his positional responsibilities was to maintain the technology equipment inventory, including ensuring that users accurately reported what they were doing with their devices, which sometimes put him in the uncomfortable position of reprimanding senior staff. Despite the discomfort, he felt it was valuable to "learn how to have those conversations with people and hold people accountable for things when it is part of the responsibility of working here." The forced communication with others provided a lesson on working through challenging conversations that he will carry into future jobs.

As a non-traditional undergraduate student, Bo found himself older and with more life experience than other student employees. Through conversations, he picked up on the generational differences between him and other undergraduate student employees. He described it as "a changing culture," which he felt was more politically correct and polarized than his own background. Engaging in dialogue with his work peers gave him the opportunity to tap into his curiosities to better understand his coworkers and gain intergenerational insights.

Tyler developed a deeper understanding of himself from having conversations 
with an unlikely population, the youth campers he supervised. The interactions with campers helped him learn a lot about himself "because kids are so honest" and essentially held up a reflective mirror from which he could further ponder. He described how campers would make unfiltered comments that he initially disregarded but ultimately led to personal insights after introspection and reflection. He described a "stupid, throwaway example" of this pattern:

I made a joke, and then a kid pointed out that whenever there's a word that could have two possible meanings, you always switch up the meaning from what the person was intending to say. Which is a pretty basic observation, but I thought about it, and was like, wow yeah, I do make a ton of jokes like that. But that's something I would have never noticed about myself unless I had sat down and done a ton of introspection.

As these examples highlight, dialogue provided a powerful platform for deeper learning of complex concepts in the campus workplace and a greater understanding of self.

Engaging in interpersonal communication provided ample opportunity for social constructivism, in which student employees' knowledge and understanding was built through active participation and dialogue around shared problems or tasks (Driver et al., 1994). Every student employee interviewed asked questions as a learning technique in their place of employment. They asked questions of their supervisors, peers, other people they interacted with inside their workplace, and members of communities their work supported. Asking questions was a tactic so frequently employed that it was often the first response participants gave when asked about how they learned to do something in their workplace. Previous human resources research has suggested that novice employees should be treated as active participants in learning by reflecting questions back to them when engaging in collective problem-solving (Billett, 1996). Through providing new and 
developing student employees access to experts, whether peers or supervisors, allowed employees to express their curiosities and experience deeper learning.

Sometimes questions were used to get a quick answer to a specific problem, while other times the questions were intended to elicit dialogue to develop a deeper understanding of an issue. Amanda articulated the nuance that questions could take on multiple levels. During her first few shifts in operations, she would ask coworkers to remind her of the procedures for doing laundry and checking out lockers. Later, when she was promoted to manager, she would pepper other managers with questions to develop a more complex understanding of ways in which to approach difficult situations, recognizing that every manager handled them a little bit differently. As a personal trainer, she used questions to get to know her clients, understand their goals, address their vulnerabilities, and foster a relationship:

With a client, I have this lasting relationship with them. When I'm meeting them for the first time, that's definitely a big moment, I think, because I want our first interaction to be really positive. I want them to feel really comfortable. The first time we meet, I'm asking them things that are going to put them in a vulnerable position. I'm asking them about their diet, about their activity, what their workout routine looks like... I think it's my duty to be super compassionate and show them that through my body language and how I'm communicating with them. I'm just taking in information. There's no good or bad in my head, I'm just trying to collect these facts, and then going forward, bring that same energy to every conversation we have.

Amanda's attention to not only which questions she asked of clients, but also how she asked them, fostered mutual trust which made Amanda a better trainer to support her clients in achieving their wellbeing goals.

\section{Peer Coaching}

Informal, specific, in-the-moment advice and feedback characterized the most 
beneficial coaching received by student employees. Billett (2001) identified receiving guidance from other people in the workplace who have more expertise as an essential element of workplace learning. Many of the student employees interviewed benefitted from the guidance of senior student employees, often initiated through the onboarding process with a formalized shadow training structure and continuing through informal interactions. Not only did student employees receive coaching from both peers and supervisors, but they also in turn provided peer coaching to others. Few of the participants experienced formal mentorships, but the collaborative relationships they had with peers and supervisors performed much the same function by helping student employees in their growth trajectory through the investment of others.

Receiving feedback from peers who observed their interaction with others was a large part of the continual learning process for Drew:

If you're sitting in a room with someone and someone's coming to you with a concern or you're hearing something in a space that's problematic, do you step in? If you didn't step in, you're probably going to get people saying "why didn't you step in?". If someone's coming to you with something and you don't respond to it in the best way or you ignore certain parts of their identity in favor of others, then that can be an issue.

Though this type of feedback could make someone feel defensive, Drew accepted that it was part of the nature of their position to be made uncomfortable sometimes.

More experienced workers provided structure and supported the guided learning experience of junior employees through engaging in problem-solving together (Billett, 2001). Mike described an "organic stratification" in his workplace that ranged from inexperienced new employees to senior employees, including those who were computer information systems majors who had more developed knowledge banks from which to 
draw. He shared that he would not describe the relationship he had with senior employees as "mentor-mentee," but they did still have a level of embedded respect:

They did an enormous amount to teach me how to do my job, help walk me through complex issues that I had never seen before, and explain basic concepts, because I came into this never having done [this type of work].

The willingness of more experienced workers to share their expertise personally benefited Mike and also helped elevate the work production of the entire office.

Employee turnover is embedded into the nature of student employment, typically in an accelerated cycle of four years or less from junior to senior employee. The opportunity to teach others was another way in which student employees learned through intercommunication, as teaching others requires higher level cognitive thinking skills, requiring student employees to clearly and concisely convey procedures or concepts (Wagner \& Gansemer-Topf, 2005). During the latter half of his employment, Mike suddenly found himself a senior employee, dispensing wisdom in much the same way in which he had received it. Kyle also experienced the sensation of being suddenly considered a de facto leader, shared by nearly all student employees who stayed in their workplace for more than a term. He shared:

I got hired, and for a very brief moment, I was new... As soon as a few people graduated and left, we hired another person, and now I feel like I'm one of the most senior employees. Even if I'm not more senior than other people, I am literally older than them, and that isn't as important in the professional world, when you're out of college, but in college, it feels relevant. And so there was a brief moment of feeling mentored by my peers, but now I don't have peers who can mentor me.

Recognizing how newer employees were likely viewing him, Kyle stepped into the mentoring role to provide guidance and support for newer student employees.

In his role as lead lifeguard, Tyler also had responsibility for supervising his peers 
who were lifeguards. His interactions involved "a lot of giving feedback and doing audits. We started doing audits every single shift, so I'm always talking to them about what I'm seeing from them and asking do they feel like that's what they're doing, and debriefing." Through this dialogue, he also solidified his own understanding of the work and improved his ability to work well with others.

Peers were also helpful reflective mirrors for student employees, providing developmental feedback. Jonathan described coaching he received from a trusted peer that influenced his behavior at work. "He's told me on multiple occasions, the way you say things does not always come across the way you mean for them to." Although he admitted that it was a little upsetting to receive that piece of advice at first, upon reflection he recognized that he did have a tendency to come off as passive aggressive and wear his emotions on his face. His peer would remind him to think before he spoke before key meetings, eventually leading to lasting change. "I think that's completely changed the way that I feel like I'm acting or the way that I look at myself saying certain things, because I think that was something I definitely struggled with before." The foundation of trust that typified their relationship allowed space for growth through candid and direct coaching.

\section{Observation}

Several student employees identified that they learned through observing others within the workplace. Employees engage in observation to develop their understanding of what has to be done, how it is done, and what it looks like when it is done properly (Billett, 2014). Clarke (2005) previously identified reflective observation as a key interpersonal aspect of the workplace that facilitates workplace learning. Observation had 
both formal and informal dimensions, with formal observation orchestrated by the workplace through shadow training early in the students' employment. Informal observation occurred throughout the duration of their employment, including a process of watching and mimicking respected peers or supervisors and eventually reflecting and integrating their learning into their own work style. Several student employees were also able to bring in observations from outside the workplace to improve the service they delivered or support they provided.

Participants reflected that they relied on observation of senior employees to improve their own practice at work. Amanda made a practice of observing fellow managers, paying special attention to "How do they carry themselves? What do they say? How do they say it in those moments?" She specified that much of her learning came from "time, observation, [and] practice." As Amanda demonstrated, observation went much deeper than simple, mindless mimicry. Rather, her process better aligned with Billet's (2014) conception of observation as a higher-order cognitive activity, involving imitation, reflection, and action.

The reflective aspect of observation was an important part of the learning and behavior change for student employees. Jackie described her process for observing and internalizing lessons, "On a professional level, I like watching how they might do things, and then evaluating whether or not I would do the same thing." Tyler believed that what separated the best camp counselors from the rest was their ability to recognize "when someone else is doing something better than you and adapting your style to incorporate elements of what they're doing." Peer observation provided a mechanism for continued refinement of workplace skills. 
Heather increased her emotional intelligence in the workplace through observation of a challenging work situation which played out in real time. Early in her tenure, half of the career staff member positions were cut in a department transition, and Heather saw the impact of that change and the ensuing stress and tension first hand. The transition affected her entire employment experience, but provided valuable lessons for the future from the things she observed:

That was something that you had to be conscious of, the dynamic of knowing when to console and when to ask and when to just not touch a subject in trying to be able to read the office chemistry in that way. And then not letting it interfere with your job and how you perceive your office.

Being there while her office went through that trying period and observing the ways in which different individuals handled the situation gave her a deeper understanding of how to regulate emotions in work that she will carry into her interactions in future positions.

A few participants changed their own behaviors in the workplace based on observations made outside of work. Drew described the burnout that is common among peers who do social justice advocacy work and the ensuing lessons on self-care they learned through observing others. "Watching other student leaders...get exhausted and burnt out and learning where are we actually finding our fulfillment from... I've learned from others who have experienced worse side effects to social justice than I have how to not burn out." Drew was able to incorporate self-care into their own practice to avoid perpetuating the burnout cycle.

\section{Interactions with Supervisors}

Across the board, participants spoke warmly of their relationships with their supervisors, though each relationship had a unique characterization. The supervisor 
relationship was an important influence in the facilitation of learning for student employees in this study, consistent with other research on supervision. Ellinger (2005) found that "learning-committed leadership and management" was paramount in influencing employee learning in the workplace through creating informal learning opportunities, developing employees, supporting and prioritizing learning and knowledge-sharing, encouraging risk taking, providing positive feedback and recognition, and serving as role models (p. 400). The influence of the supervisor in facilitating learning was strong, as supervisors attended to the developmental, emotional, and academic needs of student employees. Supervisors provided mentorship, advocacy, encouragement and affirmation, and they often held unwavering belief in student employees and their ability to be successful in their jobs, even when the student employees themselves battled imposter syndrome.

Managerial Coaching. Student employees were encouraged to try new things by supervisors who were attentive to their needs and supportive of development. Managerial coaching, defined as a conversational process in which supervisors guide employees through inquiry and empowerment to figure out answers on their own, has been encouraged in the organizational management literature as an effective way in which to help employees develop (Coe et al., 2008). The results from this study confirmed that supervisors played an important role in providing encouragement and advocacy both within work and in certain instances mentored students on how to effectively handle situations outside of work.

Ellinger (2005) suggested that leaders who create informal learning opportunities for employees positively influence learning in the workplace, and data from this study 
suggests that those types of interactions also extended to the campus workplace. Shannon expected that she would have a boss who handed down a series of tasks, but was pleasantly surprised to find that her relationship with her supervisor was "much more collaborative." On a weekly basis, her supervisor checked in on her goal progression and planning, offering advice and encouragement in equal measure. This led Shannon to "feel as though they actually are interested in and care about what I'm doing. And they want to help me do what I do." This support demonstrated an investment that the supervisor had in the student employee as a person as well as in the work that was important to them.

Supervisor who were effective coaches took time to get to know student employees and support them in reaching goals. Angie and her supervisor shared a "strong supervisor/student employee bond, that gave [student employees] the confidence to make our own decisions, but also know that we have support." Her supervisor made an effort to understand her goals and encourage her in her personal and professional growth. Outside of the workplace, her supervisor also provided advising and advocacy that she believed helped her succeed as a student. "He knows everybody, so he can refer me to people, and I meet different people through him that will assist me in other ways in college later." The influence of her supervisor extended beyond the workplace into other facets of college life. Jonathan discussed with his supervisor his tendency to easily get bored when faced with the same mundane tasks and his growing discontentment with the job. His supervisor validated his feelings and together they worked on a plan to direct Jonathan toward novel challenges and work that he found more interesting.

Other student employees' supervisors were characterized as trusted advisors who helped them navigate both work and academia. Heather's supervisor was an alumna of 
the university, which she felt provided her supervisor with "a better understanding of the resources and the things that I'm going through." She felt she could go to her to get guidance on navigating university systems. Kelly also experienced support from her supervisors that extended beyond her job.

Working in the Dean of Students Office, [my supervisors] have a wide reach across this campus. They know a lot of faculty members, they know a lot of professors, and people all over the place. And because they're so invested in my life, they want to help me whenever there's something going wrong. They've talked to teachers for me, who are kind of annoying me [saying], 'Hey, that's our student director, she does a lot. Please make it easier for her, or just be understanding, hear her out.' That has helped a lot, and I think just the consistent checking in about how I'm doing in general, but also academically, and genuinely caring, makes me care about it even more.

The support her supervisors provided led Kelly to care about and respect them with such fervor that it motivated her to excel in her position.

Developers. Supervisors held an important role of developers, providing student employees feedback and ongoing guidance (Ellinger, 2005). Timely, relevant, and specific feedback was regarded as the most beneficial type, and student employees were more open to receive feedback from supervisors when they had cultivated trust between each other. As developers, supervisor guidance extended beyond feedback into encouraging student employees to take up the opportunities for learning provided within their workplace.

Jackie's supervisor was new in her position, and she often engaged student employees to understand the nuance of the job. Jackie described the ways in which feedback loops in her workplace made it a more comfortable environment in which to learn:

Sometimes our supervisor will come up, especially because I have worked here 
longer than she has, to ask us questions. We have kind of like a loop of feedback, where she'll give us feedback, and we'll give her feedback about how things she's doing or how certain things are working or not working. Since we're on the front lines, we know where certain groups are getting confused and where they're not. So, it's helped me be more confident and comfortable with talking to supervisors and people in a higher position than me, just because we're all on the same level on some things. Because sometimes, we're all very confused, so, it's okay.

Having her supervisor also show some vulnerability in asking questions when she did not know the answers provided a cue to Jackie that she was in an environment where learning was acceptable and even expected.

Tyler attributed many of the skills and lessons that he learned in his campus recreation positions to "having supervisors who are willing to give feedback." He further explained that they provide feedback that "is useful and stuff that you can actually take and build upon. It's not just a generic 'you did a good job.' They highlight what you did well... and what things you could work on and improve on.” As a CPR/first aid instructor, he received feedback from both the co-instructor he team taught courses with and also his supervisors, who would come in to observe parts of the class. He described the process of taking a piece of feedback and implementing it into practice:

They'll give me little feedback, like this group's kind of young, so that style might not work for them. You might need to do more interactive type of stuff. That's a specific piece of advice that [my supervisor] gave me this last summer after my first class, and I implemented in my second class, and it worked really well.

Tyler appreciated that his supervisors cared enough to provide specific and timely feedback, which allowed him to immediately and continually improve his practice.

Heather would go to her supervisor for advice on navigating challenging situations, both within and outside of work. "I feel like I could talk to her about anything, especially when it comes to growing my social skills and my collaborative skills." She 
relayed an instance where she was fretting about a faux pas she committed during a group project which she talked through with her supervisor. In responding, her supervisor showed compassion and empathy, asked reflective questions, and dispensed nuggets of advice to help put the problem into perspective. Heather described the effect of being able to use her supervisor as a sounding board, saying it "makes the job worth it so much more because it's not just about a job. It's about mentorship and shaping into a real professional.” In this way, her supervisor influenced both her personal and professional growth.

Kelly also expressed a sentiment shared by several other participants that her supervisors believed in her capability to effectively do the job and take on additional responsibilities well before she did. Although she applied for her position, Kelly doubted her competence and ability to successfully take on the student director role. As she described it, "My supervisors have so much faith in me more faith than I have in myself." The confidence her supervisors had in her helped her overcome the imposter syndrome she was experiencing. "Just the fact that my bosses have faith that I can manage myself and get things done in turn makes me confident in doing it, and then I do it better." Her supervisors essentially held the space of confidence for Kelly until she was ready to step into it. Supervisors who advocated and encouraged their student employees helped them develop confidence to pursue new challenges.

Affirmation and Appreciation. For student employees who had a wavering sense of self-confidence, supervisors were able to play an important role in affirming good work and showing appreciation. Ellinger (2005) has stressed the importance of providing positive affirmation and recognition to employees to support their learning 
endeavors, and it seemed especially important to student employees having early career experiences. Throughout his employment, Jonathan learned to trust his knowledge, abilities, and judgement, yet still relied on support from supervisors to remind him of his capabilities from time to time. He relayed a story of doubting a transaction he was responsible for and taking the problem to a supervisor who affirmed his judgement was corrected and reminded him, "You just need to trust yourself because... clearly you have the knowledge. You just need to believe that you have it. And then you don't have to constantly be curious if you're doing good enough, because you are." Receiving that affirmation from a supervisor was instrumental in helping Jonathan feel confident in his ability to navigate the position.

Receiving recognition for their work served to boost student employees' confidence and motivation to excel. Kelly's supervisors freely showed appreciation and offered praise when they noticed she was excelling in her position, which helped her develop a sense of confidence both within and outside of work:

The praise I get for when I do exceed expectations or standards, has really helped me build my confidence. It started with confidence in my job, and then it expanded to confidence in like my time management and confidence in my ability to juggle multiple things, and then it grew into confidence in myself.

The appreciation that her supervisors showed helped Kelly recognize her value at work and feel as though she could excel in other things as well. Angie felt motivated to do even better work when her supervisor expressed appreciation. When he expressed that he was "really proud of everyone and everything they're doing," Angie felt inspired to look for opportunities to do better and contribute even more.

As Kyle described, it plainly just felt good to receive recognition from his 
supervisor. During meetings sometimes a supervisor would "say a really heartwarming thing about how much she appreciates everybody and all the work that they've put in." When his supervisor spent time recognizing specific achievements, he noted that it would feel "really good to be on the receiving end of that and to be recognized by a supervisor in that way." Receiving that type of positive reinforcement motivated him to continue to churn out good work.

\section{Summary}

Through interpersonal interactions with others within and outside their workplaces, student employees engaged curiosities, expanded understanding, and deepened their knowledge. In communicating with other employees, they had immediate needs met as well as gained insights which challenged embedded ways of thinking. In observing others, student employees had a chance to watch how other employees did things, reflect on and internalize lessons, and change their resultant behaviors. Peers played an important role in offering corrections while working alongside each other, while supervisors managed, developed, and affirmed the growth of student employees. In these ways, interpersonal interactions motivated changes in employee behaviors, thoughts, and ways of being. The previous two sections have considered the personal and interpersonal factors which influence learning through campus employment, and the next section turns to the supportive conditions of the workplace environment.

\section{Environmental}

Workplace learning literature has suggested that organizational conditions influence the amount and quality of learning experienced in that environment (Burnside et al., 2019). The third and final component that influenced learning in the campus 
workplace was related to the environmental factors, which, in the case of campus employment was defined at the department level. Organizational elements transformed a campus workplace from simply a place where students earn money to a co-curricular educational environment in which personal and professional development of student employees is prioritized. Student engagement, and by extension student success, is influenced by institutional conditions (Kinzie \& Hurtado, 2017), and campus workplaces are no exception. Though students have a role to play in dedicating time and effort toward participating in educationally purposeful activities, the institution also has responsibility to provide outside-of-class experiences that are accessible. One of the primary purposes of higher education is to prepare students with the knowledge, skills, and personal qualities to enter a global society (Kuh, 2008), and the campus workplace can support co-curricular student learning through engaging student employees in educationally purposeful activities.

Clarke (2005) described how individual and group learning was influenced in the workplace through the organizational culture, policies, practices, and systems that make up the work environment. Collectively, these factors influence the level to which the workplace constitutes a supportive learning climate. The next section will outline the ways in which campus employment experiences were structured to create a supportive learning climate and deliver effective educational practices, contributing to the development of students' knowledge, skills, or capacity. The organizational elements are broken up into three themes: training methods, access to increasing responsibility, and the development of a learning environment.

\section{Training Methods}


Burnside et al. (2019) argued that work conditions, policies, and processes of a campus workplace shape the level of impact the experience has on student employee development. Watkins and Marsick (1993) further contend a learning environment is not created solely by designing learning activities, but rather through work structures, environments, technology, and policies. Research participants referred to both training processes and materials when describing the ways in which their workplace supported their professional development. Social constructivist training processes, in which student employees engaged in conversations with others about shared problems or tasks, encouraged them to become active participants in their own learning. Another way in which the organization facilitated learning among student employees was ensuring they had access to the necessary resources to be able to effectively perform job duties with minimal assistance. These tools for success were tangible, structured elements specifically designed to induce learning. Resources provided by the workplace included investment in the student employees through providing training, procedural manuals, historical records, and organizational tools.

Literature related to training and workplace learning has highlighted the importance of supportive training and development policies and procedures to facilitate learning which addresses both individual and organizational needs (Wexley and Latham, 2000). Data from this study support the position that effectively structured onboarding and training experiences influence student employee learning in the campus workplace.

Onboarding and Initial Training. For most participants, their initial learning experience in campus employment came through their employer's investment in onboarding and training. Onboarding plays an important role in helping student 
employees acclimate to the work environment, including a socialization process that cues new employees to the norms, procedures, and organizational culture (Bauer \& Erdogan, 2011). This took a variety of forms, from receiving a position overview at the most basic level to an intensive quarter-long experience which included classroom instruction, hands-on learning, training alongside more experienced workers, and finally culminating simulations to test retention. The quality of training varied considerably, but participants who received higher quality training felt a sense of elevated importance that influenced their motivation to excel.

Drew described the student employees in their department as "doing wildly different things." The onboarding and initial training for their position entailed "three days of eight hours of training where we talked about what is [the department], what do we do, and some basic information on how to run an event." Onboarding also covered confidentiality, professional email communications, and a brief introduction to the people employees might work with in their positions.

Jonathan and Jackie had the longest onboarding and training period of all the students interviewed. As Jonathan described, "I think the first seven weeks of the term we spent, I want to say three hours in training at the end of the week." Jackie compared the training she received in her position with peers who held other campus jobs, saying:

For my job specifically, financial controller, we spend a whole term in training. I know other student jobs on campus, you might be in training for like a week or two weeks or something like that. My friend just kind of got thrown into [her job], because there was nobody to actually train her. Whereas this one, we only hire once a year, we purposefully spend a whole term training people, you have to, at the end, take a test. It's kind of like low pressure, but it's a test. And so before you're allowed to actually do stuff on your own and not have to have another financial controller look over your stuff, you have to pass that test. We require a six term minimum commitment, because we spend so much time and training and 
learning all these rules, and all that kind of stuff. So, it's kind of intense.

The training they received consisted of both classroom training conducted by a senior controller, as well as guided hands on training. Jackie described the hands on period, saying, "One of the financial controllers is assigned to one or two trainees, and they'll go through exercises, or if customers come up, they give them to the trainees and then are there to go over and answer questions." Jonathan provided an example, "If a contract came through, and we were learning about the contracting process, whoever was working would make sure that we're at least watching what's happening, if not actually going through and doing all that." Both Jonathan and Jackie described their financial controller positions as high responsibility and the training they received not only prepared them with the skill set needed to do the job, but also set the tone for the elevated expectations the position would require of them.

Shadow Training. In describing an expansive work environment, Fuller \& Unwin (2011) highlighted the importance of treating new employees as learners and providing facilitated support toward becoming full members of the community. Further, they argue that workplace learning is embedded in everyday activities and social relations. Shadow training, in which an experienced student employee provided training and guidance to new employees within the work context, supported hands-on learning experiences. It provided student employees an opportunity to observe, work alongside, and engage in dialogue with someone who already knew the ins and outs of the job within context. Many felt that this type of training better prepared them for the nuances of the position.

Bo described his shadow-based training period was set up to "piggyback you on 
with someone else who's been working there for a while, and then you'll just kind of shadow each of the different shifts, because there's some different things that happen in the morning and the evening." In Mike's IT position, he regularly handled unpredictable situations, so part of his training period consisted of "shadowing people on appointments, because a lot of what we deal with are problems that we have never seen previously." Although shadow training could not prepare student employees for every problem they might be required to solve, it did provide a solid base and a general awareness of the resources that could later aid them in problem-solving.

Shadow training also provided an opportunity for student employees to observe how senior employees navigated the challenges that arose at work and reflect on how they might incorporate the things they learned into their own work practice. Amanda described shadowing a shift in the weight room. Noting how the senior employees she trained with handled a variety of situations, she learned "this is a good system to do this, not that I necessarily have a script, but this is how I like to approach a patron when I'm enforcing policy," which she later incorporated into her own work style. Tyler similarly implemented behaviors and attitudes he had picked up from his shadowing period to inform his own practice as a CPR/first aid instructor. "I shadowed classes and just watched...you kind of know what you like and what you don't like and that helps inform decisions that you're making as an instructor." Shadow training with other employees also provided a chance for employees to observe and listen to others and engage in discussions (Eraut, 2007). By partnering new student employees with trusted veterans, trainees were able to envision themselves in their new role and adopt behaviors that were reflective of the culture the organization hoped to cultivate. 
Department-endorsed shadow training opportunities also encouraged student employees to solidify their learning through guided hands-on experience. Sarah felt prepared to independently perform her job duties at the end of her training period due to the combination of receiving one-on-one guidance while performing many of the job tasks herself.

When I was first hired, there was actually a girl who did my same position, and I got to shadow her a lot through the process of what she was doing. I was shadowing, but also kind of like an assistant. So, I wasn't just like watching and taking notes. I was physically like doing stuff for her as well, such as compiling emails, doing stuff in Constant Contact, sending things out, working with the handbook in InDesign, and stuff like that. So there were a lot of moving parts, and I feel like it was nice being hands on like that, because I got to do it, kind of half myself.

She elaborated that ongoing shadowing occurred over approximately the first six months of her employment, long after the initial onboarding and training period.

Ongoing Training. Ongoing training, that was either required or optional, also provided a learning opportunity for some student employees. The ongoing training that student employees participated in was formal, structured, and expert-led, and intended to help student employees gain knowledge in specific areas to use within their job or transfer into future opportunities (Manuti et al., 2015). Progressively building skills over time and putting them into practice is a learning activity supported through the workplace environment (Ashton, 2004), and several participants had access to ongoing and increasingly deepening development opportunities throughout the course of their employment.

Ongoing training opportunities were sometimes used to teach students a new program or skill that they needed to know to successfully complete their job. As the 
systems within her department shifted, Heather was provided additional training related to job-specific tasks. For instance, she "received one on one training with a central marketing person" to learn how to update the university calendar with career center events. She also worked with a corporate trainer to learn about the new career services software system, who directed her to their online forum for any future questions. Mike similarly was encouraged to keep up on technology changes in order to effectively do his job well. His department sent him to on campus seminars on "how to use the new ticketing system" that was implemented department wide soon thereafter.

Other types of ongoing training were intended to build on the skills students already possessed to help student employees build competence and confidence in their ability to do the job well. The ongoing training and audits she received in her position made Amanda more confident in her ability to handle an emergency situation if one were to arise. As she shared:

I've been audited for CPR countless times, so at this point, I really do feel confident. If someone were to pass out in front of me, here's what I would do step by step...I imagine myself now, if someone were having a heart attack, I probably still would have this moment of thinking I don't know what to do. But I think eventually, after a few deep breaths, that training would kick in a bit...and in a way that comforts me a lot, because I know how to respond to this situation, and I know that if it doesn't work, I've done everything I could.

In her personal training role, Amanda was also provided opportunities for ongoing development throughout the year in continuing education courses led by other trainers. The sessions covered "various fitness topics or mental health or nutrition," which she then incorporated to enhance the experience she provided when training clients.

A few campus positions provided ongoing training opportunities for student employees to hone skills that would both help them in their current job and prepare them 
for another job either within or beyond their current department. Tyler took an opportunity provided by his department to get an additional certification, which allowed him to expand his duties within the department. "Last spring break, I took the instructor development course to become a lifeguard instructor. So now...I'm also a lifeguard instructor and CPR/first aid instructor for aquatics as well." The same development opportunity also sharpened his lead lifeguarding skills, as he described, "There's an entire subsection of that course devoted to on deck supervision and what you should be looking for, which I think kind of helped me step up my skills in that area."

Jonathan felt supported to pursue additional learning opportunities through his workplace and sensed that continual development was a departmental value. Through conversations with his supervisor, he was able to establish learning goals and put a plan in place to achieve them. Occasionally, he would receive email announcements from his supervisor's supervisor alerting him to professional development opportunities across campus that the department was willing to pay for him to attend, which led him to conclude "it's clearly important that people are learning" within his department. He also cited additional opportunities to engage in training with colleagues across campus in the business affairs office. These higher level trainings were conducted by "trainers that trained professional staff when they get hired.” These additional training opportunities not only provided him additional skills that could be used in his current position, but also were transferable to the future.

Two student employees, Tyler and Angie, shared that their department supported their participation in a professional conference related to their position. Tyler shared about the department's investment, saying "Those are things that I wouldn't have been 
able to afford on my own as an individual, but not only were they available as options, but my supervisors and departments were actively pushing me to pursue it.” Through attending the conferences, they not only increased their knowledge and skill set in their respective positions, but also had their eyes opened to the possibilities available to them if they decided to pursue a position within that field in the future.

\section{Increasing Responsibility}

For workplace learning to be an ongoing process throughout the duration of student employees' experience, it is essential that they are given opportunities to engage in increasingly challenging tasks that require more responsibility. In describing a learning curriculum for the workplace, Billett (1996) agreed that employees should move from novice to experts in the workplace through being guided through increasingly complex workplace activities which require higher accountability. Though Billett is primarily referring to line workers in his study, the sentiment extends to student employment, with environmental conditions dictating the extent to which students are permitted opportunities to grow through work activities. Participants in this study described numerous ways in which their workplaces provided structured support for their movement toward increasing responsibility, including opportunities to train others, crosstrain or seek promotions, and work with greater autonomy.

From Trainee to Trainer. As participants progressed in their own employment, they were subsequently often provided opportunities by their workplace to guide newer student employees. This was one of the most common ways in which campus workplaces allowed for student employees to move from lower to higher accountability work, and thus increase their opportunity for learning (Billett, 2001). In sharing their expertise, they 
reflected on and solidified their knowledge. Bo described the process of training a small group of new hires:

It's pretty straightforward. You just bring them in, and then the day shifts are mostly about walking around the building and navigating whatever comes through. And then for the opening and closing shifts, we open and close the gates and some of the exterior doors, so just making sure they have that down. And then walking through some of the resources that we have, just because sometimes the newer people, especially the younger ones, tend to get overloaded a little bit, so things start going sideways, and all they have to do in that situation is call somebody. That's all you have to do, because it's never going to be your problem, really. It's just a matter of getting on the phone when you need to.

Throughout his interview, Bo emphasized the resources available when situations arose that were outside the scope of his position, which is reflected in how he equipped new employees.

Mike pointed out, "My job description has never changed over the three and a half years I've been working here now." However, as his experience and knowledge of the position has increased, the expectations for how he interacts with other student employees has also been elevated.

My job definitely requires me to help other people, which is good because it is instructive to me also. Like, if I'm asked a question by somebody that seems straightforward, but then I realize I don't know how to explain it to them, then that helps me be able to better explain to other people down the line and gives me a better understanding of it.

Mike's comments demonstrate the ways in which teaching others can itself provide a learning experience for student employees.

Autonomy. The majority of participants expressed having some level of autonomy in their position, with which they were able to make decisions about how to do their job, establish priorities, or conduct their business with minimal interference. When structured and adequately supported, autonomy served as a catalyst for fully engaging in 
the work and a motivator for student employees. However, being given too much autonomy without adequate support left some student employees struggling to define their role and what success looks like in it.

Generally, as the student employees interviewed mastered the foundational job skills and became more confident in their ability to do their job well, the amount of autonomy they were afforded increased. Mike described the correlation between job confidence and autonomy in his workplace:

I'd say that my level of autonomy has definitely increased exponentially with the amount of time that I've been there. I feel comfortable enough now to receive a ticket, read what the issue is, solve it, and close it myself. Whereas, people who have been there for maybe a year, don't feel that comfortable. They'll leave stuff open to ask a lot of questions, which is ideal because that's how you learn to do your job better. So, I definitely say that autonomy increases the longer that you do it and the more comfortable you feel.

Sarah also described the autonomy present in her relationship with her supervisor, saying, "I do have the freedom, since I worked there for so long, to just check in with [my supervisor]. I don't have to be waiting around for a task to get done...I know what I'm doing." In both instances, Sarah and Mike experienced a facilitated guide path to autonomy that was supported by supervisors and coworkers and an evolving trust in their own capabilities.

Being given more autonomy in their work also influenced participants' motivation to fully engage in the work. Participants internalized the self-reliance that came along with autonomy and worked harder to ensure they succeeded. Jonathan was initially excited with the prospect of holding a new job, but once the repetition of the job kicked in the novelty wore off. "Initially, all I was doing was really basic things, trying to build those skills so I can get to the point I am now where I'm actually making decisions and 
really driving my own day." At one point, Jonathan was considering leaving his job, but after speaking with his supervisor, he was given greater autonomy to prioritize his time and work projects, which provided additional motivation to persist. By taking greater ownership of the work, he felt a greater investment in the work that made his shifts more fun and interesting.

Kelly also described her position as "a very self-motivated job," with minimal direct supervision. It was evident the depth of investment she had in nurturing the program she was responsible for planning when she described it as "almost given to me as a child, and whatever you think is good for it, do for it." Although there were expectations in terms of program outcomes, Kelly felt the power of having complete creative freedom in how she worked toward those goals. She attributed her learning about time management to this balanced combination of freedom and expectations. "Having a work schedule that doesn't feel so restricting and suffocating sometimes shows me that I have the power over my schedule, and I have the ability to manage my time well and to do everything I want to." Kelly felt the full weight of responsibility for the success of her program, which motivated her to "show up, get things done, and do them to the best of my ability." In this way, autonomy provided an impetus for continual learning to most effectively fulfill the responsibilities of her position.

Amanda worked in multiple positions within campus recreation, each with increasing responsibility. The knowledge, skill set, and rapport with supervisors she built along the way afforded her a level of autonomy from the start in her personal trainer position. She had developed a positive reputation within the department, so although she was new to the position her new supervisor trusted her from the outset and gave her "a 
little bit more free reign to just kind of get going and go for it." The opportunities for cross-training and promotion that were structured into her workplace environment provided an on ramp for taking on new responsibilities and gaining a greater sense of autonomy.

\section{Learning Culture}

If the goal of student affairs is to support and extend the university's mission and curriculum with outside-of-class experiences, it is helpful to consider campus workplaces within student affairs units as a learning organization. The learning organization's culture and learning environment is such that student employees expand their thinking through collaborative learning experiences (Caldwell, 2005). Student affairs practitioners are an integral part of establishing the culture of the campus workplace, as well as setting learning outcomes and designing workplace experiences to help students achieve those outcomes (Blimling, 2015). The campus workplaces represented in this study supported student employee learning through curating a culture in which learning was valued.

The elements that shape a culture can be complex to capture, as O'Reilly and Chatman (1996) emphasized in defining culture as a "system of shared values (that define what is important) and norms that define appropriate attitudes and behaviors for organizational members (how to feel and behave)" (p. 160). Through this definition, perception is clearly an important component of conceptualizing culture. The student employees in this study described the tangible and intangible elements of their workplace and aspects of the organizational culture that they felt made it an environment conducive to learning. This type of environment was typified by a sense that student employees were valued members of a workplace in which they could ask questions and make 
mistakes with low consequences.

Questions Encouraged. Group norms, the commonly held, but often not espoused, behaviors and beliefs shared by people in the workplace, constitute an element of workplace culture (Marion \& Gonzalez, 2013). Several student employees mentioned that feeling comfortable enough in their workplace to ask questions was instrumental to their ability to learn through work. This type of environment was cultivated by building trust among employees, lowering the intimidation barrier through supportive colleagues, continual reinforcement that seeking clarification is expected, and experiencing a sense of being valued.

As Jackie reported, "Our office has a really good environment...just having that ability to ask questions and for it to be okay to ask questions." She felt that the culture cultivated in her workplace was unique in encouraging dialogue between student employees. "Every job is always like, yeah, it's okay to ask questions, but it isn't necessarily the most conducive to being able to ask questions." She partially attributed the ability to freely ask questions in her workplace to working with other people around her own age. Even if they held different levels of responsibility or position titles, working alongside people who were about her age gave her a sense of security. Additionally, the laid back nature of her office environment, in which student employees were able to "talk to each other in a more casual way" influenced a supportive working environment that "made things less intimidating and more relaxed."

Jonathan emphasized that his workplace reinforced that seeking clarification was not only acceptable, but expected. During training he was "constantly being told you can ask questions" and encouraged to get a second opinion if he got a request to perform a 
transaction that seemed off in some way. The support he felt from supervisors and colleagues encouraged him to ask for guidance whenever he felt unclear. Heather similarly felt supported by her colleagues to seek out the people who had the expertise she needed. With multiple shifts in software systems, it took time for her to build her competence in navigating problems, but her colleagues were always willing to provide guidance. Heather felt that "it's really just about being comfortable enough to ask those questions, and they've definitely created a culture where I feel comfortable enough to ask." Workplaces in which seeking guidance was normalized encouraged student employees to engage in information-gathering and meaning-making behaviors.

Another element of the work environment that made student employees feel comfortable with asking questions was when the culture was described as friendly and supportive. Shannon shared, "It just feels very open and casual and really supportive. It's not some sort of competitive position. It's people that you're friendly with and can make jokes with." Amanda also portrayed the supportive work culture that had been created, attributing it to hiring people who are like minded in that pursuit. She described the current student manager group as "very inclusive," adding "whether you just got hired as a manager and you're still training or you've been a manager for two years, your opinions are valued, your questions are important. We're all still learning." As these examples demonstrate, inviting and acclimating student employees to social norms and supporting their sense of belonging contributed to their perception that the workplace culture was supportive of learning.

Making Mistakes. Another aspect of the workplace environment that facilitated learning in student employees was normalizing making mistakes and providing 
reassurance that mistakes are opportunities for learning. When it comes to learning in the workplace, there is evidence that there is ample opportunity to learn from mistakes within a supportive culture (Harteis et al., 2008). Findings from this study supported the notion that a workplace culture that encourages reflection on mistakes, rather than stifling or creating a sense of fear around making mistakes, facilitates learning. Student employees in these types of workplaces felt safe in proceeding, even in the face of uncertainty, because they trusted that the consequences for making mistakes were low.

A few participants expressed that their workplaces did not have any illusions that student employees would operate with the certainty of success. Although she did not work directly with other student employees on projects, Angie indicated that many of the lessons she learned in her position with the student sustainability center came from collective experience. "Just working as a team and all doing different projects, and failing and trying again...I feel like we're all learning together, and it's an experience.” Mike characterized his work environment as one which "requires you to be okay with failing." When new employees are hired, they are told "it's going to take about a year before you're not feeling like a fish out of water." Statements like this helped normalize making mistakes as a natural part of student employment, freeing student employees from the fear of judgement.

Several student employees felt encouraged to take risks or try something new due to the perceived low consequences of making a mistake. Jonathan took comfort in knowing that backup systems were in place to ensure that serious mistakes would be caught before they became problematic. Making mistakes was also an everyday occurance for Shannon, but she did not feel that any mistake she could make in her work 
would have far reaching consequences. Instead, she relied on her resources, including coworkers, to sort out problems and make corrections. Kyle expressed that there was "pretty low consequence" for making mistakes in most aspects of his work:

There are lots of different mistakes that I can make, and it won't matter because ultimately, we are a program that justifies its existence, not in the same way that the private sector does. So it doesn't hit anybody that hard when they make a mistake. It's kind of like, oh, well, it could be better if you didn't make that mistake.

Workplace environments such as these reinforced a belief that making mistakes was a natural part of the learning process, allowing student employees to seek out challenges with minimal fear if things went wrong.

\section{Summary}

Undoubtedly, the structure, culture, and affordances provided by the campus workplace environment played an important role in shaping the opportunities for student employees to engage in learning experiences. However, as Fuller and Unwin (2011) cautioned, over-emphasizing the environmental factors of the workplace can underplay the contribution of individual factors; conversely, an over-emphasis on individual factors can misrepresent the influence of the environmental context. Indeed, it is essential to understand all three factors, personal, interpersonal, and environmental, and how they interact in order to effectively facilitate learning opportunities within campus employment.

\section{Reciprocal Determinism}

The preceding sections introduced the personal, interpersonal, and environmental factors which influenced campus employment as a learning experience for undergraduate student employees. However, it is insufficient to consider each of these factors in 
isolation, as the data suggest that it is only through a reciprocal relationship between the three factors that learning is facilitated. Much like in other workplaces, learning through campus employment is not limited to a simple, single factor, but rather is best understood as a dynamic system comprising complex interactions between student employees, other people within their workplace, and the workplace environment (Fuller \& Unwin, 2011; Stasz, 2001). Thus, as student employees participate in the activities of the workplace alongside others, they are both shaped by and help shape the experience. While the previous sections broke personal, behavioral, and environmental factors apart, the following section of this chapter describes the complex ways in which they interact with each other to induce learning in the campus workplace.

To consider these dynamic relationships in the context of campus employment, it is helpful to once again consider Bandura's (1986) social cognitive learning theory. Bandura suggested that personal, behavioral, and environmental factors reciprocally interact with and influence each other to induce learning in individuals (Figure 1). Though all three are important, the relative influence of each part of the triad varies according to circumstance and context. Borrowing from the relationships present in Bandura's model provides a new way of thinking about the interactions of personal, interpersonal, and environmental factors in campus employment. Learning through campus employment does not result from any single factor, but rather is a result of a dynamic interaction between them, with each of the factors exerting more influence at times. In other words, student employees' engagement in learning through campus employment depends on student employees, the people they work with, and the workplace environment collectively influencing each other. 


\section{Figure 1}

Triadic Reciprocal Determinism

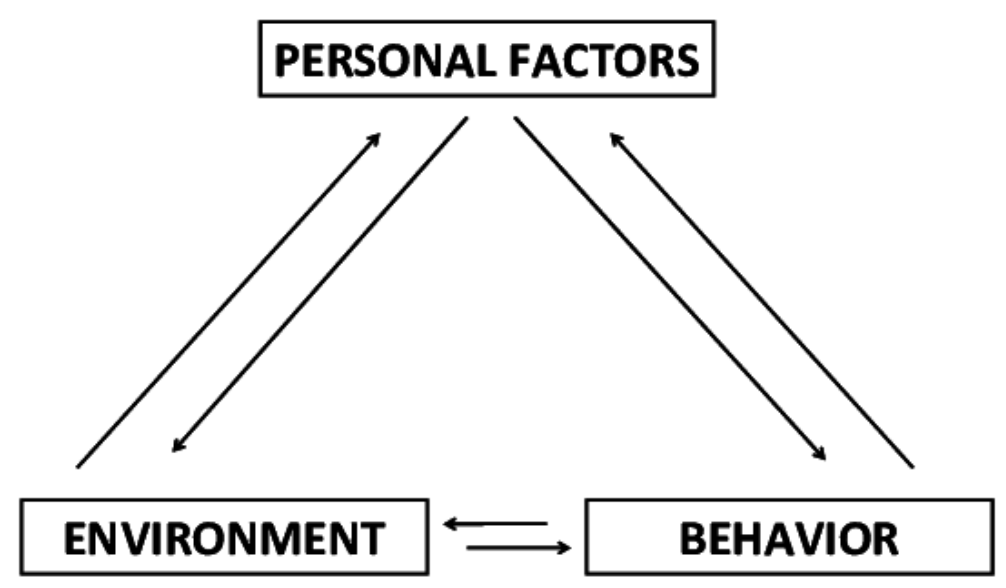

The data substantiate that personal, behavioral, and environmental factors interact with one another to influence campus employment as a learning experience, encouraging the development of academic and professional self-efficacy in student employees. Shaping the campus employment environment is important, but it is insufficient to consider the environment in isolation. The campus workplace, and the interpersonal interactions that occured within it, shaped the individuals just as much as individuals shape the workplace. Thus, for transformational learning to occur in the campus workplace, there must be mutual action between all three factors. That is not to say that one influence cannot be stronger than another at any given time. For instance, a number of personal factors may come into play over the course of a student's employment that would supersede environmental or behavioral factors, such as experiencing additional stress from a particularly challenging class. Interpersonal factors may sometimes be the predominant factor, as when a student employee observes a peer's behavior and changes 
their own to reflect what they admire. At other times, environmental factors may play a predominant role in shaping behavior, such as a looming deadline for delivering a program or being presented with a promotional opportunity. However, on the whole, it is through the confluence of personal, environmental, and personal factors that learning is facilitated.

Looking at the interactions between each of the personal, behavioral, and environmental factors sheds additional light on how they shape campus employment as a learning experience. Personal factors influence the readiness of student employees to engage in learning in the campus workplace (Fuller \& Unwin, 2011). These factors include motivations, attitude, future goals, prior experiences, and knowledge. This category also encompasses the myriad backgrounds and identities held by student employees that help shape their worldview. Self-efficacy, or students' belief in themselves and their capacity to do the work, is also influenced by workplace experiences and in turn influences the ways in which they show up to do the work.

Students' personal backgrounds, prior experiences, thoughts, motivations, and agency can be the igniting factor that influences learning activity. Kelly's previous experience with public speaking included speech competitions in high school and taking Spanish language classes. Through those previous experiences with public speaking, she started developing that skill set. When she saw her campus position advertised, her previous successes gave her the confidence to apply. Once in the role, she had many opportunities to hone that skill set through actively engaging in public speaking, debriefing experiences with other employees, and refining the ways in which she approached leading workshops. Billett (2001) posited that any learning that takes place 
within the workplace builds upon what individuals already know or have experienced, which aligned with Kelly's workplace learning experience.

Individuals are both influenced by and lend influence to behavioral and interpersonal interactions in the workplace in numerous ways. Through bringing prior knowledge into conversations, student employees elevate collective knowledge of the group. Even just by showing up as they are, individuals shape interactions with others. For instance, race, gender, and other easily identifiable characteristics factor into the ways in which student employees are perceived in the workplace, which changes the ways in which other employees interact with them (Jones \& McEwen, 2000). Students of color who participated in this research shaped the conversations that happened among colleagues, internalizing some of the lessons in a way that contributed to their own identity development.

While it is true that part of experiencing the campus workplace as a learning environment is the student employee's own participation, it also holds that the workplace environment and the people within it influence the learning behaviors of student employees. Take, for instance, the example of Amanda who was initially drawn to work with campus recreation through a personal interest in pursuing wellbeing. Once in the workplace, she learned of opportunities for cross training and promotion provided by the department. Conversations with her peers, job shadowing a fitness instructor, and observing other people in the positions she aspired to hold influenced her to apply for positions that held higher responsibility and provided growth opportunities. It is through the interactions of the positive combination of environmental, personal, and interpersonal influences that Amanda was propelled to ever deepening learning opportunities through 
campus employment.

Jonathan's experience with learning through campus employment was initiated by his supervisor during his interview process. As he described:

During my interview, my now supervisor [told me], just so you know, this is not a homework job. If you're in the office and you're clocked in, there's something for you to do. Homework and your own things are for your personal time...Initially, I wondered, do I even want this job? But I think had I done another job, I wouldn't have developed any of my personal skills or professional skills.

Throughout his employment, Jonathan experienced a number of learning and development opportunities through personal, interpersonal, and environmental interactions. He reexamined his major and future career trajectory through conversations with his peers and being given higher level accounting responsibilities. When a senior employee graduated, he asked to take on additional responsibilities in the interim. His supervisor provided him with additional challenges related to his interests when it became apparent he was no longer being challenged in the work.

If campus employment is considered through the lens of social constructivism, it follows that student employees construct knowledge through the interactions they have with others within the workplace. Not only is their own knowledge shaped by these interactions, but they also influence collective knowledge as well as potentially shape the workplace itself.

\section{Mediating Mechanisms}

The preceding description of the interactions between personal, interpersonal, and environmental factors provide a compelling case for considering campus employment as an effective student engagement opportunity. Campus employment has the potential to provide an educationally purposeful experience that develops in student employees the 
knowledge, skills, and personal qualities needed to thrive in a global society (Kuh, 2008).

Kahu and Nelson (2018) argued that student engagement results from a complex interplay between institutional and individual factors. However, they further contend that it is insufficient to consider these factors without also understanding the mediating mechanisms which impact student engagement and success. They identified four psychosocial constructs which served as mediating mechanisms in student engagement: academic self-efficacy, emotions, belonging, and well-being.

In examining campus employment as an educationally purposeful experience, it became clear that several mediating mechanisms were also at play, including work/life balance, applied learning, sense of belonging, and self-efficacy. Although the mediating mechanisms did not directly influence how or what student employees learned, they played an important part in the degree of intensity with which student employees experienced campus employment as a learning opportunity. Each of the mediating mechanisms are introduced below.

\section{Work/Life Balance}

Although several participants conceded that they might be able to make more money or gain relevant future-oriented experience at an off campus position, they expressed compelling reasons for choosing on campus jobs. Ten of the student employees interviewed made specific mention that they felt their learning was supported through their campus position because of the student-centered focus of campus employment. As opposed to off-campus employment, campus positions were structured around the academic term, with an expectation that student shifts change with regularity. In addition to having their work schedule built around academic classes, student employees also 
were encouraged in their academic pursuits through options such as flexing their schedule, requesting time off, or swapping shifts with another employee.

Work schedules set around academic class schedules on a term by term basis reinforced the student-first prioritization of campus employment. As Mike described, "Our schedules are entirely flexible. We always submit our class schedules first and then our [work] schedule is built around it, so we will never have overlapping commitments." Tyler and Kelly were given even greater flexibility to set their own work schedules and prioritize their time both within and outside of work.

Bo's work with the student union provided stable, consistent employment, but little personal challenge, which was exactly what he was looking for in his first year of college as he focused on getting into the groove as a student. He found the position "easy to schedule around and work with" and appreciated how other student employees were willing to swap shifts when needed. Another way in which his workplace supported his learning was through providing flexible hours for him to be able to regularly work at his other job, which was directly related to his art major. "They're pretty flexible on hours. I have been shooting for a couple magazines, so with scheduling shoots, or evenings or weekend trips, they've been really great to work with on hours." He credited the success of this arrangement to the relationship he had built with his supervisor, strong communication, and a mutual understanding of priorities for the future.

Heather described the deeper level of support she received for her academic pursuits when she moved from an off campus job to her position with the career center:

They're definitely flexible along keeping balance between my work and school. They understand [school is] my first priority. That was one of the biggest contributing factors to doing a work study job, because before when I was 
working [off campus], it was really hard for me to put down those boundaries with the owner.

Her primary goal was getting through college, which required her to prioritize her class schedule over any other commitments. While she had to defend her priorities in her offcampus position, she felt a natural alignment and support in her campus position.

Other participants highlighted the inherent stress of being a college student and the ways in which their supervisors prioritized their wellbeing. Drew appreciated that their supervisor recognized that student employees were humans who sometimes had other things to do. They found the attention that their supervisor gave to not overloading them when they were already stressed helpful, describing if "I have seven million things to do next week and I don't know how much I'm going to be able to get done, then she can say, I'm not going to like give you 10 tasks.” Kyle similarly was prone to elevated levels of stress, which his supervisor picked up on. Even though he had regularly scheduled hours, his supervisor offered to move his schedule around to prioritize a healthy balance and ease some of his stress.

\section{Applied Learning}

Nearly all of the student employees interviewed were able to describe opportunities to apply knowledge they acquired in academic classes at work, whether their work was directly related to what they were studying or not. As an experiential learning opportunity, the campus workplace anchored the concrete skills and abstract concepts first introduced in the classroom, and provided an opportunity for active experimentation, reflection and conceptualization (Kolb, 1984). In other words, the workplace supported student employee learning through grounding the theories or 
concepts learned from classes in practice. The campus workplace became a place where they could safely test things they learned in class with lower consequences, developing confidence they will need in their future careers.

Kelly most eloquently described the interaction between classroom learning and applied learning in her workplace through an analogy:

They're trees with different types of roots. My work is a tree whose roots are very surface level but span really far. It covers a lot of different things, but we don't necessarily get really deep into all of them. I feel like my classes are trees where the roots just go straight down. They take up a small area of space, but they go deep. And so most of it is just connecting them by taking these depths and then plugging them into this wide surface level thing, and then you start getting deeper components everywhere.

While working in her campus position, she was keenly aware of the applicability of concepts she learned in the classroom. She felt that seeing concepts in action solidified her learning and gave her a greater conceptual understanding of her programs structure, design, and administration.

Through campus employment experiences, student employees were able to use concrete skills learned in the classroom as well as experientially test conceptual concepts. Tyler described applying concepts and models learned in his business classes as he worked to develop a proposal for a new program. "I was using knowledge directly from my managerial accounting class, so basically taking exactly what I had learned in the class and applying it." He also had the opportunity to be part of the hiring process for new student staff members, which coincided with a human resources management class he was taking. He thought it was "really cool to see from an academic standpoint what is the thought process behind different strategies for hiring and interviewing, and then apply it in real life." As he went through the hiring process, he would engage his supervisors in 
dialogue to understand the choices that were made about the hiring process and then take that information back to class to further enrich discussion with other students. This back and forth process of making meaning is an example of constructivist learning (Merriam \& Bierema 2014).

Student employees made connections between their academic studies and work, even if their major was unrelated to their positional responsibilities. This was particularly true in the grounding of abstract theoretical concepts related to multiculturalism and diversity. Multiple student employees described applying diversity, equity, or inclusion concepts learned in classes in their workplace. Exposure to new or deeper thoughts in these areas encouraged student employees to examine their workplace and the behaviors of themselves or others within it more critically.

Heather reflected on the things she had learned about diversity, inclusion, hate bias, and gender bias in class to consider the ways in which she showed up for work. She was invited to represent the university at a symposium, which gave her an opportunity to practice her developing skillset. "I'm learning as I get further into my education and life experience that it's so easy to sound stupid or biased." Her work allowed her the opportunity to unpack her biases and consider how they might show up in providing customer service or other aspects of work, and thus incorporated her learning.

As Amanda grew deeper in her understanding about bias, she felt compelled to incorporate the knowledge into her workplace. She would strike up conversations with coworkers to help educate them, especially when she heard them talking about something at work that could be problematic. She worked hard to keep an inclusive mindset at the forefront and influence positive change. Though learning through work was different than 
learning in class, she felt like her workplace was a safe space to test things out.

In their cultural psychology classes, Drew contemplated "how culture affects basically your whole life," and was challenged to understand their own identity-based privilege. When workplace communication challenges arose between students who held different identities, they examined the situation through the perspective they had gained in class and recognized the source of the conflict. As they described, "I saw where I had learned stuff and then saw it being applied," although they still struggled to determine how much they should engage in the situation recognizing their privilege.

Campus workplaces provided a living laboratory for practicing the lessons learned in the classroom. As student employees applied things they had learned in the classroom at work, they solidified their learning through engagement in a real world experience.

\section{Sense of Belonging}

Feeling a sense of belonging in the campus community is a foundational need that affects student motivations and behaviors that facilitate or hinder educational success (Strayhorn, 2012). The perception of social support, connectedness, and acceptance by other members of the campus community are indicators of belongingness. Study participants expressed a common sentiment of placing a high level of importance on feeling valued and accepted in their workplace, which are common descriptions of sense of belonging. When this basic need was met, student employees were secure enough to connect with others, engage freely in dialogue, and take reasonable risks. They also expressed elevated levels of motivation for excelling in their position when they felt like other employees cared about them and their wellbeing.

Jonathan and Mike described cultivating a sense of belonging as a central tenet of 
their workplace culture. Jonathan explained that asking questions and showing interest in other peoples' lives was "something that's built into our office." Bringing personal life into the office was so embedded into his workplace that it was stated as one of the department's core values. Jonathan described the lasting impact he expected working in this type of environment to have on his life:

Working in a place that clearly respects me as a person and wants me to show up every day, as in bring whatever version of me is the normal version into the workplace, I know that's something I want to look forward to in my career with a future employer. And without this experience, I probably wouldn't have figured that out.

In a similar vein, Mike characterized his workplace as "familial," where colleagues "genuinely care about the wellbeing of their student workers." Although the team he worked with was diverse, they managed to form a cohesive unit.

Mike also shared the response he received from coworkers after a personally difficult time in his life:

A couple months ago, I had a family member die. So, I wasn't at the office for a week and generally had a pretty tough time, and that corresponded with me getting a lot of emails from my coworkers and stuff like that checking in on me and seeing how I was doing. So, it's definitely supportive and students are very much included in all aspects of what's going on.

Sharing advice with student staff members and imparting wisdom, both related and unrelated to work, was also commonplace and appreciated in his department. Mike attributed the cultivation of the supportive work culture to the daily exercise of relying on each other, a commitment to candor, and a shared appreciation of a diversity of opinions.

Kelly characterized her workplace as an "extremely welcoming," inclusive, and "warm" environment which provided "an incredible network of support" for her and the other student employees. She felt that her workplace prioritized employees' mental, 
physical, and emotional health over everything, which led supervisors to be understanding when employees felt overwhelmed by school and other obligations or made uncharacteristic mistakes. Knowing that her workplace fully accepted her, human flaws and all, helped Kelly "feel really valued as a person, instead of like a worker bee." The impact of her supportive work culture was so salient for Kelly that it was a consistent presence interwoven throughout the duration of the interview whenever she was asked to describe to whom or what she attributed lessons she learned at work.

Drew attributed the cultivation of trust within their workplace to the shared identities of coworkers and a mutual general understanding of the inherent struggles within their identity-based community. They described a "natural support system" that evolved from recognizing that they likely have some "shared traumas" that each of them understand without necessarily needing to know all of the details of each person's individual experience. As they learned more about each others' lives, their connection deepened, making it "easier to be somewhat vulnerable with our emotions in a meeting space, or with [our supervisor] or each other."

Another aspect of belongingness that many of the student employees interviewed experienced from campus employment was the feeling of being connected to a group of people on campus with whom they naturally fit in. Through their campus employment, this sense of community helped them feel secure in their place at the university and made them want to engage more deeply. Kyle expressed this most directly, stating that what his job means to him is "belonging." He elaborated, sharing "I really like belonging to the crowd... My supervisors are really good people, they treat me well. They treat me like family. My coworkers are really good people. I feel really good there." Experiencing a 
sense of belonging allowed student employees to more fully experience student engagement in the institution, an important indicator of student success.

Kelly expressed a feeling shared by several other students that working her campus position "made a huge school a lot smaller." The positions had this effect through cultivating relationships within the workplace and encouraging integration into the campus community. The workplace environment in many instances, provided an opportunity for developing meaningful relationships with coworkers, and Jonathan and Amanda shared that they made some of their best friends through their workplace. Students who held externally facing positions also benefited from interaction with students outside the workplace. Jonathan was surprised to find that other students that he had helped in his position knew who he was when he passed them on campus. Bo also felt that connecting with coworkers and being plugged into the life of the campus community was helpful for integrating into student life after spending a few years in the military. He felt that "having that outlet to get a bit involved with the students and get to know some students a little bit more has been helpful in that transition." Interactions with student groups also provided him an overview of options to get involved.

Amanda felt like she was "home" when she was at her campus workplace. She described the process she went through to come to feel like she had found a place where she belonged. Through her first few years of college, she "never really felt very grounded with a group of people." Though she engaged in the honors college and joined a sorority, she did not feel a sense of connection with the people around her. When she started working with campus recreation, she started to feel like she had found a place where she was "part of the team," and she slowly started to grow into that community. Working 
alongside coworkers and developing shared experiences brought her into a group of people with whom she felt a "deeper connection." After spending three years and working multiple positions in the department, she described working with campus recreation as her defining experience at the university.

\section{Self-Efficacy}

The belief that individuals hold about their capabilities to perform duties and address challenges in a given situation was conceptualized as self-efficacy by Bandura (1977). Although related to confidence, self-efficacy is more specified to specific tasks or situations, such as those found in the campus workplace. It is an important concept in student employment because it impacts the ways in which student employees approach workplace challenges and their belief in their power to influence outcomes. Developing self-efficacy through the course of their employment was a component of student employees' personal agency in the workplace. Self-efficacy differed from confidence in that it was specific to the job duties and task at hand, rather than a general belief in oneself.

Several student employees described the start of their employment as a time of low self-efficacy, expressing hesitancy at performing job tasks and a lack of trust in themselves. Mike reflected on his growth in self-efficacy from the start of his employment:

I'm definitely not afraid of our job, because the first time that I set up somebody's actual workstation, I remember feeling a lot of pause with what I was doing and just general uncertainty if anything I was doing was correct. Because at first, basically nothing you do is correct entirely. I remember the first couple weeks I thought to myself over and over and over again I should find a different job because I don't know if I'm cut out for everything that we're doing here. But after those first couple weeks it started to get easier and easier as time went on. 
As Mike pointed out, student employees built self-efficacy over time as they experienced success in their positions and began to internalize the belief that they had the knowledge and skill set to successfully navigate work situations.

Student employees who described a sense of self-efficacy were willing and eager to take on novel challenges and tackle complex problems in the workplace. Jonathan described the influence of self-efficacy, saying "knowing to trust myself and trust my own knowledge and ability, that's a really powerful thing to experience.” Student employees with self-efficacy also felt better prepared to operate in the face of ambiguity. As Angie shared, "I've gotten more confident in kind of being the guinea pig. I have a lot of power to shift and shape my own ideas and other peoples' collaboratively." Additionally, employees expressed learning to trust their instincts and advocate for themselves. For instance, as she mastered job tasks and learned to trust herself, Kelly lobbied for additional job responsibilities, while Amanda and Tyler both sought out promotional opportunities to further develop their skill sets.

Shannon, Kelly, Angie, and Drew all described a strong locus of control over whether they succeeded or failed in their position. Shannon described needing a lot of "initiative, creativity, [and] follow through" to navigate her newly created position. Drew described feeling a burden of responsibility for delivering a successful major event, which they felt they could directly influence through their efforts.

Going into it, I was confident that I had a lot of the skills to do it, but then I did so much on my own that [I realized] I can do a lot. I have a lot of good skills and I should be marketing myself even better.

Although Drew expected that the program would be a reflection of their efforts going into 
it, their self-efficacy grew when they realized just how much of an impact their talent and skills lent to the quality of the event.

It might seem that as self-efficacy grows the impetus for further learning would diminish, but the opposite was true for participants. As students grew in self-efficacy, they developed a greater awareness of the limits of their knowledge and skill set, but felt better prepared to engage in problem solving and seek out resources. Amanda would remind herself "you probably know more than what you think; you're actually probably more prepared than you're giving yourself credit for," which gave her the confidence to approach new situations believing that she could successfully navigate them while also using her resources to address challenges.

Along with self-efficacy came a willingness to ask questions and seek guidance from other people with greater expertise or experience. Angie described liking to have "a rough plan...of what I'm doing, and seek advice and make changes as needed along the way while I get to wherever I'm going." Kelly similarly described trusting her intuition to guide her decision on whether to handle situations on her own or ask her supervisor for help. "A lot of my job is noticing little things, and knowing when something's big enough to go to my supervisors and ask them about it... or [when] I can just take care of or make a decision on something." Both students recognized the importance of asking for help on occasion to successfully navigate their jobs.

\section{Chapter Summary}

The purpose of this study was to understand the individual, social, and organizational factors that influence campus employment as a learning experience for undergraduate student employees. While previous research on campus employment has 
provided inconclusive results on its effects on student success (McCormick et al., 2010), the findings from this research clearly indicate that campus employment experiences have the potential to positively influence student learning outcomes. Through participation in a variety of workplace activities, student employees indicated development in numerous competencies in alignment with liberal education goals and future career readiness, including communication, professionalism, critical thinking, problem solving, leadership, teamwork, and collaboration.

The study was conceptualized in accordance with sociocultural theories of workplace learning, and the data substantiated that individual, social, and organizational factors were also prevalent in learning through campus employment. Personal qualities, including student employees' identities, motivation, learning orientation, and resilience, influenced the positions that students selected for employment, their propensity to take on additional responsibilities and seek novel experiences, interactions with others, and how passionately they engaged in the work. Interpersonal interactions provided coaching and direction, challenged students to see different points of view, encouraged healthy amounts of risk, and provided necessary support. Finally, the campus employment environment afforded students growth opportunities through structural elements, including scaffolded training and opportunities for increased responsibilities, and a learning-centered culture.

Further, the findings demonstrate that while the personal qualities of individual student employees, the conditions of the workplace, and the interactions that go on within it matter, independently they insufficiently explain how learning occurs through campus employment. The findings clearly showed that learning is facilitated through reciprocal 
relationships between personal, interpersonal, and environmental factors. The fluidity of the relationship between factors implied that there is no one set way to structure student employment that guarantees it will be a learning experience for student employees, but rather consideration of the dynamic interplay between all three factors must be given to fully understand and realize the learning potential within campus employment.

Finally, four mediating mechanisms were identified, which when experienced by student employees intensified campus employment as a learning experience. These factors were work/life balance, applied learning, sense of belonging, and self-efficacy. Though learning was not directly attributable to any of the mediating mechanisms directly, each played a powerful role in helping student employees feel more supported and valued by others in their workplace, invested in their work, or capable of taking on more challenging assignments.

Researchers have long assumed that employment during college necessarily competes with undergraduate students' academic success (Pascarella \& Terenzini, 2005; Pike et al., 2008). However these findings shed new light on campus employment, demonstrating that when operationalized effectively, campus employment can be effectively facilitated as an educationally purposeful activity, complementing and enhancing the formal academic curriculum through co-curricular learning. These findings are particularly crucial in a time when a higher percentage of undergraduate students are working a greater number of hours, primarily out of necessity to support their academic expenses (Cheng \& Alcántara, 2007; King, 2006; Tuttle et al., 2005). Experiencing campus employment as an educationally purposeful activity is especially important for vulnerable student populations, including students who are first generation, racial 
minorities, or from lower socioeconomic status families, who are more likely to work and less likely to participate in other types of student engagement opportunities (Choi, 2018; Tuttle et al., 2005). The next chapter situates findings in the literature, introduces the Campus Employment Reciprocal Learning Model, describes implications, and provides recommendations for effectively facilitating campus employment as a learning experience. 


\section{CHAPTER 5}

This research study explored the ways in campus employment comprised a learning experience for undergraduate student employees. The study was framed around the primary research question: What are the individual, social, and organizational factors that influence campus employment as a learning experience for undergraduate students? The qualitative study was conducted at a large, public university in the Pacific Northwest, and the research participants included 13 undergraduate student employees who worked for three terms or more in student affairs units.

The vast majority of previous research on student employment has portrayed it solely in competition with academic achievement. These assumptions have led to numerous studies, producing largely inconsistent results (Pascarella \& Terenzini, 2005; Pike et al., 2008). Further, the assumptions have been detrimental to considering the ways in which student employment experiences may make potential contributions to the student experience. This study makes an important contribution to the literature by demonstrating that campus employment can support and extend learning experiences for undergraduate student employees, and providing a model for facilitating campus employment as an engagement opportunity.

\section{Discussion}

The evidence suggests a strong case that student employment shares many of the same educationally beneficial qualities as other high impact practices. High impact practices require the application and transfer of learning across contexts and the integration of learning from different arenas (Hansen \& Hoag, 2018). Through their campus positions, student employees were encouraged to apply specific skills, theories or 
concepts learned from classes in practice. Through this experiential learning process, student employees gained additional insights and anchored conceptual frameworks to tangible outcomes, deepening their understanding.

The research also demonstrated that campus employment contributed to student employees' sense of belonging within the campus community. Developing deeper connections to supervisors, other staff members, and peers in their campus workplace made student employees feel like valued members of a large campus community. This ties into another quality of high impact practices, encouraging meaningful interaction with faculty and peers (Hansen \& Hoag, 2018), and is important because it addresses a fundamental need in students that affects their motivations, behaviors, and ultimately chances of student success.

If the primary goal of exerting time and effort in educationally purposeful activities is to help students gain competence, develop confidence, and become more fully immersed in learning (Kuh, 2008), campus employment clearly has that potential. As the data show, student employees developed self-efficacy through their campus positions as they learned their jobs and grew increasingly comfortable navigating situations in which there was not a clear path forward. By learning to trust themselves, student employees acted upon their curiosities, sought out additional challenges, and recognized the edges of their understanding to know when to ask for help.

Effective campus employment practices have the potential to enhance undergraduate student employees' overall student experience as well as transformative learning through cognitive, personal, and professional development (Keeling, 2004). Engagement in campus employment increased students' sense of belonging to the 
institution and personal self-efficacy. Campus positions provided structural support for employees to succeed as students and apply things they learned in class in a real world environment. Through their student employment experience, undergraduate students in this study perceived development in competencies related to transformational learning and career readiness. As they developed these competencies, they also learned a lot about themselves, including identity development.

McCormick et al. (2010) previously identified participation in an applied learning environment, developing a sense of community and a greater connection to the university, a deepening support network, and the development of transferable skills as benefits of student employment, the current research demonstrated that these aspects of campus employment are not simply perks. Instead, receiving support for balancing work and school, experiencing a sense of belonging, applying things learned in class in the workplace, and developing self-efficacy were powerful mediating factors which intensified student engagement in campus employment as a learning experience.

Thus, when done well, campus employment has the potential to facilitate alignment between an emerging academic and professional identity in student employees, preparing them for the next steps in their life. It is uniquely poised to support both student engagement and co-curricular learning experiences for students who come from historically underserved student populations, with implications for student retention and success beyond college.

\section{Campus Employment Reciprocal Learning Model}

Based on findings from this research, the following Campus Employment Reciprocal Learning Model is proposed to explain the conditions that facilitate learning 
experiences in campus workplaces (Figure 2). The model depicts the dynamic and complex interactions of personal, interpersonal, and environmental factors, as well as mediating mechanisms that support student employee learning and development.

\section{Figure 2}

Campus Employment Reciprocal Learning Model

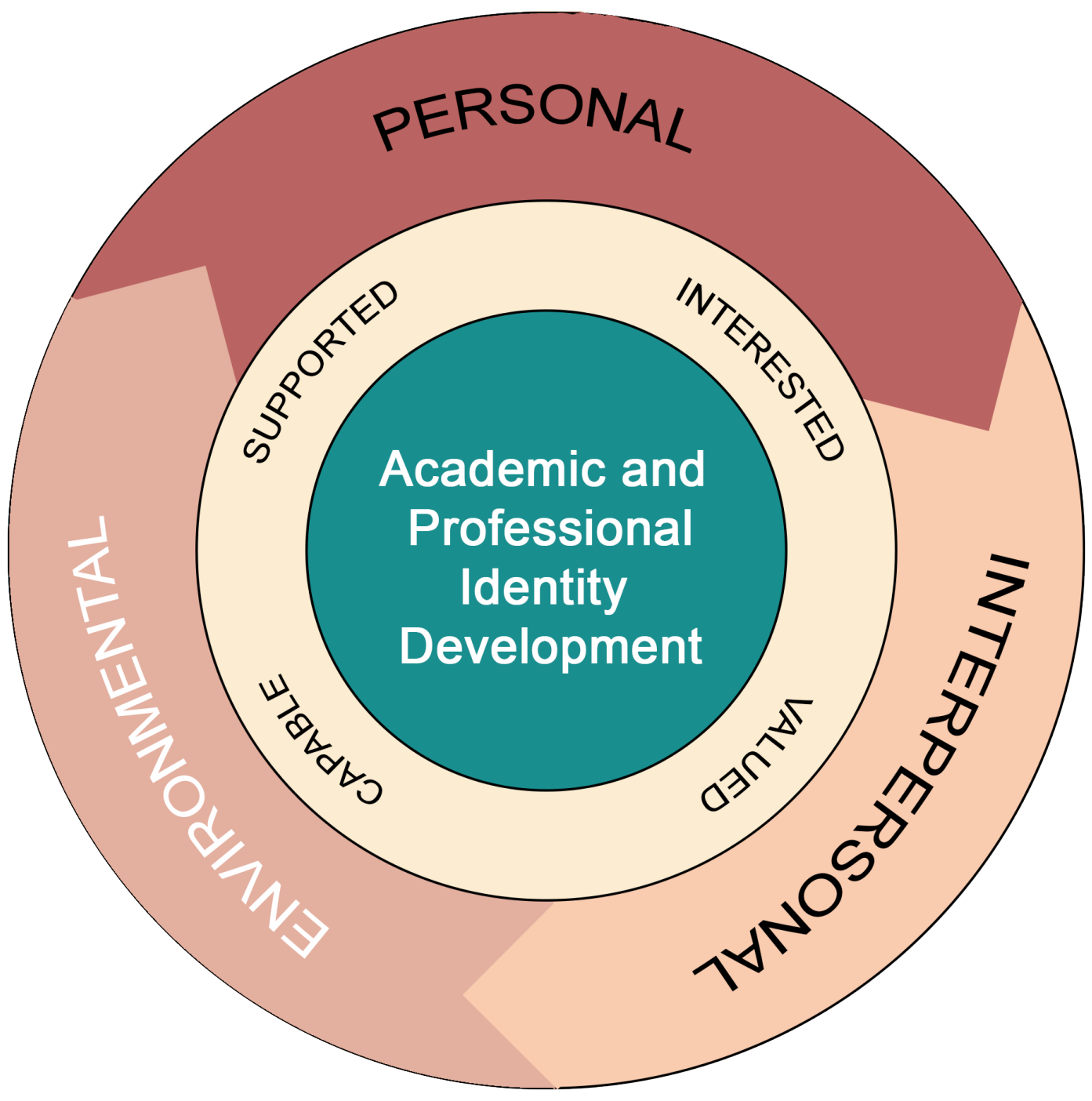

The outermost layer of the model represents the three influential factors that facilitate and support learning within the campus workplace, categorized as Personal, 
Interpersonal, and Environmental. Though each of these factors are defined independently, it is through a dynamic reciprocal interaction between them that opportunities for learning are induced. The middle layer of the model outlines the mediating mechanisms, Supported, Interested, Valued, and Capable, which increase or decrease the intensity with which student employees experience campus employment as a learning opportunity. At the center of the model, the inner circle represents a student employee's Academic and Professional Identity Development, which is continually being refined through the perimeter interactions. In the following sections, the model is further broken down and explained.

The Campus Employment Reciprocal Learning Model provides a framework for campus employers to structure efforts to encourage and enhance co-curricular learning in campus employment experiences, focusing on institutional responsibility for creating campus employment structures that purposefully facilitate personal and educational gains in undergraduate student employees, while also inviting student employees to be active participants in seizing opportunities afforded to them. This model may be used by researchers and practitioners as a launching pad for further consideration of maximizing the campus employment experience as an educationally purposeful activity.

\section{Influential Factors}

As this research has demonstrated, personal, interpersonal, and environmental factors are all important influences on how and whether campus employment is experienced as a learning activity. Drawing from social cognitive learning theory and Bandura's (1986) model of reciprocal determinism, the model suggests that learning is induced through campus employment as a result of bilateral relationships between the 
three influential factors. Each factor is outlined independently below, with the intent of describing how each influences campus employment as a learning experience. Exploring the influential factors independently leads to a better understanding of how they interact with each other to provide meaningful learning opportunities for student employees. The sociocultural perspective of workplace learning takes a systems perspective, considering individual behavior and cognition in conjunction with interactive social meaning-making that occurs within the work environment (Stasz, 2001). The degree to which any single factor is influential varies over time and context, and making changes in one can lead to changes in another, shaping the overall campus employment experience. Each of the influential factors are explained in the following sections.

\section{Personal}

Personal factors are those which are unique to student employees individually, some of which are fixed and some which can be changed. As the data showed, personal factors which influence student employees' capacity to learn through campus employment include identity, motivation, learning orientation, and resilience (Fuller \& Unwin, 2011; Jones \& McEwen, 2000; Keeling, 2004; Clarke, 2005). The prior experiences and knowledge that individuals bring influence which campus position they select, their expectations for gaining something from student employment, and the ways in which they approach their work. Personal characteristics and identities also shape the employment experience through implicitly or explicitly affecting interactions with others (Jones, 2009).

\section{Interpersonal}

Interpersonal interactions serve as the catalyst for shared meaning-making and 
social learning in the campus employment environment (Wenger, 2000). Communicating with others, both laterally and horizontally, provides opportunities for student employees to engage their curiosities, seek clarification, share expertise, and receive feedback and coaching. Social interactions not only serve the role of information-gathering, but through interactions, students also come to learn more about themselves (Jones \& McEwen, 2000). Interpersonal interactions can also happen through observing others within a social environment to see how they behave, interact with others, and react to novel situations, among other actions (Billett, 2014). It is through interactions and working alongside others that collective meaning-making takes place.

\section{Environmental}

The structural elements, conditions, and culture of the campus workplace influence the amount and quality of learning that student employees experience (Burnside et al., 2019). Student affairs units have long held responsibility for facilitating cocurricular learning through effective design of outside-of-class experiences (Keeling, 2004). Certain environmental components within the campus workplace stand out above others in promoting a supportive learning climate and effective co-curricular experience. These include training methods, access to increasing responsibility, and a supportive learning culture.

\section{Mediating Mechanisms}

The second circle in the diagram represents the mediating mechanisms, which when experienced by student employees increase the intensity of their engagement in learning through campus employment. Student employees are more likely to be engaged in learning through campus employment when they feel supported in their academic 
pursuits, interested in extending their learning through employment, valued by other people in the workplace, and capable of completing challenging work assignments. Each of these mediating mechanisms is explained below.

\section{Supported}

Student employees feel supported when their campus employers recognize the importance of their academic studies and provide adequate flexibility for employees to succeed as students. Student employment, like any other campus activity, should make a valuable contribution to student employees' educational experience (Suskie, 2015). Campus employment can support student engagement in learning by taking a studentcentered approach. Through alignment with the larger mission of the institution, campus positions can support students in their attainment of educational goals (Keeling, 2004). Recognizing that students who work have higher levels of anxiety than their peers (Mounsey et al., 2013), campus workplaces can be structured to offer flexibility to accommodate academic obligations. Supervisors can also play an important role in helping connect student employees with the numerous support services available at the institution.

\section{Interested}

Connecting and extending learning beyond the classroom is a primary goal of student engagement, and students in this study were able to identify many ways in which they applied learning from their academic courses in their work.. Campus employment provides an experiential learning opportunity in which students can apply classroom learning and explore curiosities when the stakes are relatively low (Kolb, 1984). Student employees should be supported in connecting their student employment experiences to 
academic curricular experiences and future goals (McClellan et al., 2018). According to Kuh (2008), effective co-curricular programming requires students to reflect on their experiences within and outside the classroom, integrate what they have learned, and apply it toward the fulfilment of future goals. Within the campus workplace, practitioners should consider ways in which student employees can integrate classroom learning to make the experience more meaningful.

\section{Valued}

The steady, consistent presence of the campus workplace creates a prime opportunity to cultivate a sense of belonging in student employees. Belonging has long been established as a foundational need, affecting student motivations and behaviors that facilitate or hinder educational success (Strayhorn, 2012). Student employees experience belonging when they feel valued, socially accepted, and connected to others in the campus workplace. Academic and social integration is especially critical for minority students (Fischer, 2007), and the campus workplace can be shaped to help these populations thrive through creating a culturally relevant and responsive environment.

\section{Capable}

Finally, enhancing students' sense of capability to tackle challenges in the workplace provides them with greater confidence to engage in learning experiences. Selfefficacy plays an important role in influencing learning behavior in student employees. As described by Bandura (1997), "Unless people believe they can produce desired effects by their actions, they have little incentive to act" (p. 2). Self-efficacy and engagement support one another. As student employees' self-efficacy increases, so does their likelihood of engagement. Further, as student employees engage in supported learning 
activities in the campus workplace, their self-efficacy grows. With proper structure and support, student employees transform from individuals who are heavily reliant on the encouragement and advocacy of others to push them into growth situations to individuals who believe in their own capabilities. Increased self-efficacy influences the ways in which student employees approach work and believe in their abilities to accomplish challenging things. When the workplace provides opportunities for student employees to succeed through engaging in stretch assignments with the proper support of supervisors and peers, student employees internalize the belief that they can trust in their knowledge and skills to carry them through hard things.

\section{Academic and Professional Identity Development}

The center circle of the model represents the ultimate goal of facilitating student employment as a learning experience, which is helping student employees develop their academic and professional identity. When done well, campus employment has the potential to support student employees in becoming lifelong learners. It has already been established that campus employment develops numerous competencies that align with the goals of higher education and career readiness, including interpersonal communication, professionalism, critical thinking, problem solving, leadership, teamwork, and collaboration (Strauss \& Terenzini, 2007).

The campus employment experience also has the potential to influence who the student employee is becoming and how they interact with the world, and in this way can lead to a transformative learning experience (Keeling, 2004). As student employees engage with, reflect upon, and make meaning of their campus employment experiences, they not only acquire knowledge, but also experience personal growth (Kahu \& Nelson, 
2018). Student employees should emerge from their campus employment experience with new perspectives that influence their civic, social, or career experiences into the future.

\section{Implications}

The model suggests that individual, interpersonal, and environmental factors reciprocally interact with and influence each other, ultimately impacting whether and how student employees realize campus employment as a learning experience. Thus, learning through campus employment is not attributable to any single factor, but rather is a result of the dynamic interactions between factors, with the relative influence of each part of the triad varying according to context (Bandura, 1986). Of critical importance is recognition that changes made in any one of the three factors affect the whole system, either positively or negatively. For campus employment to be effectively operationalized as a learning experience, it is imperative to recognize each factor's components and consider the ways in which they impact the entire system (Table 4).

\section{Table 4}

\section{Influential Factors and Related Components}

\begin{tabular}{|l|l|l|}
\hline Personal & Interpersonal & Environmental \\
\hline Identity & Communication & Training Methods \\
Motivation & Peer Coaching & Increasing Responsibility \\
Learning Orientation & Observation & Learning Culture \\
Resilience & Interactions with Supervisor & \\
\hline
\end{tabular}

Recognizing that learning occurs as a result of the interactions between personal, interpersonal, and environmental factors, practitioners can effectively leverage influence to optimize learning conditions within campus employment. The following sections consider ways in which components within each influential factor can be elevated and 
enhanced by interactions with others.

\section{Elevating and Enhancing Personal Factors}

Supervisors play an important role in shaping both the campus employment environment and interpersonal interactions with student employees. Ongoing, developmental training should be an expected part of effective supervision practice (Stock-Ward \& Javorek, 2003). Supervisors should become familiar with learning and developmental theoretical models, such as social constructivism and multiple dimensions of identity (Jones \& McEwen, 2000) and routinely incorporate them into their supervision practice. Additionally, supervisors should get to know individual student employees, making a special effort to understand who they are, what they value, how they are motivated, and their current and future goals. Using these insights, they can facilitate learning activities and interactions that provide adequate challenge and support.

\section{Identity}

Considering that student employees' identity is an important contributing factor to how they interact with and experience the campus workplace, supervisors must work to ensure they are facilitating an equitable and inclusive employment program. Supervisors should commit to expanding their knowledge, understanding, and ability to work with individuals who have different cultural or identity-based backgrounds (Roper, 2011). Recognizing that undergraduate students are in a unique time of identity formation (Chickering \& Reisser, 1993), the workplace should positively support identity exploration and explicitly reaffirm a commitment to diversity and multiculturalism.

Critically examining their campus workplace, practitioners should also actively work to disrupt and dismantle systemic oppression that student employees may 
experience due to intersectionality. In discussing the potential benefits of intersectional thinking on campuses, Barnett and Felten (2016) observed that it allows professionals to "be attentive to both the complexity of individual experiences on campus and the organizational structures that make it possible (or seemingly impossible) for us to engage deeply with difficult questions of diversity and inclusion" (p. xv). Considering intersectionality invites practitioners to not only become aware of the oppression of marginalized groups, but to actively work toward social justice. The campus workplace environment plays an important role in shaping the perception and experiences of students with marginalized identities. As such, student employees should not be put in a place where they are leaned on to solely educate coworkers or advocate on behalf of others who share their identities. Additionally, workplaces should work toward actively hiring a diverse and representative group of student employees and career staff while cultivating a welcoming and inclusive culture.

\section{Motivation}

Student employees enter campus employment for a number of reasons, and consequently may or may not initially be motivated to learn through the experience. Practitioners can support learning through getting to know student employees and developing an understanding of their motivations (McClellan et al., 2016). When possible, workplace flexibility should allow for student employees to pursue projects or other opportunities that connect to things they feel passionate about. Throughout the recruitment and onboarding process, practitioners should clearly articulate the purpose, goals, and expected learning outcomes of the positions for which they are hiring (Suskie, 2015). 
Student employees are more likely to engage in learning through campus employment when they connect with a sense of purpose. Campus employment environments can help facilitate the alignment between personal values and workplace mission through being explicit about the workplace's mission and vision in written materials and in-person interactions. When student employees have a deeper understanding of the core purpose of the work, they have an opportunity to assess how that fits in with their own values and worldview, as well as identify the ways in which their specific job contributes to fulfilling the mission, thus allowing students to identify purpose in their work.

Supervisors also play an important role in helping student employees realize their potential through campus employment. Through providing coaching, feedback, and encouragement, supervisors can increase students' motivation and confidence to try new things or take on challenges (Ellinger, 2005). However, It would also be wise for supervisors to assess student employees' growth needs through their positions, recognizing that a mismatch in high motivational jobs and a low growth needs may be initially distressing or dissatisfactory to the student employee (Hackman \& Oldham, 1976). Supervisors who invest energy into getting to know their student employees build trusting relationships in which even greater depths of guidance and development emerge.

\section{Learning Orientation}

Perhaps one of the longest-lasting impacts campus employment can make on a student employee's life is cultivating a love for lifelong learning (Keeling, 2004). The workplace environment can support this through being explicit about learning outcomes from the start of employment, scaffolding work tasks so they build upon previously 
learned skills, and providing reflective opportunities. Supervisors can have conversations with student employees, challenging them to set expectations for themselves on what they hope to get out of their employment experience and together thinking of ways in which they can be provided opportunities to develop the things identified.

\section{Resilience}

With any kind of learning, setbacks are bound to occur, and campus employment can help student employees develop resilience to navigate through challenging times. Supervisors who operate in alignment with Sanford's (1966) theory of challenge and support can focus on providing growth opportunities for student employees. Supervisors can ensure they are encouraging student employees to work just outside their comfort zones, which provides an adequate, not overwhelming, sense of challenge. The workplace culture should also be supportive of cultivating a growth mindset in student employees, encouraging them to continue to try when things get hard, and thus instilling the belief that abilities are malleable, rather than fixed (Hochanadel \& Finamore, 2015).

\section{Elevating and Enhancing Interpersonal Factors}

Interpersonal factors can also be elevated and enhanced to support the learning system in campus employment. Through their interactions with other people in the workplace, student employees learn from and with each other (Driver et al., 1994). Interactions can be formal or informal, and can include receiving guidance, coaching, mentorship, observing others, or working alongside others. Through interpersonal interactions, student employees also help shape the work environment. Students who have outward-facing interactions learn intel on perceptions of their workplace that they

can bring back to improve programs or services provided by the department. Interactions 
within the department help to shape the workplace culture. The ways in which people are treated when they make mistakes shapes the perception of how safe it is to fail in the workplace, either inhibiting or promoting a willingness to think and act creatively.

\section{Communication}

The campus workplace environment can stifle or promote interpersonal interactions in a variety of ways. Student employees who are encouraged to socially engage with one another through informal interactions often feel more comfortable with asking for assistance in work-related problems (Eraut, 2007). Flattening the workplace hierarchical structures can also encourage student employees to contribute to a deepening collective knowledge and honor their opinions and expertise. The environment can also facilitate opportunities for on-the-job coaching and feedback from peers or supervisors through structural elements, such as shadow training or regularly scheduled evaluations. Even the physical space within a workplace can influence the opportunity for student employees to learn from observing others.

\section{Peer Coaching}

Through engaging in dialogue while working alongside other employees, students employees concurrently learn while doing and interpret interactions to reconstruct knowledge within their own frame of reference. When student employees have the opportunity to interact with more experienced employees, they are introduced to important mental models for solving problems that they can then cognitively test with their prior experiences to develop new knowledge. As they move deeper into their employment experiences, student employees take on the role of formal or informal mentor, guiding newer student employees on the knowledge and skill set needed to 
navigate the job. The element of guiding and teaching adds another layer to their own cognitive development (Wagner \& Gansemer-Topf, 2005).

\section{Observation}

Infusing interpersonal interactions within formal and informal training structures provides additional opportunities for socially constructed learning. Formal training most often occurs at the outset of the employment experience and is primarily used to provide a baseline understanding of how to perform essential job tasks and an acculturation to the workplace (Bauer \& Erdogan, 2011). This workplace activity can be most impactful when student employees are able to experientially participate in learning alongside a more experienced employee, as in shadow training (Fuller \& Unwin, 2011). The learning process should take the form of tell-show-do, in which a more experienced employee explains to a new student employee how to complete a task, has them observe how it is done, and finally has the new employee perform the task, providing guidance and coaching to correct any errors. Interpersonal interactions perhaps play an even more important role in the informal training that takes place throughout employment (Eraut, 2007). Student employees can receive coaching and in-the-moment feedback, delivered by trusted peers and supervisors. Encouraging employees to engage in ongoing dialogue and ask questions ensures that they receive the information they need when it is relevant.

\section{Interactions with Supervisor}

Practitioners can help student employees solidify learning through providing both formal and informal reflective opportunities. Reflection provides student employees with an opportunity to assess experiences and consider future implications (Eraut, 2000).

Though reflection is largely an internal process, supervisors and peers can help facilitate 
reflective processing in student employees by asking probing questions that encourage a deeper level of consideration. Structurally, the workplace environment can also encourage reflection through regularly scheduled check-ins and evaluation periods. Implementation of a localized version of the IOWA GROW® (Guided Reflection on Work) framework, which integrates reflection on learning and transfer of knowledge through a series of brief, structured conversations between supervisors and student employees, is another option for practitioners to consider (Hansen \& Hoag, 2018).

\section{Elevating and Enhancing Environmental Factors}

When structured with solid learning theory in mind, campus workplaces provide myriad opportunities for student employees to engage in learning. During recruitment and hiring, the workplace can start by listing student learning outcomes and departmental mission, vision, and values on application materials (McClellan et al., 2018). This will give applicants an initial opportunity to evaluate if the workplace aligns with their values and future goals. Practitioners need to begin by considering what they are expecting students to learn and develop through campus employment and making learning outcomes explicit at the outset, as early as the recruitment process. There is potential for campus employment to positively affect a number of desired co-curricular and career readiness competencies in undergraduate student employees. Practitioners should review the types of positions available to student employees and consider which outcomes could reasonably be expected to be developed from those opportunities. There are a number of outcomes that practitioners could pull from for this exercise, such as the co-curricular learning outcomes outlined in Learning Reconsidered (Keeling, 2004), NACE career readiness competencies (NACE, 2015), or several others. Student learning outcomes 
should be explicitly stated at the outset of the employment opportunity, revisited throughout, and regularly evaluated (McClellan et al., 2018). The types of activities student employees are exposed to in the campus workplace should also be framed to induce the expected learning outcomes.

\section{Training Methods}

A quality, learning-focused campus workplace environment would provide a number of engagement opportunities from which student employees could learn. These include access to resources, structured and facilitated cross-training and promotional opportunities, reflective activities, and access to increasingly complex instances to engage in problem-solving, among others (Billett, 1996). Though the experience offered by the work environment might be the same for all student employees, each individual employee will engage in it differently depending on individual factors. A student who is apathetic toward their work, for instance, may choose not to seize a promotional opportunity. On the other hand, a highly motivated employee may request to take on additional assignments outside their position description, even if there is no precedence for such a thing within the workplace environment. In this way, the environment both shapes the experience for student employees and is shaped by student employees.

\section{Increasing Responsibility}

Another way in which student employee learning can be supported is through providing access to increasingly challenging activities within the workplace. If student employees are well supported and successful in tackling challenging tasks, their confidence and motivation will increase (Eraut, 2007). Supervisors can support this by continually evaluating student employees' skill set and readiness to take on additional or 
new responsibilities, encouraging them to work just outside their comfort zone, and providing ongoing feedback. Peers, as role models, sounding boards, and coaches can provide social support as students work through challenging projects.

Effective campus employment structures provide facilitated opportunities for student employees to develop a greater depth and breadth of knowledge through their work by establishing formalized promotional and cross-training opportunities. Providing effective and comprehensive initial training, followed up by ongoing training opportunities structured to induce deepening levels of understanding of the work sets the stage for student employees to feel confident in their ability to navigate the responsibilities of the position. This also encompasses setting clear expectations for student employees and holding them accountable to fulfilling the expectations.

\section{Learning Culture}

Grasping an understanding of organizational culture is essential if practitioners are to fully realize the goal of facilitating learning experiences for undergraduate students through campus employment. Tierney (1988) argued that if practitioners hope to implement effective strategies to reach goals, they must be able to recognize the unique structure and components that make up their own organizational culture. Within the campus workplace, such considerations of how student employees feel about the workplace, what connection they have with its mission, and how they interact and share information with others within the workplace can help practitioners understand the culture, and in turn effectively address areas that are incongruent with the desired learning culture.

The environment can support informal training through the establishment of a 
learning culture in which mistakes are considered an essential part of the learning process, rather than something to avoid (Harteis et al., 2008). The environment can cultivate this into the culture through normalizing making mistakes. For instance, supervisors can provide student employees reassurance that there are low consequences for mistakes and share how they have bounced back from mistakes they have made. This may take the form of scaffolding responsibilities so that student employees master lowerlevel responsibilities before proceeding to higher-level responsibilities. This type of culture might also be cultivated through holding student employees accountable for fixing any mistakes and providing the proper support to help them successfully navigate the situation going forward. The workplace might also offer safety nets allowing students to experience the learning benefits of failure without the fear of major repercussions.

Finally, for campus employment to be effectively conceived as a learning opportunity, the environment must be developmental, constructivist, and interculturally inclusive. The learning culture within a learning-centered campus employment experience is first characterized as developmental, providing a quality co-curricular learning experience that is purposeful and intentionally designed to invoke specific learning outcomes. Recognizing that campus employment is just one aspect of the undergraduate experience, it is integrated into the broader mission of the university by engaging students in opportunities to explore areas of passion and apply academic learning experientially.

The second characterization of a learning-centered campus employment experience is constructivist, encouraging student employees to make meaning through active participation and social exchange. Through connecting with others in the campus 
workplace, students are able to externally process and cognitively connect new ideas with previous understandings. By honoring the prior experiences and knowledge that student employees bring into the campus workplace, both collective and individual learning is prioritized.

The third characterization of a learning environment is that it is interculturally inclusive, recognizing that student employees are not a monolithic group, but rather individuals with unique backgrounds and identities. It helps facilitate a sense of belonging, making students feel like valued and accepted members of the community. Students are provided adequate support in their identity-development and are not solely relied on to educate those around them on the history or current challenges of people who share their identity.

\section{Summary of Recommendations}

The Campus Employment Reciprocal Learning Model provides a framework for establishing a campus employment experience that is developmental and educationally purposeful. Practitioners can use the model to effectively structure campus employment as a co-curricular learning experience for undergraduate student employees, supplementing and enhancing the formal academic curriculum. The triadic reciprocal relationships between personal, interpersonal, and organizational factors represent a holistic approach to student engagement, which highlights the institutional responsibility for intentionally structuring campus employment opportunities that are developmental, inclusive, and supportive, while also inviting student employees to be active participants in constructing their learning experience.

Further, the model encourages practitioners to consider the unique composition of 
their campus workplace and evaluate areas in which they can positively leverage influence to encourage learning opportunities. Remembering that the three influential factors interact with each other, practitioners have numerous possibilities for positively influencing change, including making cultural or structural changes to the campus workplace environment, supporting beneficial interpersonal interactions within it, and recognizing, supporting, and cultivating individual strengths. Finally, practitioners can do what is within their control to help student employees experience the benefits of the mediating mechanisms by supporting them in their academic pursuits, allowing them to apply academic learning through employment, demonstrating that they are valued members of the workplace, and helping them develop a greater sense of self-efficacy.

\section{Future Research}

Future research should continue to build our understanding of how campus employment can be effectively structured as a learning opportunity and what student employees learn as a result of their campus employment experiences. The Reciprocal Learning in Campus Employment Model should be empirically tested in specific workplaces to see if it holds in a variety of conditions and position types. This study focused specifically on hourly student employees who worked in student affairs units and another study could focus on other types of student employment experiences, such as contracted workers or residence assistants. A mixed-methods research study might be able to effectively address both aspects. Supervisors of student employees are another group in which further inquiries could be made in understanding their confidence, competence, attitudes, and capacity to enact campus employment as a learning opportunity. 
An additional area of inquiry should include understanding the employment experiences of students from underserved backgrounds. Working college students are not a homogenous group, so understanding the unique opportunities and challenges experienced by various student populations would lead to a better understanding of how campus employment can be realized as a learning opportunity. Focused efforts to understand students who have been underserved by universities and who continue to work more often than their peers would provide insight for practice on how to best support these students in their academic and personal success.

\section{Conclusion}

This study set out to understand the individual, social, and organizational factors that influence learning through campus employment. The research substantiated that campus employment can be effectively facilitated as an educationally purposeful engagement experience for student employees through reciprocal interactions between the three factors. Further, it demonstrated that through campus employment experiences, students are able to develop numerous competencies that complement and extend the academic curriculum. It makes an important contribution to student affairs research in demonstrating how to maximize the potential benefits of campus employment for student employees through the introduction of the Campus Employment Reciprocal Learning Model.

With the wide variety of identities, backgrounds, and experiences of undergraduate student employees and the myriad of campus employment environments, no single formula for transforming campus employment into an educationally purposeful experience exists. However, practitioners who hold responsibility for developing and 
implementing campus employment programs can significantly contribute to the learning potential inherent within that context by recognizing their influence and leveraging opportunities to enhance personal, interpersonal, or environmental factors in facilitating student development of academic and professional identity. 


\section{References}

Argyris, C., \& Schon, D. A. (1978). Organizational learning. Addison-Wesley.

Ashton, D. N. (2004). The impact of organisational structure and practices on learning in the workplace. International Journal of Training and Development, 8(1), 43-53.

Astin, A. W. (1993). What matters in college: Four critical years revisited. Jossey-Bass.

Bauer, T. N., \& Erdogan, B. (2011). Organizational socialization outcomes: Now and into the future. In S. Zedeck (Ed.), APA handbook of industrial and organizational psychology: Maintaining, expanding, and contracting the organization (Vol. 3, pp. 51-64). American Psychological Association.

Baert, S., Marx, I., Neyt, B., Van Belle, E., \& Van Casteren, J. (2018). Student employment and academic performance: An empirical exploration of the primary orientation theory. Applied Economics Letters, 25(8), 547-552.

Bandura, A. (1977). Self-efficacy: toward a unifying theory of behavioral change. Psychological review, 84(2), 191.

Bandura, A. (1986). Social foundations of thought and action: A social cognitive theory. Prentice-Hall, Inc.

Bandura, A. 1997. Self-efficacy: The exercise of control. W. H. Freeman.

Barnett, B., \& Felten, P. (2016). Introduction: Working at the intersections. In B. Barnett \& P. Felten (Eds.) Intersectionality in action: A guide for faculty and campus leaders for creating inclusive classrooms and institutions (pp. xiii-xix). Stylus Publishing, LLC.

Baum, S. (2003). Student work and the financial aid system. In L. W. Perna (Ed.), Understanding the working college student: New research and its implications for 
policy and practice (pp. 3-20). Stylus Publishing, LLC.

Beeson, M. J., \& Wessel, R. D. (2002). The impact of working on campus in the academic persistence of freshmen. Journal of Student Financial Aid, 32(2), 37 45.

Billett, S. (1996). Situated learning: Bridging sociocultural and cognitive theorising. Learning and Instruction, 6(3), 263-280.

Billett, S. (2001). Knowing in practice: Re-conceptualising vocational expertise. Learning and Instruction, 11, 431-452.

Billett, S. (2002). Critiquing workplace learning discourses: Participation and continuity at work. Studies in the Education of Adults, 34(1), 56. https://doi.org/10.1080/02660830.2002.11661461

Billett, S. (2009). Realizing the educational worth of integrating work experiences in higher education. Studies in Higher Education, 34(7), 827-843.

Billett, S. (2014). Mimesis: Learning through everyday activities and interactions at work. Human resource development review, 13(4), 462-482.

Blimling, G. (2015). Student learning in college residence halls: What works, what doesn't, and why. Jossey-Bass.

Broughton, E. A., \& Otto, S. K. (1999). On-campus student employment: Intentional learning outcomes. Journal of College Student Development, 40(1), 87-89.

Burnside, O., Wesley, A., Wesaw, A., \& Parnell, A. (2019). Employing student success: A comprehensive examination of on-campus student employment. Washington, DC: National Association of Student Personnel Administrators.

Cahalan, M., Perna, L. W., Yamashita, M., Ruiz, R., \& Franklin, K. (2017). Indicators of 
higher education equity in the United States: 2017 trend report. Washington, DC: Pell Institute for the Study of Higher Education, Council for Education Opportunity (COE) and Alliance for higher Education and Democracy (AHEAD) of the University of Pennsylvania.

Caldwell, J. (2005). Things fall apart: Discourse on agency and change in organizations. Human Relations, 58(1), 83-114. https://doi.org/10.1177/0018726705050937

Callendar, C. (2008). The impact of term-time employment on higher education students' academic attainment and achievement. Journal of Education Policy, 23(4), 259377.

Carlisle, S. (2015). Learning within collegiate recreation student employment (Publication no. 3723845) [Doctoral dissertation, Northern Illinois University]. Huskie Commons.

Carr, J. W. (2005). An exploration of how learning and development emerge for student employees during the on-campus work experience (Publication no. 3161580) [Doctoral dissertation, The George Washington University]. ProQuest Dissertations Publishing.

Cheng, D. X., \& Alcántara, L. (2007). Assessing working students' college experiences: a grounded theory approach. Assessment \& Evaluation in Higher Education, 32(3), 301-311. https://doi.org/10.1080/02602930600896639

Chickering, A. W., \& Reisser, L. (1993). Education and identity. (2 ${ }^{\text {nd }}$ ed.). Jossey-Bass. Choi, Y. (2018). Student employment and persistence: Evidence of effect heterogeneity of student employment on college dropout. Research in Higher Education, 59(88), 88-107. 
Clarke, N. (2005). Workplace learning environment and its relationship with learning outcomes in healthcare organizations. Human Resource Development International, 8(2), 185-205.

Coe, C., Zehnder, A., \& Kinlaw, D. C. (2008). Coaching for commitment: Coaching Skills Inventory (CSI): Self. Pfeiffer.

College Board. (2017). Undergraduate enrollment and percentage receiving Pell grants over time. Retrieved from https://trends.collegeboard.org/student-aid/figurestables/undergraduate-enrollment-and-percentage-receiving-pell-grants-over-time

Collin, K. (2002). Development engineers' conceptions of learning at work. Studies in Continuing Education, 24(2), 133-152.

Cress, C. M., Astin, H. S., Zimmerman-Oster, K., \& Burkhardt, J. C. (2001). Developmental outcomes of college students' involvement in leadership activities. Journal of College Student Development, 42(1), 15-27.

Cullen, J., Hadjivassiliou, K., Hamilton, E., Kelleher, J., Sommerlad, E., \& Stern, E. (2002). Review of current pedagogic research and practice in the fields of postcompulsory education and lifelong learning. http://www.leeds.ac.uk/educol/documents/00003147.htm

Darolia, R. (2014). Working (and studying) day and night: Heterogenous effects of working on the academic performance of full-time and part-time students. Economics of Education Review, 38, 38-50.

Driver, R., Asoko, H., Leach, J., Scott, P., \& Mortimer, E. (1994). Constructing scientific knowledge in the classroom. Educational researcher, 23(7), 5-12.

Ellinger, A. D. (2005). Contextual factors influencing informal learning in a workplace 
setting: The case of "Reinventing Itself Company". Human Resource Development Quarterly, 16(3), 389-415.

Engeström, Y. (2001). Expansive learning at work: Toward an activity theoretical reconceptualization. Journal of Education and Work, 14(1), 133-156. https://doi.org/10.1080/13639080020028747

Engeström, Y. (2011). Activity theory and learning at work. In M. Malloch, L. Cairns, K. Evans, \& B. N. O’Connor (Eds.), The SAGE handbook of workplace learning (pp. 86-104). SAGE Publications, Inc.

Engle, J., \& Tinto, V. (2008). Moving beyond access: College success for low-income, first-generation students. Pell Institute for the Study of Opportunity in Higher Education. https://eric.ed.gov/?id=ED504448

Eraut, M. (2000). Non-formal learning and tacit knowledge in professional work. British Journal of Educational Psychology, 70(1), 113-136. https://doi.org/10.1348/000709900158001

Eraut, M. (2004). Informal learning in the workplace. Studies in Continuing Education, 26(2), 247-273. https://doi.org/10.1080/158037042000225245

Eraut, M. (2007). Learning from other people in the workplace. Oxford Review of Education, 33(4), 403-422.

Fede, J. H., Gorman, K. S., \& Cimini, M. E. (2018). Student employment as a model for experiential learning. Journal of Experiential Education, 41(1), 107-124.

Fischer, E. M. J. (2007). Settling into campus life: Differences by race/ethnicity in college involvement and outcomes. The Journal of Higher Education, 78(2), 125161. 
Fried, J. (2017). Preface. In J. Fried \& R. Harper (Eds.), Learning everywhere on campus: Teaching strategies for student affairs professionals. Taylor \& Francis.

Fuller, A., \& Unwin, L. (2003). Learning as apprentices in the contemporary UK workplace: Creating and managing expansive and restrictive participation. Journal of Education and Work, 16(4), 407-426.

Fuller, A., \& Unwin, L. (2011). Workplace learning and the organization. In M. Malloch, L. Cairns, K. Evans, \& M. Malloch (Eds.), The SAGE handbook of workplace learning (pp. 46-59). SAGE Publications, Inc.

Furr, S. R., \& Elling, T. W. (2000). The influence of work on college student development. NASPA Journal, 37(2), 454-470.

Gellin, A. (2003). The effect of undergraduate student involvement on critical thinking: A meta-analysis of the literature 1991-2000. Journal of College Student Development, 44(6), 746-762. https://doi.org/10.1353/csd.2003.0066

Hackman, R. J., \& Oldham, G. (1976). Motivation through the design of work: Test of a theory. Organizational Behavior and Human Performance, 16(2), 250-79.

Hager, P. (2011). Theories of workplace learning. In M. Malloch, L. Cairns, K. Evans, \& B. N. O'Connor (Eds.), The SAGE handbook of workplace learning (pp. 17-31). SAGE Publications, Inc.

Hansen, S. L., \& Hoag, B. A. (2018). Promoting learning, career readiness, and leadership in student employment. In S. Komives \& K. Guthrie (Eds.), Leadership development for career readiness in university settings (New Directions for Student Leadership, no. 157, pp. 85-99). Jossey-Bass.

Harteis, C., Bauer, J., \& Gruber, H. (2008). The culture of learning from mistakes: How 
employees handle mistakes in everyday work. International Journal of Educational Research, 47(4), 223-231.

Hochanadel, A., \& Finamore, D. (2015). Fixed and growth mindset in education and how grit helps students persist in the face of adversity. Journal of International Education Research, 11(1), 47-50.

Hodkinson, P., \& Hodkinson, H. (2004). The significance of individuals' dispositions in workplace learning: A case study of two teachers. Journal of Education and Work, 17(2), 167-182.

Hovdhaugen, E. (2015). Working while studying: The impact of term-time employment of dropout rates. Journal of Education and Work, 28(6), 631-651.

Hu, C., \& Kuh, G. D. (2002). Being (dis)engaged in educationally purposeful activities: The influence of student and institutional characteristics. Research in Higher Education, 43(5), 555-575.

Illeris, K. (2011). Workplaces and learning. In M. Malloch, L. Cairns, K. Evans, \& B. N. O'Connor (Eds.), The SAGE handbook of workplace learning (pp. 32-45). SAGE.

Johnson, J. M., \& Rowlands, T. (2012). The interpersonal dynamics of in-depth interviewing. In J. F. Gubrium \& J. A. Holstein (Eds.), The SAGE handbook of interview research: The complexity of the craft (2nd ed., pp. 99-113). SAGE Publications, Inc.

Jones, S. R. (2009). Constructing identities at the intersections: An autoethnographic exploration of multiple dimensions of identity. Journal of college student development, 50(3), 287-304.

Jones, S. R., \& McEwen, M. K. (2000). A conceptual model of multiple dimensions of 
identity. Journal of college student development, 41(4), 405-414.

Kahu, E. R. (2013). Framing student engagement in higher education. Studies in Higher Education, 38(5), 758-773.

Kahu, E. R., \& Nelson, K. (2018). Student engagement in the educational interface: Understanding the mechanisms of student success. Higher Education Research \& Development, 37(1), 58-71.

Kasworm, C. (2010). Adult workers as undergraduates: Significant challenges for higher education policy and practice. In L. W. Perna (Ed.), Understanding the working college student: New research and its implications for policy and practice (pp. 23-42). Stylus Publishing, LLC.

Keeling, R. P. (2004). Learning reconsidered: A campus-wide focus on the student experience. Washington, DC: National Association of Student Personnel Administrators \& American College Personnel Association.

Kim, K., Collins Hagedorn, M., Williamson, J., \& Chapman, C. (2004). Participation in Adult Education and Lifelong Learning: 2000-01 [Data set]. https://doi.org/10.1037/e672832007-012

King, J. E. (2006). Working their way through college: Student employment and its impact on the college experience. Washington, DC: American Council on Education.

Kinzie, J., \& Hurtado, S. (2017). Taking advantage of student engagement results in student affairs. In K. Goodman \& D. Cole (Eds.), Using data-informed decision making to improve student affairs practice (New Directions for Student Services, no. 159 , pp. 35-46). Jossey-Bass. 
Kolb, D. A. (1984). Experiential learning: Experience as the source of learning and development. Prentice-Hall.

Kotzmann, J. (2018). The human rights-based approach to higher education: Why human rights norms should guide higher education law and policy. Oxford University Press.

Kuh, G. D. (1995). The other curriculum: Out-of-class experiences associated with student learning and personal development. The Journal of Higher Education, 66(2), 123-155. https://doi.org/10.1080/00221546.1995.11774770

Kuh, G. D. (2008). High impact educational practices: What they are, who has access to them, and why they matter. Washington, DC: Association of American Colleges \& Universities.

Kuh, G. D. (2009). The National Survey of Student Engagement: Conceptual and empirical foundations. In R. Gonyea \& G. D. Kuh (Eds.), Using NSSE in institutional research (New Directions for Institutional Research, no. 141, pp. 520). Jossey-Bass.

Kuh, G. D., Cruce, T., Shoup, R., Kinzie, J., \& Gonyea, R. (2008). Unmasking the effects of student engagement on first-year college grades and persistence. The Journal of Higher Education, 79(5), 540-563.

Kulm, T. L., \& Cramer, S. (2006). The relationship of student employment to student role, family relationships, social interactions and persistence. College Student Journal, 40(4), 927-938.

Lave, J., \& Wenger, E. (1991). Situated learning: Legitimate peripheral participation. Cambridge University Press. 
Lincoln, Y. S., \& Guba, E. G. (1985). Naturalistic inquiry. SAGE.

Livingstone, D. W. (2001). Expanding notions of work and learning: Profiles of latent power. In T. Fenwick (Ed.) Sociocultural perspectives on learning (New Directions for Adult and Continuing Education, no. 92, pp. 19-30). Jossey-Bass.

Logan, J., Hughes, T., \& Logan, B. (2016). Overworked? An observation of the relationship between student employment and academic performance. Journal of College Student Retention: Research, Theory \& Practice, 18(3), 250-262.

Lundberg, C. A. (2004). Working and learning: The role of involvement for employed students. NASPA Journal (National Association of Student Personnel Administrators, Inc.), 41(2), 201-215. https://doi.org/10.2202/0027-6014.1330

Manuti, A., Pastore, S., Scardigno, A. F., Giancaspro, M. L., \& Morciano, D. (2015). Formal and informal learning in the workplace: A research review. International journal of training and development, 19(1), 1-17.

Marion, R., \& Gonzales, L. D. (2013). Leadership in education: Organizational theory for the practitioner. Waveland press.

Masten, A. S., Best, K. M., \& Garmezy, N. (1990). Resilience and development: Contributions from the study of children who overcome adversity. Development and psychopathology, 2(4), 425-444.

Maxwell, J. A. (2013). Qualitative research design: An interactive approach (3rd ed.). SAGE Publications, Inc.

McClellan, G. S., Creager, K., \& Savoca, M. (2018). A good job: Campus employment as a high-impact practice. Stylus Publishing, LLC.

McCormick, A. C., Moore, J. V., III, \& Kuh, G. D. (2010). Working during college: Its 
relationship to student engagement and educational outcomes. In L. W. Perna (Ed.), Understanding the working college student: New research and its implications for policy and practice (pp. 179-212). Stylus Publishing, LLC.

Merriam, S. B., \& Bierema, L. L. (2014). Adult learning: Linking theory and practice. Jossey-Bass.

Merriam, S. B., \& Tisdell, E. J. (2016). Qualitative research: A guide to design and implementation (4th ed.). Jossey-Bass.

Mounsey, R., Vandehey, M., \& Diekhoff, G. (2013). Working and non-working university students: Anxiety, depression, and grade point average. College Student Journal, 47(2), 379-389.

Museus, S. D., Yi, V., \& Saelua, N. (2016). The impact of culturally engaging campus environments on sense of belonging. The Review of Higher Education, 40(2), 187-215. https://doi.org/10.1353/rhe.2017.0001

National Association of Colleges and Employers. (n.d.). Career readiness defined. https://www.naceweb.org/career-readiness/competencies/career-readiness-defined National Center for Education Statistics. (2016). Digest of education statistics (data set). https://nces.ed.gov/programs/digest/d16/tables/dt16_105.20.asp?current=yes

Nuñez, A. (2009). Latino students' transitions to college: A social and intercultural capital perspective. Harvard Educational Review, 79(1), 22-48,168.

Nunez, A., \& Sansone, V. A. (2016). Earning and learning: Exploring the meaning of work in the experiences of first-generation Latino college students. The Review of Higher Education, 40(1), 91-115.

O'Reilly, C. A., \& Chatman, J. A. (1996). Culture as social control: Corporations, cults, 
and commitment. In B. M. Staw \& L. L. Cummings (Eds.), Research in organizational behavior: An annual series of analytical essays and critical reviews (Vol. 18, pp. 157-200). Elsevier Science/JAI Press.

Pascarella, E. T., \& Terenzini, P. T. (2005). How college affects students: A third decade of research (Vol. 2). Jossey-Bass.

Patton, M. Q. (1990). Qualitative evaluation and research methods (2nd ed.). SAGE. Patton, Michael Q. (2015). Qualitative research and evaluation methods: Integrating theory and practice (4th ed.). SAGE Publications, Inc.

Perna, Laura W. (2003). Introduction. In Understanding the working college student: New research and its implications for policy and practice (pp. xiii-xxvi). Stylus Publishing, LLC.

Perozzi, B. (2009). Enhancing student learning through college employment. Dog Ear.

Pike, G. R., Kuh, G. D., \& Massa-McKinley, R. (2008). First-year students’ employment, engagement, and academic achievement: Untangling the relationship between work and grades. NASPA Journal, 45(4), 560-582.

Pomerantz, N. K. (2006). Student engagement: A new paradigm for student affairs. College Student Affairs Journal, 25(2), 176-185.

Pusser, B. (2010). Of a mind to labor: Reconceptualizing student work and higher education. In L. W. Perna (Ed.), Understanding the working college student: New research and its implications for policy and practice (pp. 134-154). Stylus Publishing, LLC.

Radwin, D., Conzelmann, J. G., Nunery, A., Lacy, T. A., Wu, J., Lew, S., Wine, J., \& Siegel, P. (2018). 2015-16 national postsecondary student aid study (No. NPSAS: 
16 NCES 2018-466). U.S. Department of Education.

Roper, L. D. (2011). Supervising across cultures: Navigating diversity and multiculturalism. New Directions for Student Services, 2011(136), 69-80.

Rutter, M. P., \& Mintz, S. (2016, October 20). The curricular and the co-curricular: The importance of what takes place outside the classroom. Inside Higher Ed. https://www.insidehighered.com/blogs/higher-ed-gamma/curricular-and-cocurricular

Saldaña, J. (2016). The coding manual for qualitative researchers (3rd ed.). SAGE Publications, Inc.

Salisbury, M. H., Pascarella, E. T., Padgett, R. D., \& Blaich, C. (2012). The effects of work on leadership development among first-year college students. Journal of College Student Development, 53(2), 300-324.

Sandelowski, M. (2000). Focus on research methods: Whatever happened to qualitative description? Research in Nursing \& Health, 23, 334-340.

Sanford, N. (1966). Self and society: Social change and individual development. Atherton.

Seidman, I. (2013). Interviewing as qualitative research (4th ed.). Teachers College Press.

Soria, K. M. (2015). Elevating the academic success of working-class college students through high-impact educational practices. In D. Mitchell Jr., K. M. Soria, E. A. Daniele, \& J. A. Gipson (Eds.), Student involvement and academic outcomes: Implications for diverse college student populations (pp. 41-56). Peter Lang Publishing, Inc. 
Stasz, C. (2001). Assessing skills for work: Two perspectives. Oxford Economic Papers, 53(3), 385-405.

Stock-Ward, S. R., \& Javorek, M. E. (2003). Applying theory to practice: Supervision in student affairs. NASPA Journal, 40(3), 77-92.

Strauss, L. C., \& Terenzini, P. T. (2007). The effects of students' in- and out-of-class experiences on their analytical and group skills: A study of engineering education. Research in Higher Education, 48(8), 967-992. https://doi.org/10.1007/s11162007-9057-4

Strayhorn, T. (2012). College students' sense of belonging: A key to educational success for all students. Routledge.

Student Employment Enhancement. (n.d.). Retrieved May 6, 2019, from Home website: https://uosee.uoregon.edu/

Suskie, L. (2015). Introduction to measuring co-curricular learning. In L. KennedyPhillips, A. Baldasare, \& M. Christakis (Eds.), Measuring cocurricular learning: The role of the IR office (New Directions for Institutional Research, no. 164, pp. 5-14). Jossey-Bass.

Tierney, W. G. (1987). Facts and constructs: Defining reality in higher education organizations. Review of Higher Education, 11(1), 61-73.

Tierney, W. G. (1988). Organizational culture in higher education: Defining the essentials. The Journal of Higher Education, 59(1), 2-21.

Tuttle, T., McKinney, J., \& Rago, M. (2005). College students working: The choice nexus. (pp. 1-11). Indiana Project on Academic Success.

Wagner, M., \& Gansemer-Topf, A. (2005). Learning by teaching others: A qualitative 
study exploring the benefits of peer teaching. Landscape journal, 24(2), 198-208.

Watkins, K., \& Marsick, V. (1992). Towards a theory of informal and incidental learning in organizations. International Journal of Lifelong Education, 11(4), 287-300.

Weiss, R. S. (1994). Learning from strangers: The art and method of qualitative interview studies. The Free Press.

Wenger, E. (2000). Communities of practice and social learning systems. Organization, $7(2), 225-246$.

Wexley, K. N., \& Latham, G. P. (2000). Developing and training human resources in organizations (Vol. 3). Prentice-Hall.

Winkler, I. (2009). Term-time employment: Exploring the influence of self-identity, motivation and social issues. Education + Training, 51(2), 124-138. https://doi.org/10.1108/00400910910941282 


\section{Appendix A}

\section{Email to Supervisors}

Dear [Student Supervisor Name],

I am writing to ask your assistance in identifying current eligible student employees who may be interested in participating in my doctoral research project titled Where's the Learning in On-Campus Student Employment? An Inquiry into the Experiences of Undergraduate Student Employees. This study will explore how undergraduate student employees' work experiences facilitate learning in the campus employment environment.

Previous research on student employment paints a complicated picture. While much of the literature pertaining to on-campus employment points to its potential to provide a structured, experiential learning opportunity in a way that supports student learning and growth, there are still questions about how that learning can be most effectively supported.

The purpose of this study is to explore student employees' experiences in campus employment within student affairs units to gain a deeper understanding of the individual, social, and organizational factors that influence learning in the campus employment environment. Selected participants will be asked to participate in a 90-minute interview with me. Research participation will be kept confidential and the participants' identities will be only known by me. Student employees who participate in this project will provide insights into how to most effectively structure campus employment experiences to holistically contribute to student learning.

I am asking your help in identifying students who meet the following criteria:

1. Current full-time undergraduate student at this university

2. Currently employed by a unit within the [division of student affairs]

3. Have been employed on campus for at least three terms

I would appreciate you putting forth any student employees who you supervise or work with in your department that meet the criteria by replying to this email with their name(s) and email address(es) by [date]. I will follow up with all student employees put forth to gauge their interest in participating. Due to study size limitations, it is possible that not all student employees identified will be able to participate in the study.

If you have any questions, please reach out to me at trishd@uoregon.edu or 541-3461043. I appreciate your consideration in assisting me with this outreach request.

Sincerely, Trish Dorman 
Appendix B

Email to Potential Participants

Dear [Student Name],

My name is Trish Dorman and I am a current doctoral student at Portland State

University as well as the Associate Director for Business Operations at the University of

Oregon Physical Education and Recreation Department. I am writing to ask you to consider participating in my doctoral research study titled Where's the Learning in OnCampus Student Employment? An Inquiry into the Experiences of Undergraduate Student Employees. I am interested in exploring how undergraduate student employees' work experiences facilitate learning in the campus employment environment. Previously I reached out to student supervisors to ask for referrals of student employees who met the study criteria and might be interested in participating and you were referred by [name].

If selected to participate in this study, you will be asked to participate in a 90-minute interview with me. Your participation in the study will be kept confidential and will not affect your work or be reported back to your supervisor. A pseudonym will be used in place of your name in all interview transcriptions, researcher memos, data analysis, the final report, and any subsequent papers or presentations.

If selected to participate in this study, you will provide valuable insight into how to most effectively structure campus employment experiences to holistically contribute to student learning. Additionally, individuals selected to participate will receive a \$20 [campus bookstore name] gift card.

The following criteria must be met to participate in the study:

1. Current full-time undergraduate student at this university

2. Currently employed by a unit within the [division of student affairs]

3. Have been employed on campus for at least three terms

The perspectives and experiences you bring as a student employee are invaluable to this study. If you are willing and available to participate in the study, please fill out this form [insert Qualtrics link]. Due to study size limitations, it is possible that not all interested student employees will be selected to participate in the study.

If you have any questions, please reach out to me at [email address] or [phone number]. I appreciate your consideration in assisting me with this outreach request.

Sincerely,

Trish Dorman 
Appendix C

Qualtrics Form

Full Name: (Open Response)

Email Address: (Open Response)

Are you a current full time undergraduate student? (Yes/No)

Which Division of Student Life department do you currently work in: (Select One) Dean of Students, EMU (Student Union), Physical Education \& Recreation, University

Career Center

How long have you been employed by this department? (Select One; if 1 or 2 terms is selected, add logic to end survey)

$1-15+$ terms

What is your current job title? (Open Response)

Briefly describe your job duties: (Open Response)

Have you worked in any other positions in this department? (Yes/No)

If yes, please list the job title(s) of any other positions you have held within this department.

During a typical week, how many hours do you work at this job? (Open Response)

Do you hold any other jobs currently? (Yes/No; add logic to describe if yes)

If yes, please describe your other job commitments.

Did you work prior to coming to college? (Yes/No)

Gender: (Open Response)

Age: (Open Response)

Race: (Open Response)

Ethnicity: (Open Response) 
Year in College: (Select One)

1st, 2nd, 3rd, 4th, 5th+ 
Appendix D

Informed Consent Form

\section{Consent to Participate in Research}

Project Title: Where's the Learning in On-Campus Student Employment? An Inquiry into the Experiences of Undergraduate Student Employees

Researcher: Patricia Dorman, Doctoral Candidate in the College of Education Portland State University

Researcher Contact: [email]/[phone number]

You are being asked to take part in a research study. The box below highlights key information about this research for you to consider when making a decision whether or not to participate. Carefully review the information provided on this form. Please ask questions about any of the information you do not understand before you decide to participate.

\section{Key Information for You to Consider}

- Voluntary Consent. You are being asked to volunteer for a research study. It is up to you whether you choose to participate or not. There is no penalty if you choose not to participate or discontinue participation.

- Purpose. The purpose of this research is to explore how undergraduate student employees' work experiences facilitate learning in the campus employment environment.

- Duration. It is expected that your participation will last a total of 2 hours.

- Procedures and Activities. You will be asked to participate in one 90-minute interview with the researcher to share your campus employment experiences.

- Risks. Although risks are minimal, you may experience discomfort when responding to questions asked during the interview as you reflect on specific employment experiences and share your perspective.

- Benefits. No direct benefit, but your participation may help inform improved campus employment experiences for future student employees.

- Alternatives. Participation is voluntary and the only alternative is to not participate.

\section{Why is this research being done?}

The purpose of this study is to explore student employee experiences in campus employment at a large, public Pacific Northwest university to gain a deeper 
understanding of the individual, social, and organizational factors that influence learning in the campus employment environment. You are being asked to participate because you are a currently enrolled undergraduate student who has been employed by a department within student affairs for at least three terms. About 10-12 people will take part in this research.

\section{How long will $I$ be in this research?}

I expect that your participation will last for one 90-minute interview with me, the researcher.

\section{What happens if I agree to participate?}

If you agree to be in this research, your participation will include answering questions about your student employment experience during an interview. Interviews will be audio recorded and I will be taking notes. I will tell you about any new information that may affect your willingness to continue participation in this research.

\section{What happens to the information collected?}

Information collected for this research will be used to inform my doctoral dissertation and any subsequent related journal articles and presentations. Identifiable information will not be included in any published work or presentations.

\section{How will my privacy and data confidentiality be protected?}

I will take measures to protect your privacy including protecting your identity and maintaining confidentiality. Despite taking steps to protect your privacy, we can never fully guarantee that your privacy will be protected.

To protect the security of all of your personal information, I will assign you a pseudonym at the outset of the study that will be used throughout data analysis and in the final report. Additionally, audio recordings, transcriptions, and researcher memos will be kept in a password-protected file on the researcher's computer. Despite these precautions, we can never fully guarantee the confidentiality of all study information.

Individuals and organizations that conduct or monitor this research may be permitted access to inspect research records. This may include private information. These individuals and organizations include the Institutional Review Board that reviewed this research and the researcher's dissertation chair.

\section{What are the risks if I participate?}

Although risks are minimal, you may experience discomfort when responding to questions asked during the interview as you reflect on specific employment experiences 
and share your perspective. You are encouraged to ask questions or raise concerns at any time about the nature of the study or the methods I am using.

\section{What are the benefits if I participate?}

You may benefit from participating in this research through spending time reflecting on your employment experience and engaging in interesting and thought-provoking discussion. Additionally, your participation may help inform improved campus employment experiences for future student employees.

\section{What if I want to stop participating in this research?}

Your participation is voluntary. You do not have to take part in this study, but if you do, you may stop at any time. You have the right to choose not to participate in any study activity or completely withdraw from participation at any point without penalty or loss of benefits to which you are otherwise entitled. Your decision whether or not to participate will not affect your relationship with the researcher or Portland State University.

\section{Will I be paid for participating in this research?}

Upon completion of the interview, you will receive a \$20 [book store] gift card.

\section{Who can answer my questions about this research?}

If you have questions, concerns, or have experienced a research related injury, contact the researcher at:

Patricia Dorman

[phone number]

[email address]

\section{Who can I speak to about my rights as a research participant?}

The Portland State University Institutional Review Board ("IRB") is overseeing this research. The IRB is a group of people who independently review research studies to ensure the rights and welfare of participants are protected. The Office of Research Integrity is the office at Portland State University that supports the IRB. If you have questions about your rights, or wish to speak with someone other than the research team, you may contact:

Office of Research Integrity

PO Box 751

Portland, OR 97207-0751

Phone: (503) 725-5484

Toll Free: 1 (877) 480-4400

Email: hsrrc@pdx.edu 


\section{Consent Statement}

I have had the opportunity to read and consider the information in this form. I have asked any questions necessary to make a decision about my participation. I understand that I can ask additional questions throughout my participation.

By signing below, I understand that I am volunteering to participate in this research. I understand that I am not waiving any legal rights. I have been provided with a copy of this consent form. I understand that if my ability to consent for myself changes, either I or my legal representative may be asked to provide consent prior to me continuing in the study.

I consent to participate in this study.

\section{Name of Adult Participant Signature of Adult Participant Date}

Researcher Signature (to be completed at time of informed consent)

I have explained the research to the participant and answered all of his/her questions. I believe that he/she understands the information described in this consent form and freely consents to participate.

\section{Name of Research Team Member Signature of Research Team Member Date}


Appendix E

Interview Protocol

\begin{tabular}{|c|c|c|}
\hline Question(s) & Purpose & $\begin{array}{l}\text { Related } \\
\text { Theory/Construct }\end{array}$ \\
\hline $\begin{array}{l}\text { Tell me about your work with } \\
\text { [campus department]. }\end{array}$ & $\begin{array}{l}\text { Allow student employee to describe } \\
\text { their work in their own words } \\
\text { without priming the learning } \\
\text { construct. }\end{array}$ & N/A \\
\hline $\begin{array}{l}\text { What made you select this } \\
\text { position? } \\
\text { - What does this job } \\
\text { mean to you? }\end{array}$ & $\begin{array}{l}\text { Understand individual motivations } \\
\text { for engaging in work }\end{array}$ & $\begin{array}{l}\text { Individual } \\
\text { epistemologies of } \\
\text { learners }\end{array}$ \\
\hline $\begin{array}{l}\text { What are your reasons for } \\
\text { working while attending } \\
\text { college? }\end{array}$ & $\begin{array}{l}\text { Understand individual motivations } \\
\text { for engaging in work }\end{array}$ & $\begin{array}{l}\text { Individual } \\
\text { epistemologies of } \\
\text { learners }\end{array}$ \\
\hline $\begin{array}{l}\text { Describe in detail your job } \\
\text { responsibilities in your } \\
\text { current position. } \\
\text { - Walk me through } \\
\text { what a typical shift } \\
\text { looks like. } \\
\text { - How did you learn to } \\
\text { do those things? } \\
\text { - How have your } \\
\text { responsibilities } \\
\text { changed over time? }\end{array}$ & $\begin{array}{l}\text { Understand the organizational } \\
\text { context of the student position }\end{array}$ & $\begin{array}{l}\text { Sociocultural } \\
\text { Workplace Learning } \\
\text { Theories }\end{array}$ \\
\hline $\begin{array}{l}\text { How would you describe the } \\
\text { knowledge and skill set a } \\
\text { person would need to } \\
\text { successfully do your job? } \\
\text { - How have you } \\
\text { personally developed } \\
\text { these skills? }\end{array}$ & $\begin{array}{l}\text { Understand how employees } \\
\text { conceptualize learning and their } \\
\text { perceptions of skill development }\end{array}$ & $\begin{array}{l}\text { Sociocultural } \\
\text { Workplace Learning } \\
\text { Theories }\end{array}$ \\
\hline $\begin{array}{l}\text { In which ways have your } \\
\text { personal background and } \\
\text { previous educational }\end{array}$ & $\begin{array}{l}\text { Understand how personal } \\
\text { backgrounds and prior educational } \\
\text { experiences influence current work }\end{array}$ & $\begin{array}{l}\text { Individual } \\
\text { epistemologies of } \\
\text { learners }\end{array}$ \\
\hline
\end{tabular}




\begin{tabular}{|c|c|c|}
\hline $\begin{array}{l}\text { experiences helped prepare } \\
\text { you to perform your job } \\
\text { duties in this position? }\end{array}$ & & \\
\hline $\begin{array}{l}\text { What did you expect to get } \\
\text { out of your job when you } \\
\text { started working here? } \\
\text { - How has your actual } \\
\text { work aligned with } \\
\text { your expectations? }\end{array}$ & $\begin{array}{l}\text { Understand alignment between } \\
\text { individual interest and perceived } \\
\text { value of work }\end{array}$ & $\begin{array}{l}\text { Individual } \\
\text { epistemologies of } \\
\text { learners }\end{array}$ \\
\hline $\begin{array}{l}\text { In which ways do you work } \\
\text { with other people in your job? } \\
\text { - What, if anything, } \\
\text { have you learned } \\
\text { through these } \\
\text { interactions? }\end{array}$ & $\begin{array}{l}\text { Understand the social dynamics at } \\
\text { work and how the worker describes } \\
\text { their influence on learning. }\end{array}$ & Student Engagement \\
\hline $\begin{array}{l}\text { What are some things that } \\
\text { you have learned through } \\
\text { working in this job? } \\
\text { - Who or what } \\
\text { specifically } \\
\text { contributed to that } \\
\text { learning? } \\
\text { - In which ways do you } \\
\text { think your workplace } \\
\text { supports your } \\
\text { learning? }\end{array}$ & $\begin{array}{l}\text { Understand what has been learned } \\
\text { and to what or whom the student } \\
\text { attributes the learning }\end{array}$ & $\begin{array}{l}\text { Sociocultural } \\
\text { Workplace Learning } \\
\text { Theories }\end{array}$ \\
\hline $\begin{array}{l}\text { When you think about what } \\
\text { you have learned in your } \\
\text { academic classes, in which } \\
\text { ways have you been able to } \\
\text { apply that knowledge into } \\
\text { your work? } \\
\text { Tell me about a time } \\
\text { that you applied } \\
\text { something you learned } \\
\text { in class at work. }\end{array}$ & $\begin{array}{l}\text { Understand the ways in which } \\
\text { learning is extended beyond the } \\
\text { classroom }\end{array}$ & Student Engagement \\
\hline $\begin{array}{l}\text { Describe a time that you } \\
\text { encountered a situation at } \\
\text { work that you didn't know } \\
\text { how to handle. }\end{array}$ & $\begin{array}{l}\text { Understand how new knowledge and } \\
\text { skills are acquired and synthesized } \\
\text { into practice through active problem- } \\
\text { solving. }\end{array}$ & $\begin{array}{l}\text { Sociocultural } \\
\text { Workplace Learning } \\
\text { Theories }\end{array}$ \\
\hline
\end{tabular}




\begin{tabular}{|c|c|c|}
\hline $\begin{array}{l}\text { What resources did } \\
\text { you use to navigate } \\
\text { the situation? }\end{array}$ & & \\
\hline $\begin{array}{l}\text { When you reflect back upon } \\
\text { the beginning of your } \\
\text { employment to now, what are } \\
\text { some of the ways in which } \\
\text { you have changed or } \\
\text { developed? } \\
\text { - To what or whom do } \\
\text { you attribute those } \\
\text { changes? }\end{array}$ & $\begin{array}{l}\text { Understand how students have } \\
\text { changed through their work and what } \\
\text { accounts for that change }\end{array}$ & Student Engagement \\
\hline $\begin{array}{l}\text { How do you see the skills and } \\
\text { knowledge that you have } \\
\text { gained in this job connecting } \\
\text { to future job opportunities? }\end{array}$ & $\begin{array}{l}\text { Understand the relevance and } \\
\text { saliency of current workplace } \\
\text { learning to future skills }\end{array}$ & Student Engagement \\
\hline
\end{tabular}

